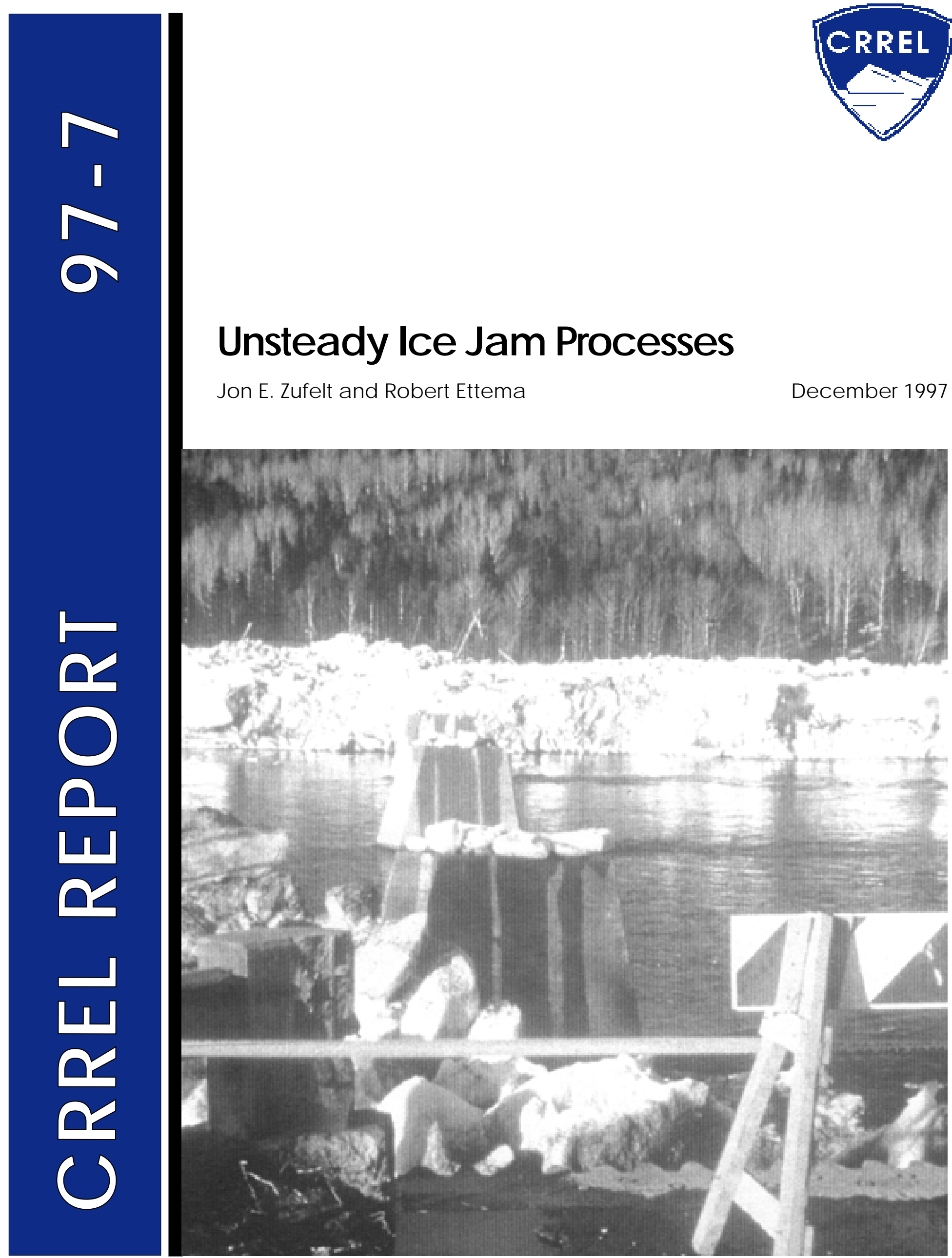


Abstract: Ice jams cause flooding in northern temperate-climate areas, usually forming rapidly, often with little waming, constricting water flow and elevating water levels. Consequently, jam formation comprises highly unsteady processes: drifting ice pieces are brought to rest, accumulated ice shoves and thickens, and initial water depths and velocities change. Those processes are even more unsteady when a jam collapses. Prior simulations of ice jams, however, treat them as simply stationary, uniformly thick accumulations of ice pieces. No account is taken of the impact forces exerted by moving ice, an estimation that is further complicated by the need to couple equations describing water flow and ice movement. Under the dynamic conditions at- tendant to jam formation, water flow and ice movement interactively influence each other. This report evaluates the importance of ice momentum on ice jam thickness and thickness distribution using experiments conducted with laboratory flumes and a numerical model in which the equations of motion for one-dimensional flow of water and ice are solved as fully coupled. In this regard, the model is unique, enabling simulation of the important unsteady interactions of water and ice, and determination of their effects on jam thickness. Ice momentum should be taken into account for most jams because it leads to significantly thicker jams and affects the thickness profile. A useful dimensionless parameter is identified for generalizing this finding.

Cover: Example of destructive power of a highly dynamic ice jam on the St. J ohn River.

\section{How to get copies of CRREL technical publications:}

Department of Defense personnel and contractors may order reports through the Defense Technical Information Center:

DTIC-BR SUITE 0944

8725 J OHN J KINGMAN RD

FT BELVOIR VA 22060-6218

Telephone 18002253842

E-mail help@dtic.mil

msorders@dtic.mil

WWW http://www.dtic.dla.mil/

All others may order reports through the National Technical Information Senvice:

NTIS

5285 PORT ROYAL RD

SPRINGFIELD VA 22161

Telephone 17034874650

17034874639 (TDD for the hearing-impaired)

E-mail_orders@ntis.fedworld.gov

WWW http://www.fedworld.gov/ntis/ntishome.html

A complete list of all CRREL technical publications is available from: USACRREL (CECRL-LP)

72 LYME RD

HANOVER NH 03755-1290

Telephone 16036464338

E-mail techpubs@crrel.usace.army.mil

For information on all aspects of the Cold Regions Research and Engineering Laboratory, visit our World Wide Web site: http://www.crrel.usace.army.mil 


\section{CRREL Report 97-7}

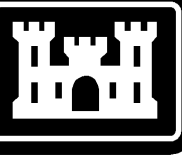

US Army Corps of Engineers $₫$

Cold Regions Research \& Engineering Laboratory

\section{Unsteady Ice Jam Processes}

Jon E. Zufelt and Robert Ettema 


\section{PREFACE}

This report was prepared by Dr. Jon E. Zufelt, Research Hydraulic Engineer, Ice Engineering Research Division, Research and Engineering Directorate, U.S. Army Cold Regions Research and Engineering Laboratory, and Dr. Robert Ettema, Professor and Research Engineer, The University of Iowa, Iowa Institute of Hydraulic Research (IIHR). The work was funded by the Office of the Chief of Engineers through CWIS, Work Unit, Model Studies of Ice Jams.

The authors thank Dr. Jean-Claude Tatinclaux, Chief, Ice Engineering Research Division, and Dr. George Ashton, Chief, Research and Engineering Directorate, CRREL, for their technical reviews of this report.

The contents of this report are not to be used for advertising or promotional purposes. Citation of brand names does not constitute an official endorsement or approval of the use of such commercial products. 


\section{CONTENTS}

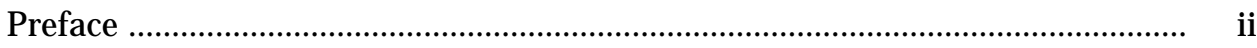

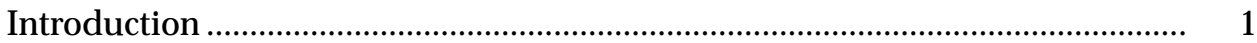

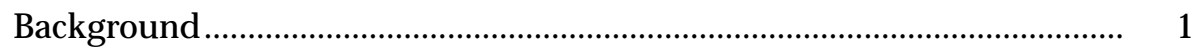

Need for research ......................................................................................... 1

Field observations ..................................................................................... 2

Objective and approach .......................................................................... 4

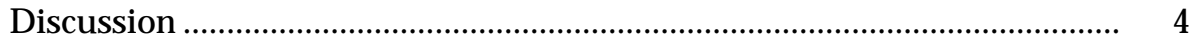

Review of ice jam modeling .............................................................................. 5

Review of ice jam classification ............................................................ 5

Analysis of stationary jams ..................................................................... 11

Numerical modeling ........................................................................ 17

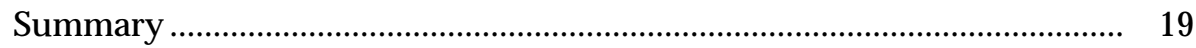

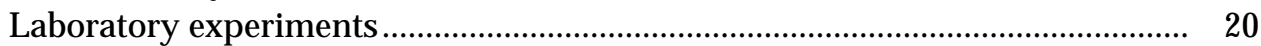

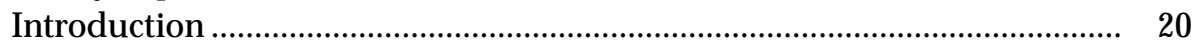

Experimental setup .......................................................................... 21

Observations of shoving and thickening ………........................................ 23

Equilibrium thickness evaluations ............................................................ 25

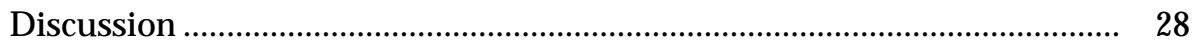

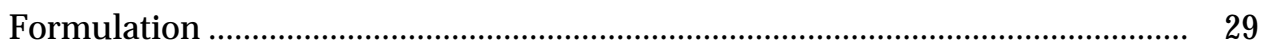

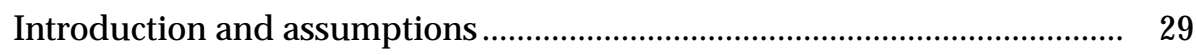

Development of equations ...................................................................... $\quad 30$

Discretization of the system of equations .................................................. 42

Solution of the system of equations ........................................................ 47

Ice cover stability, solution methods, and boundary conditions ................. 49

Numerical model description ......................................................................... 55

Model description and capabilities ....................................................... 55

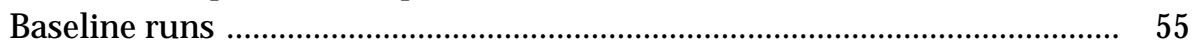

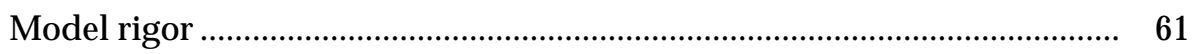

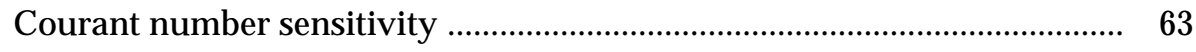

Theta-weighting factor analysis ................................................................ 63

Alternate boundary conditions ................................................................. 65

Effects of variable length steps ............................................................... 66

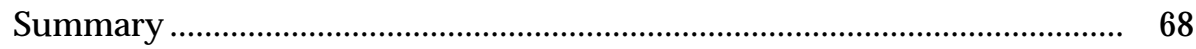

Unsteady jam dynamics .......................................................................... 68

Effects of ice momentum ......................................................................... 68

Comparison with steady-state models ..................................................... $\quad 70$

Dimensionless momentum parameter ..................................................... $\quad 72$

Effects of hydrograph shape on jam thickening ......................................... 75

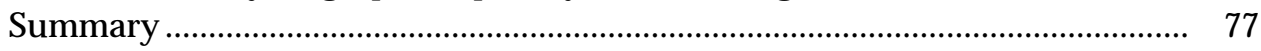

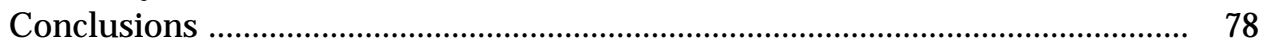

Recommendations for future research .......................................................... 79

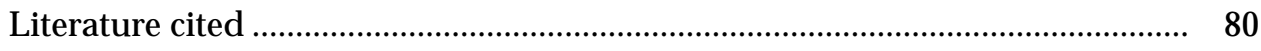

Appendix A …...................................................................................... 83

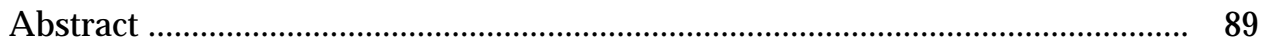


Figure

1. Ice jam at first signs of failure ……………………....................................... 2

2. Ice jam failure at beginning of ice motion ........................................................ 3

3. Ice jam failure with ice fully mobilized ……………….................................... 3

4. River channel following ice jam failure ........................................................... 4

5. Cross section of a freezeup jam …………................................................... 6

6. Surface jam resulting from juxtaposition of frazil pans .................................. 6

7. Freezeup jam following shoving and thickening ............................................ 7

8. Temporal variation of air temperature and location of upstream edge of a freezeup jam on the Salmon River, Idaho ................................................ 8

9. Cross section of a breakup jam .................................................................. 8

10. Mid-winter breakup jam on the Kennebec River in Maine ............................ 9

11. Stage record for USGS station no. 0101000 on the St. John River in Maine during 1994 breakup ................................................................... $\quad 10$

12. Idealization of ice jam evolution with time ….............................................. 12

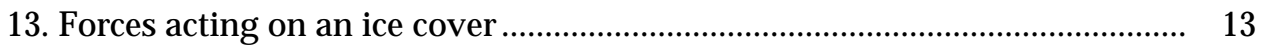

14. Dimensionless stability parameter …………............................................. 15

15. CRREL refrigerated, tiltable flume ............................................................ 21

16. Plastic beads used to simulate ice ………….............................................. 22

17. Progressive jam failure .............................................................................. 23

18. Measured velocity profile with fitted log-law equations for the ice

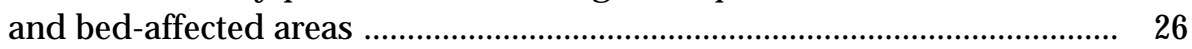

19. Slope vs. discharge, showing increase in slope after jam failure .................. 27

20. Calculated equilibrium jam thickness vs. discharge .................................... 27

21. Change in jam thickness with step increases in discharge .......................... 28

22. Measured vs. predicted jam thickness following progressive and complete jam failures ........................................................................ 28

23. Ice and water flows .................................................................................. 29

24. Longitudinal and cross-sectional views of ice and water flow areas, showing coordinate system used in equation development ....................... 31

25. Forces acting on the water control volume .................................................... 32

26. Two-layer approach designation of shear stress due to water flow ............. 34

27. Shear stress due to water flow for cases of moving jam .............................. 35

28. Shear force on the ice jam underside vs. ice velocity ..................................... 36

29. Forces acting on the ice control volume ……............................................... 37

30. Computational grid used for the numerical simulations ............................ 44

31. Coefficient matrix for the Newton-Raphson iteration procedure ................ 49

32. Conditions of ice jam stability for a computational reach ............................ 51

33. Block diagram for the fully coupled solution scheme.................................. 52

34. Block diagram for the loosely coupled solution scheme ............................... 53

35. Upstream water discharge hydrograph for baseline run ............................. 56

36. Output plots of solution variables for baseline run ........................................ 58

37. Water depth and velocity profiles at various times for the initial
condition of local depth increase .............................................................

38. Initial and final water surface level profiles for depth increase ................... 61

39. Water depth and velocity profiles at various times for the initial condition of local depth increase ............................................................... 62

40. Initial and final water surface level profiles for depth decrease ................... 62 
41. Initial and final water surface level and bottom of ice profiles for an ice jam having nonuniform thickness ............................................... 63

42. Final jam thickness profiles for two initially unstable jam thicknesses ....... 63

43. Final jam thickness profiles for Courant numbers of 1, 3, 5, 10, 12, and 24 . 64

44. Final jam thickness profiles for $\theta_{i}=1.0$ and $\theta=0.55,0.6,0.66,0.8$, and 1.0 . 64

45. Final jam thickness profiles for $\theta=0.6$ and $\theta_{i}=0.55,0.6,0.66,0.8$, and 1.0 . 65

46. Final bed, bottom of jam, and water surface level profiles for the condition of downstream depth being held at $3.0 \mathrm{~m}$

47. Final bed, bottom of jam, and water surface level profiles for the condition of downstream depth being held at an initial depth of $1.729 \mathrm{~m}$

48. Final jam thickness profiles for upstream jam thickness being held at $1.47,1.4$, and $1.3 \mathrm{~m}$

49. Final jam thickness profile for length step reduced to $25 \mathrm{~m}$

50. Final jam thickness profile for length step changes in the first $2000 \mathrm{~m}$ of the system

51. Final jam thickness profile for random length steps throughout the system

52. Comparison of thickness profile predicted by static-unsteady thickness model to fully coupled model and equilibrium thickness

53. Comparison of thickness profile predicted by loosely coupled model to fully coupled model....

54. Discharge record during breakup jam initiation and failure ......

55. Hypothetical breakup jam discharge hydrograph....

56. Final jam thickness profiles for two steady water discharges compared to the fully coupled model results 3

57. Water discharge at upstream end, mid-reach, and downstream end of the system.

58. Dimensionless jam thickness vs. ice momentum parameter for several bed slopes 70

71

72

59. Final jam thickness profiles for large, medium, and small ice momentum effects

60. Inflow hydrographs for various times to peak …………............................. 76

61. Dimensionless jam thickness vs. $t_{\mathrm{p}} / t_{\mathrm{b}}$ at three bed slopes ........................... 76

62. Effect of $t_{\mathrm{p}}$ on final jam thickness profile shape ............................................ 76

63. Inflow hydrographs for various times of sustained flow ............................. 77

64. Dimensionless jam thickness vs. $t_{\mathrm{s}} / t_{\mathrm{b}}$ at three bed slopes ........................... 77

65. Effect of $t_{\mathrm{s}}$ on final jam thickness profile shape ............................................. 77

\section{TABLES}

Table

1. List of baseline testing parameters ..................................................................... 56

2. Ice parameters for channels at different bed slopes ...................................... 73

3. Characteristics of various inflow hydrographs .............................................. 74 


\title{
Unsteady Ice Jam Processes
}

\author{
JON E. ZUFELT AND ROBERT ETTEMA
}

\section{INTRODUCTION}

\section{Background}

Ice jams cause massive damage annually throughout the world's northern temperate regions. In the U.S. alone, the annual damages from ice jams average \$125 million (USACE 1994). These include property losses, emergency assistance, flood insurance, and increased operation and maintenance costs, replacement of infrastructure, and loss of hydropower revenues. Most damages are caused by high water levels associated with ice jams, though some are from the direct impact of moving ice during ice runs.

The formation and evolution of ice jams comprise a series of inherently unsteady processes, in which moving ice is brought to rest in accumulations that shove and thicken in accordance with changing forces exerted by water flow, accumulation weight, and bank roughness. These processes are even more unsteady when a jam collapses, plows downstream, and possibly reforms. Prior formulations for ice jams treat them as stationary, steady-state ice accumulations that are subject to invariant flow conditions. This study, however, presents the first formulation for and examination of the fully coupled dynamic nature of the unsteady processes associated with jam formation.

\section{Need for research}

In efforts to protect life and property from the damages attendant to ice jams and related flooding, prior models were developed to predict water-level changes caused by ice jams. Those models treat the evolution of ice jam thickness (shoving and thickening) as quasi-steady, with jam thickness spontaneously adjusting to a new equilibrium value in concert with water flow changes. Steady-flow models, such as HEC-2 modified with ice cover option, simply provide the steady water levels that would exist with a uniformly thick jam already in place. The long-standing assumption used is of an ice jam of equilibrium thickness, floating in static force equilibrium just on the verge of stability or failure. Other models simulate the unsteadiness of the water flow using the conservation of mass and momentum equations for the water, but solve in an uncoupled manner for thickness between time steps, again by the static force balance.

There currently are no formulations that describe the coupled interaction of the water and ice movement and their effects on flow depth and ice thickness. Also, no information exists on how jams evolve, fail, and thicken. In that regard, the following important groups of questions need to be addressed:

- How do ice jams evolve? Present formulations allow for instantaneous changes in the jam thickness attributable to changes in the forces acting on the jam. No account is currently made for the impact forces generated by moving ice. Once a jam fails, ice is mobilized and travels downstream, often at high speed (Henderson and Gerard 1981). Do jams move and then thicken upon failure, thicken as they fail, or thicken and result in a progressive downstream-moving failure? 
- To what extent does ice momentum affect jam thickness? If jams move upon failure, the force levels acting on the jam inevitably change. What are these changes and how do they affect the forces acting on the jam?

- What are the effects of the interaction of the water and ice motion? The water shear stress on the jam underside is one of the principal forces on the jam. When a jam fails and moves, however, this force is reduced, which interactively reduces the resistance to water flow. As water and ice motion are interrelated, what are the consequences, for jam thickness prediction, of uncoupling their influences as is currently done in existing formulations?

\section{Field observations}

The inherent unsteadiness of jam formation and failure is obvious from field observations. As an example, observations of a freezeup jam on the Salmon River near Salmon, Idaho, made from a small bridge about $1 \mathrm{~km}$ downstream of the leading edge (head) of the jam, are presented here. The jam was approximately $25 \mathrm{~km}$ long, and, owing to mild weather $\left(-3^{\circ} \mathrm{C}\right)$, the leading edge had been stationary over the night. Water levels were slightly more than bankfull at the bridge and thick frazil accumulations filled the channel.

The water level began rising, first noticeably at the treeline along the bank, then in mid-channel, as the water began to flood the surface of the jam (Fig. 1). The ice in the channel appeared to rise slightly, and shear cracks could be seen forming about $10 \mathrm{~m}$ out from the banks. The water levels continued to rise and the ice, groaning, began to slowly move downstream en masse (Fig. 2). In a matter of seconds, the entire channel section of the jam for about $1 \mathrm{~km}$ on either side of the bridge (between the shear cracks) was moving downstream (Fig. 3). As the ice moved, the water level fell, until the center portion of the channel was clear of ice upstream to where the leading edge had previously been located. Once the ice had passed, water levels dropped by approximately $0.5 \mathrm{~m}$, exposing shear walls of ice along the

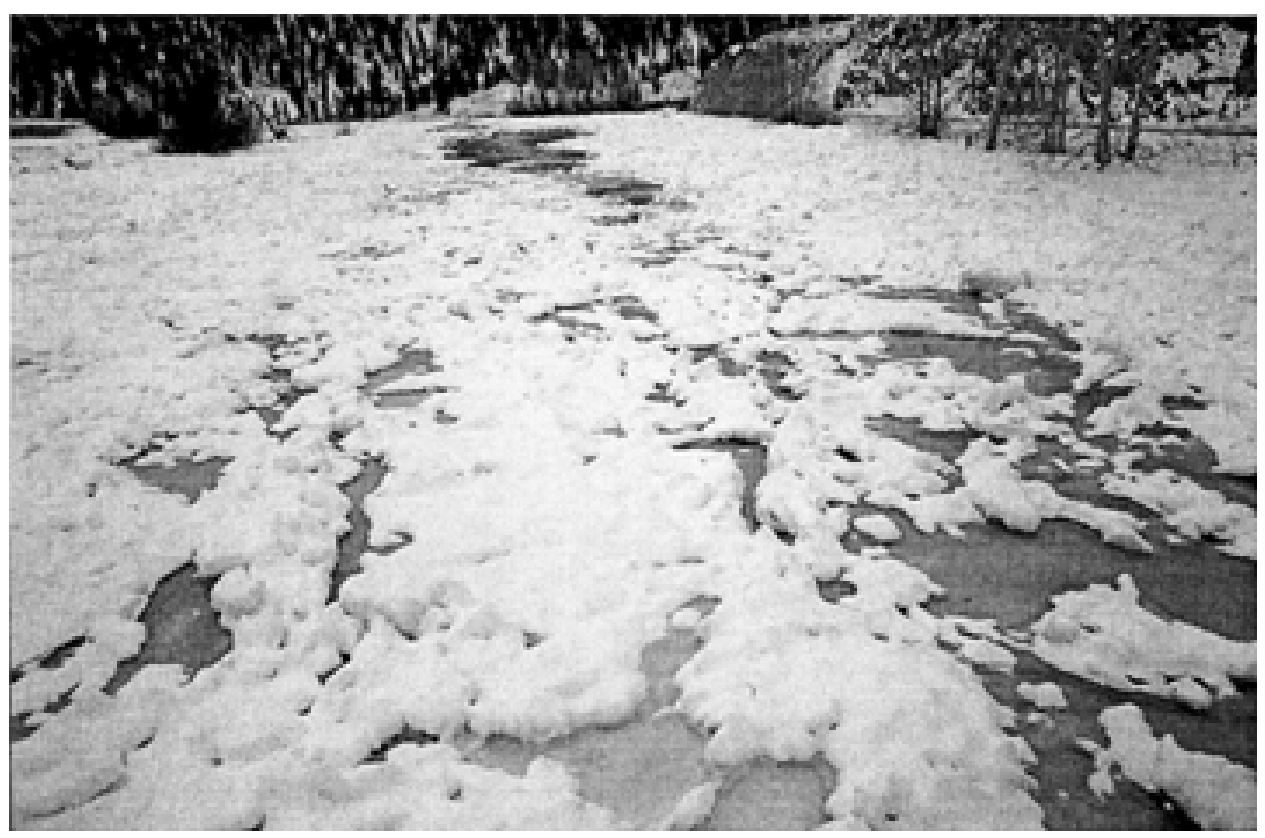

Figure 1. Ice jam at first signs of failure; view is looking downstream. 


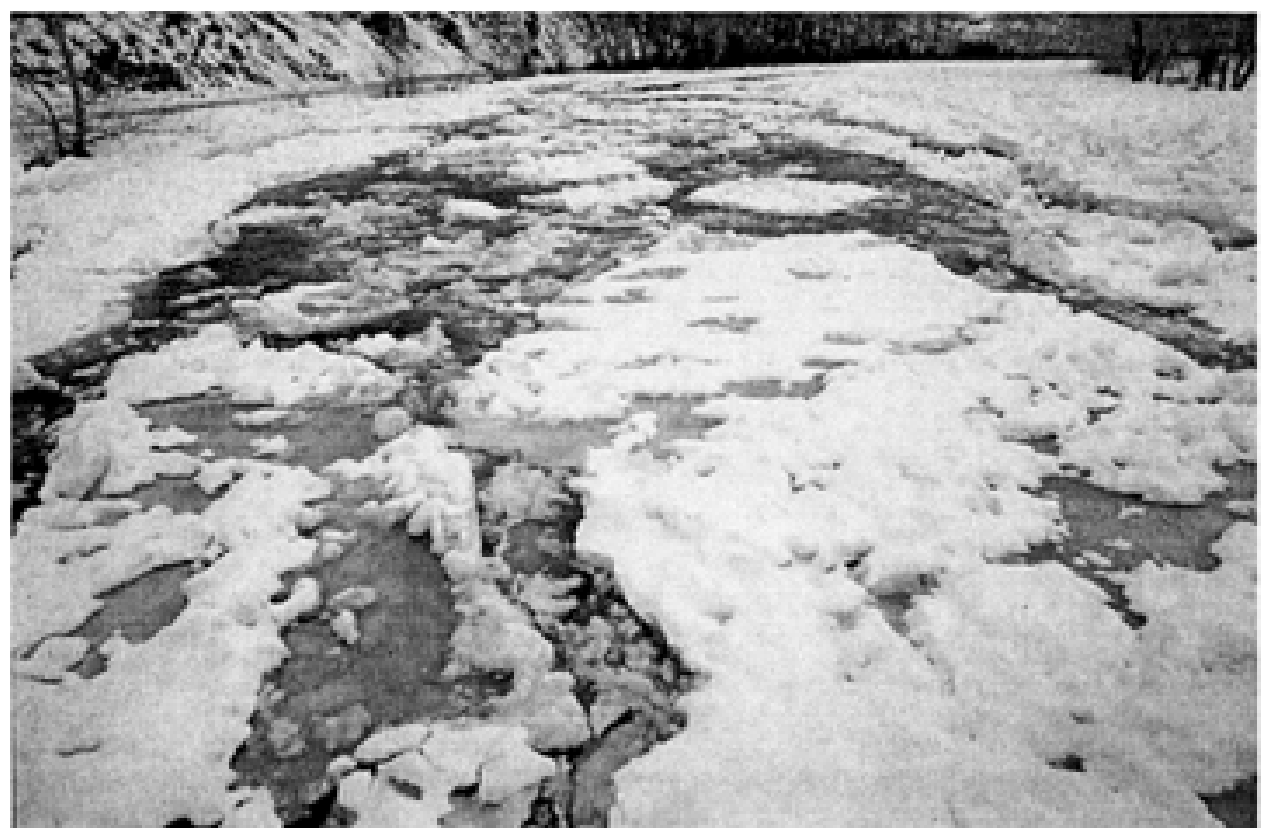

Figure 2. Ice jam failure at beginning of ice motion; view is looking upstream.

banks (Fig. 4). The shear walls were grounded on the bed. The unsteadiness observed is significant because the time lapsed since the water levels initially rose to the final passage of the ice from upstream was only about 15 minutes. Questions remain as to what happened to the ice as it traveled downstream, what caused the initial water level rise, and what combination of water and ice flow resulted in the initial accumulation.

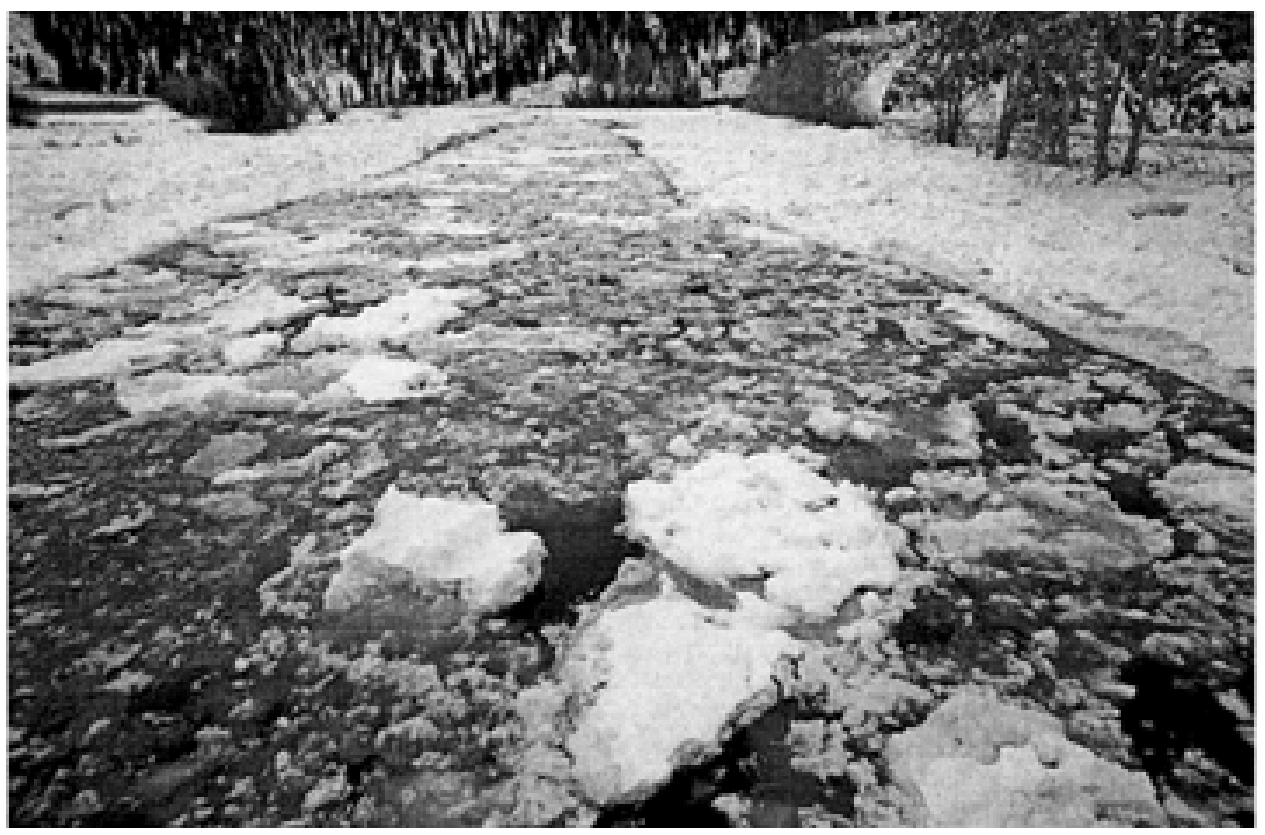

Figure 3. Ice jam failure with ice fully mobilized; view is looking downstream. 


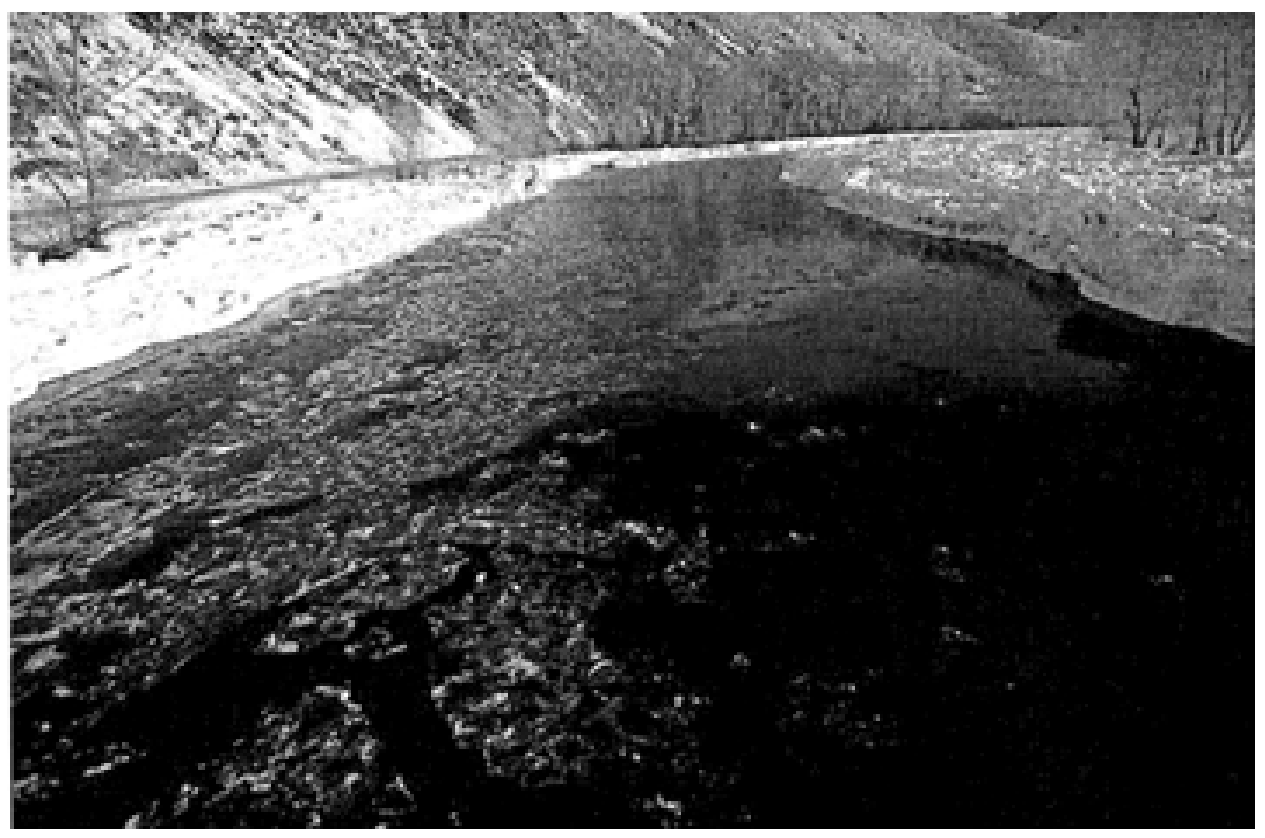

Figure 4. River channel following ice jam failure; view is looking upstream.

\section{Objective and approach}

The ultimate objective of this work is to address the three sets of questions presented in the Needs for Research section. To do so required carrying out the following tasks:

- Determine the temporal sequence of events associated with ice jam formation, failure, and reformation.

- Identify the important processes and parameters involved in shoving and thickening, and properly formulate the equations describing them.

- Develop a numerical model, correctly representing the shoving and thickening of ice, for use in examining the formation and evolution of jams, including freezeup and breakup jams. The model would be used further to investigate 1) the progression of freezeup jams formed at an ice boom; 2) the effects of a jam on hydraulics as the length of the jam increases and evolves by shoving and thickening; 3) the failure of an ice jam ascribable to increases in water discharge simulating the effects of hydropower releases or surges from the failure of upstream ice jams; and 4) the effects of ice momentum on the predicted thickness of jams.

- Identify a parameter that delineates when the effects of ice momentum become important for determining a jam thickness profile, and when a fully coupled, moving ice model should be used instead of a steady-state, stationary ice model.

\section{Discussion}

Although the published literature on jams contains descriptions of the general processes leading to shoving and thickening, there is no description of how jams in fact move and, in so doing, modify water flow. In very general terms, an ice jam forms when the downstream movement of ice is stopped. If the forces exerted on the jam continue to increase (owing to increased water discharge, increased cover 
length, or reduced jam strength), the jam eventually fails and moves downstream. If an area of channel downstream is encountered where the resisting forces on the moving ice are again great enough, the jam will reform. Existing models predict the equilibrium jam thickness, which is the constant thickness that would be expected in a uniform channel under conditions of steady flow when the resisting and downstream-acting forces are perfectly in balance. Those models assume that, when the net downstream-acting force reaches the level of passive pressure failure, the jam must thicken to withstand the forces. The unsteadiness of both the ice and water movement during a shoving and thickening event make the concept of equilibrium thickness questionable.

In contrast with jam formation, juxtaposition (or surface assembly) of ice floes is primarily a single-layer process that can be adequately described from hydraulics considerations, and from the size, shape, and distribution of ice pieces. Much work has addressed the problem of block underturning at the upstream edge of obstructions, and some work has addressed what happens to the blocks following underturning. Such cover-formation processes are easy to visualize in the laboratory and the river, as they generally occur at the water surface and entail the motion of single-layer ice floes coming into contact with a stationary obstruction. The juxtaposition of ice floes or the motion of an ice block at the upstream edge of an obstruction can be considered to be a fairly steady process because the effects of ice-piece movement on the hydraulics are minimal.

Shoving and thickening, however, are much more common in nature during the development of freezeup jams (made up of frazil slush or pans and small ice pieces), as well as during the formation and evolution of breakup jams. The manner whereby jams form and evolve is also important in determining how they fail. In comparison to the fairly steady water and ice motion during juxtaposition or underturning, the ice and water interaction during jam failure and thickening results in highly unsteady water and ice velocities, depths, and thicknesses.

\section{REVIEW OF ICE JAM MODELING}

It is convenient to review prior ice jam modeling in the context of the ways jams develop and are classified. This section presents an ice jam classification system, reviews past analyses of stationary jams, and briefly describes existing numerical models used for predicting jam thickness.

\section{Review of ice jam classification}

The International Association for Hydraulic Research (IAHR) Working Group on River Ice Hydraulics (IAHR 1986) published a state-of-the-art report classifying the different types of ice jams and reviewing techniques for their analysis. The report defines ice jams as stationary accumulations of fragmented or frazil ice that restrict flow. This broad classification could include any form of ice cover or accumulation, except for a thermally grown sheet ice cover. The classification system distinguishes ice jams by their season of formation, dominant formation process, spatial extent, and state of evolution. It is clear from the classification that jam formation, whichever type of jam forms, is intrinsically unsteady. Jams develop and adjust in thickness and extent in accordance with flow conditions, ice availability, and weather conditions. Also clear from the report, however, is that existing formulations of jams assume steady conditions, although unsteady water and ice move- 


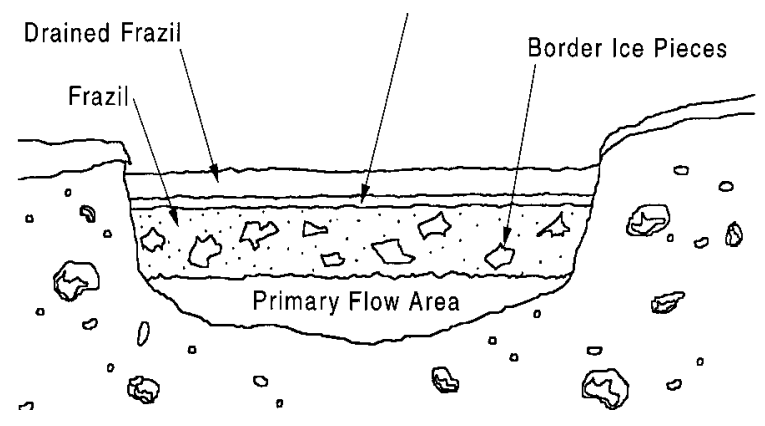

Figure 5. Cross section of a freezeup jam.

ment play important parts in the development of nearly all the jam types in the IAHR classification.

\section{Season of occurrence}

Ice jams are typically called freezeup or breakup jams, in accordance with the season in which they form. This classification suitably represents the hydrological and meteorological conditions prevailing during jam formation.

Freezeup jams form during periods of subfreezing air temperatures when frazil ice production is great. Their composition is mainly frazil and broken pieces of shore ice, as depicted in Figure 5. Frazil ice forms in areas of high water velocity and turbulence, where heat loss from the water surface is greatly increased. Some areas may remain open and produce frazil throughout the winter. Subfreezing air temperatures reduce basin runoff, resulting in fairly steady water discharge from base flow. The ice discharge varies as frazil production increases or freezeup jams form and cut off the supply of frazil to downstream reaches.

When frazil travels downstream, it agglomerates as slush, which rises to the water surface and forms ice pans. The pans may break upon passing through very

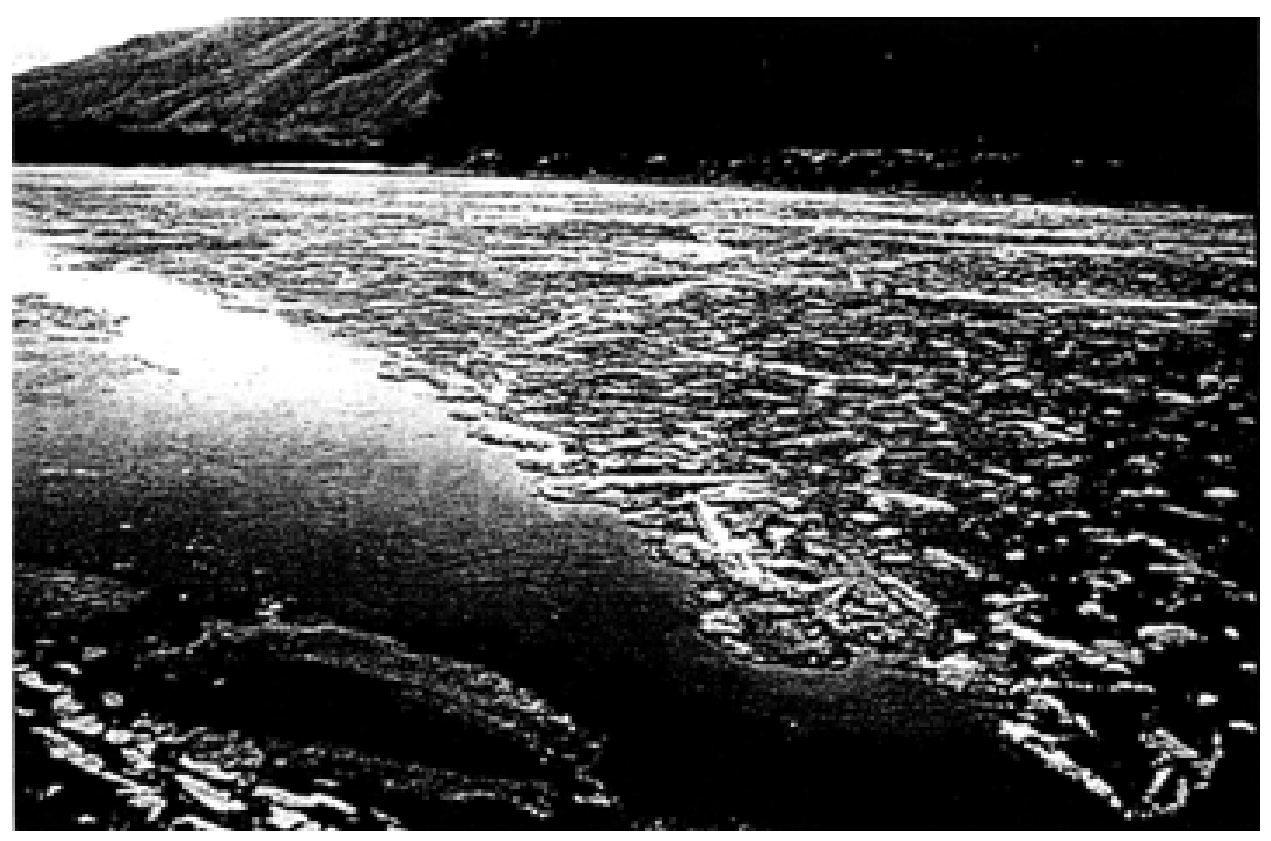

Figure 6. Surface jam resulting from juxtaposition of frazil pans. 


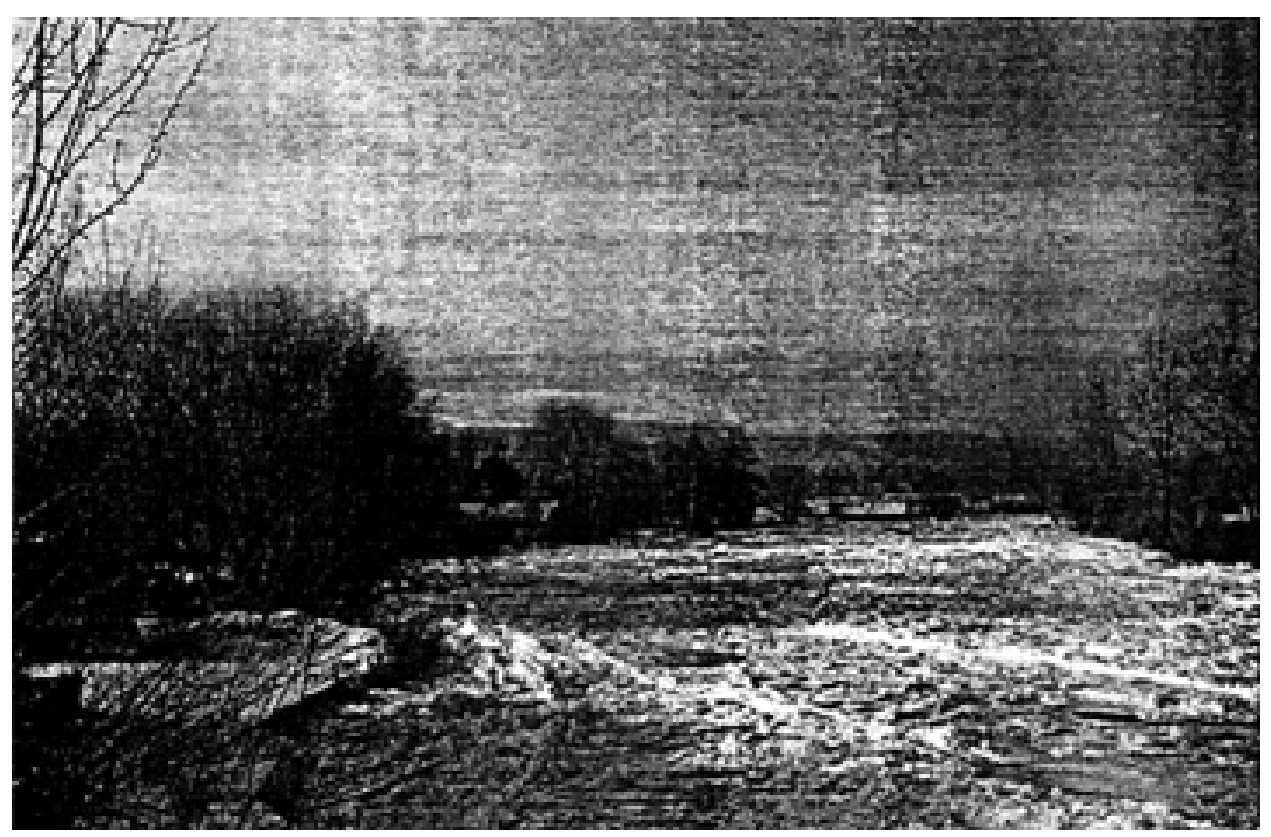

Figure 7. Freezeup jam following shoving and thickening.

turbulent areas of flow or strengthen by freeze-thickening. Pans may slow at channel constrictions or stop at downstream ice covers. Stopped and juxtaposed frazil pans form surface jams as shown in Figure 6. With continued transport of frazil into a reach, the length of a cover of juxtaposed ice pans may increase to the point where downstream forces exceed the cover's strength, causing shoving, collapse, and thickening of the cover. Figure 7 shows a freezeup jam formed by shoving and thickening of ice. Freezeup jams may strengthen by surface freezing, which usually causes them to be thinner than breakup jams formed at similar flow rates.

While water discharge may be steady during the formation and evolution of a freezeup jam, flow depth, ice velocity, and jam thickness are not. With continued frazil production, a freezeup jam may progress upstream with time, raising water levels as it progresses. Figure 8, for example, shows the temporal variation of the location of the upstream edge of a freezeup jam on the Salmon River in Idaho for the winter season 1990-91. The jam initiated on Julian Day 61 (30 November) and reached a maximum length of approximately $34 \mathrm{~km}$ (21 river miles). This freezeup jam is an annual occurrence and consistently initiates in a deep pool at river-mile 233. The upstream progression of the freezeup jam varies from year to year and depends on air temperature. Plotted in Figure 8 is the daily mean air temperature; frazil ice is generated when air temperature plunges below $0^{\circ} \mathrm{C}$. Water depths experience unsteady variations because of the shoving and thickening of the freezeup jam. Indeed, field measurements show that as the jam progresses through a reach, the water level increases by approximately $2 \mathrm{~m}$.

Breakup jams occur during periods of relatively warm weather and are typified by periods of increased runoff. The runoff results from snowmelt, rain, or groundwater release. For these jams, water discharge usually is highly unsteady with surges being possible owing to the failure and reformation of jams as breakup progresses downstream. Many anecdotal accounts of the highly unsteady nature of breakup jams have been presented (Moberley and Cameron 1929, p. 151). Moberley was the 
Figure 8. Temporal variation of air temperature and location of upstream edge of a freezeup jam on the Salmon River, Idaho, 1990-91.

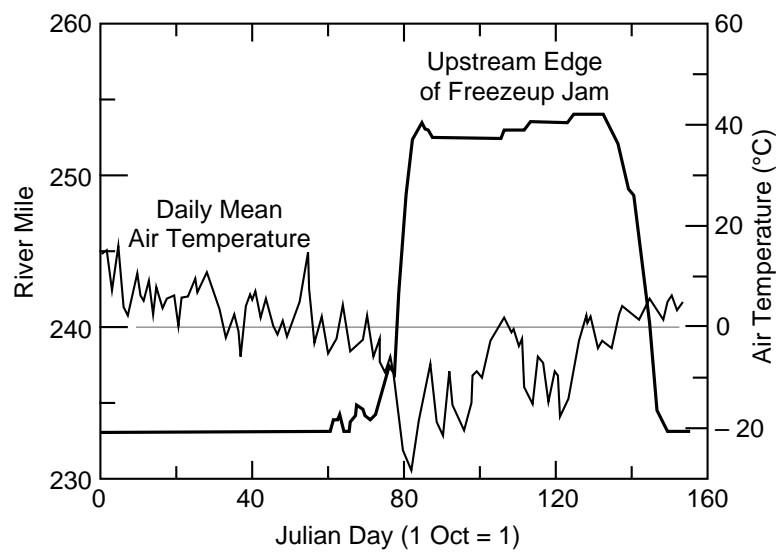

Factor of the Hudson's Bay Co. post at Fort McMurray in Alberta and described the following events on the morning of 20 April 1875:

...The winter of 1874-75 was a bitter one, with deep snow and never a thaw until April. On the 2nd or 3rd of that month, however, a further heavy fall of snow was followed by a sudden rise in temperature. The change of weather and the weight of melting snow caused the ice for the 85 mile stretch of rapids above the fort [Fort McMurray] to breakup, and it came down the Athabasca with terrific force. On striking the turn of the stream at the post it blocked the river and drove the ice 2 miles up the Clearwater [a major tributary] in piles 40 to 50 feet high. In less than an hour the water rose 57 feet, flooding the whole flat and mowing down trees, some $3 \mathrm{ft}$. in diameter, like grass....

As its name implies, a breakup jam consists of pieces of broken sheet ice, refrozen frazil ice, and brash or slush ice. Figure 9 depicts a fairly typical breakup jam. Freeze-bonding of ice pieces is usually negligible for a breakup jam, because the above-freezing air in which they form inhibits it. Breakup jams typically form at reaches where the downstream progression of a run of moving ice (or breaking front) slows because of reductions in channel slope, or width, or where it encounters resistant portions of ice cover, such as locations of freezeup accumulations. The severity of flooding during breakup jams depends on many factors, such as initial ice cover thickness and strength, characteristics of the runoff hydrograph, and, relatedly, weather. Gradually warming weather with no rain, for instance,

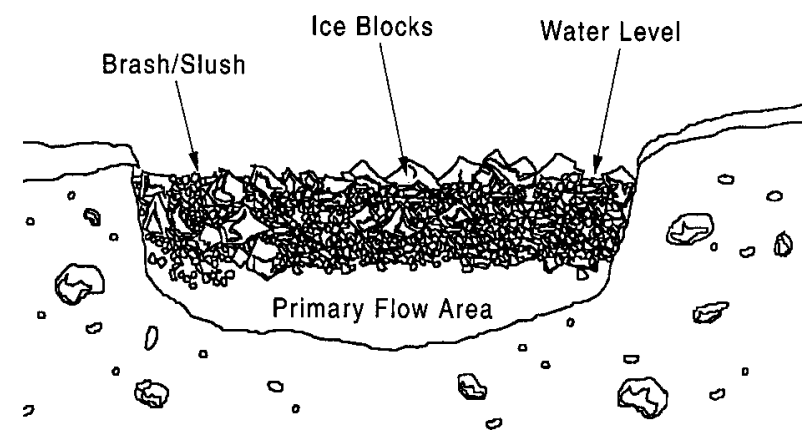

Figure 9. Cross section of a breakup jam. 


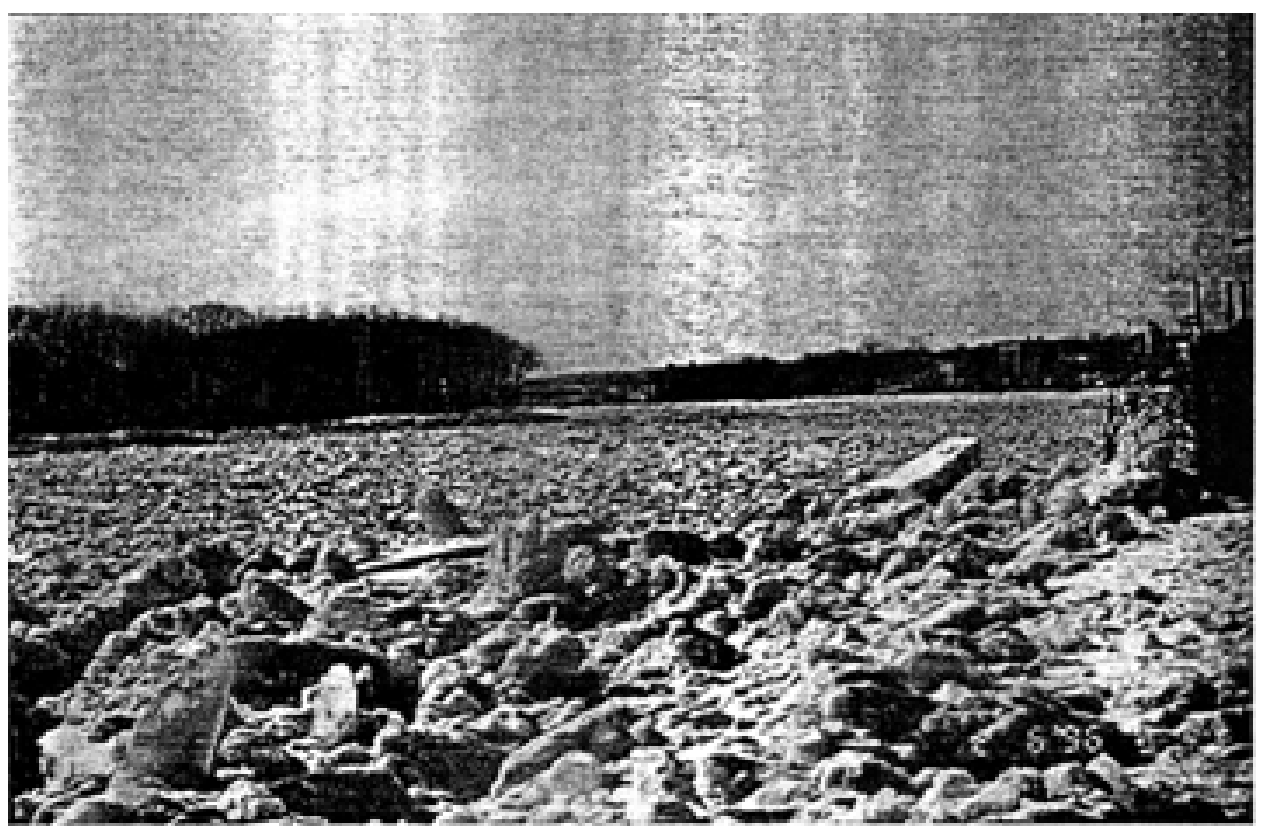

Figure 10. Mid-winter breakup jam on the Kennebec River in Maine.

mildly increases runoff discharge and decreases ice strength, often resulting in less severe jamming and flooding.

Conversely, mid-winter jams happen with the onset of mid-winter thaws (usually in January for the northern U.S., but also in early February, and late December). Rain and snowmelt runoff on impermeable frozen ground can result in very steep increases in river discharge that break up relatively strong ice covers. While ice covers in early to mid-winter are typically not as thick as they might be in late winter or early spring, mid-winter jams can produce severe flooding. Also, since the weather systems bringing mild mid-winter weather are usually short-lived, and are soon followed by frigid weather, these jams may remain in place, consolidating as virtually a monolithic mass of ice. This sets the stage for additional problems later during the normal breakup period. Figure 10 shows a mid-winter breakup jam on the Kennebec River in Maine. It formed during January 1996 at a peak discharge of approximately $2000 \mathrm{~m}^{3} / \mathrm{s}$. Once the flow receded to the river's normal winter levels of 200 to $300 \mathrm{~m}^{3} / \mathrm{s}$, the jam grounded in many locations and continued to cause increased water levels upstream.

As a further example of the highly unsteady nature of breakup jams, Figure 11 shows the stage hydrograph for a gauging station on the St. John River in northern Maine, where breakup jams are an annual occurrence. Superimposed on the general rise in river stage are several short-duration peaks attributable to ice jam formation and failure. Additional instrumentation installed at the gauge site identified the initial time of failure of the sheet ice cover as 0610 on 22 April 1994, which corresponds to the stage drop following the first large peak. Subsequent peaks are most likely ascribable to reformation and failure of additional jams downstream from the gauge or from surges due to failure of jams upstream from the gauge. Field observations have shown that jams do form at locations approximately $1,4,8$, and $15 \mathrm{~km}$ downstream from the gauge. 
Figure 11. Stage record for USGS station no. 0101000 on the St. John River in Maine during 1994 breakup.

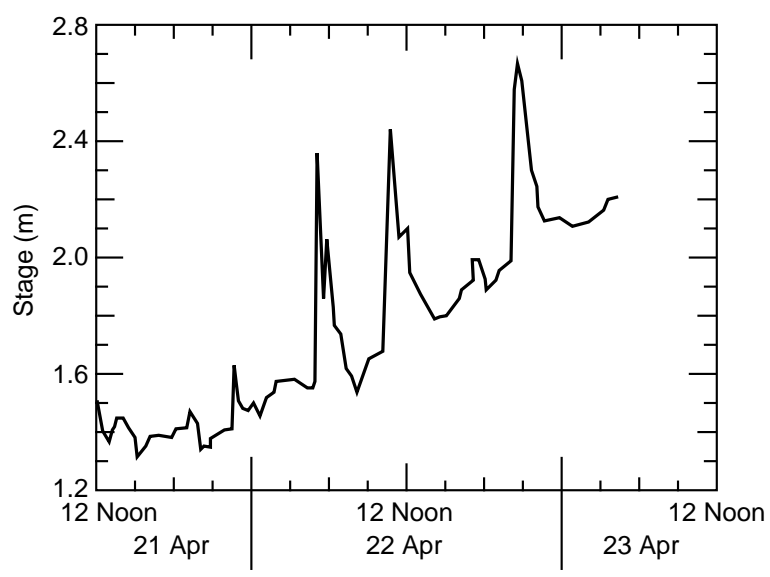

\section{Dominant formation process}

Ice jams are also classified in terms of the processes dominating their formation. The important processes are transport and deposition of ice, congestion of ice, and shoving and thickening of ice.

Jams known as "hanging dams" are local, thick accumulations of frazil ice. Frazil ice arriving at the upstream edge of an existing ice cover may submerge and travel beneath the cover until reaching an area of lower water velocity, where it deposits on the underside of the cover. These formations, or hanging dams, may continue to develop during the entire winter season, growing up to thicknesses of $20 \mathrm{~m}$. Large hanging dams may constrict flow and block the downstream passage of ice, occasionally initiating more severe breakup jams.

A congestion or surface jam forms when ice transport along the water surface is reduced by shore ice growth, transverse floating objects, or channel constrictions, such as bridges. This initiation method is typical of both freezeup and breakup jams. Water flow is fairly steady for congestion jams, which, initially at least, are relatively thin (i.e., single floe thickness).

A submergence or frontal progression type of jam typically forms at some transverse floating barrier, such as an intact ice cover. Arriving ice floes tip and submerge, but come to rest almost immediately at the upstream edge of the obstruction, causing the jam to progress upstream. This type of jam, often called a narrow channel jam, primarily forms during the freezeup of contacting frazil pans.

Given a steady ice supply from upstream, both the congestion and frontal progression type of jams may grow to a point beyond which they are not able to withstand the increased streamwise load exerted by additional ice conveyed to the jam or by increased discharge. Then, shoving and thickening occur. A jam formed by shoving and thickening is sometimes termed a wide channel jam. It will remain in place as long as the downstream acting forces of water shear and gravity (and possibly wind shear) can be resisted by the jam's strength and resistive shear stresses acting between the ice and the banks. If the load (water drag and ice weight) becomes too great, the jam must thicken to increase its resistance to downstream movement. Thickening increases both the jam's strength and the shear stress between the jam and the bank.

Once a jam collapses, thickens, and possibly moves downstream, jam reformation becomes highly unsteady. The shear stress exerted on the jam underside reduces as it begins moving, which in turn increases water velocity and reduces 
depth. Ice acceleration produces an ice momentum that must be overcome to arrest ice motion at a location downstream where thickening takes place. During shoving and thickening of accumulating ice, water depth and velocity, ice thickness, and ice velocity are all interrelated and vary with distance and time. It is this unsteady nature of shoving and thickening that this study addresses.

Spatial extent

The horizontal and vertical extent of a jam are also used to classify jam type. In plan, jams are either partial or complete, according to their extent across a river. A partial jam means that a portion of the river width remains as an intact ice sheet, or one channel around an island jams while the other remains clear. In vertical section, jams are classified as floating or grounded. Grounding, when ice extends to the channel bed, takes place quite often near the riverbanks, at shallow areas such as bars or crossings, and near the toe region of a thick jam. Grounded jams usually result from very unsteady water and ice flows. They severely limit water flow, greatly increasing water levels. Water may flow as seepage through grounded accumulations or even over the top of a jam. Little is known about the mechanism of grounding or the permeability of grounded jams. Floating jams are more common and easier to analyze, though they may become partially grounded when river flow recedes. Most analyses to date assume floating jams, whose flotation follows hydrostatic pressure law.

State of evolution

The final classification category is that describing the state of jam evolution: steady-state, evolving in time, or evolving up-channel. An evolving jam continues to be subject to unsteady flow rates, ice discharges, or changes in other ice variables (such as strength). A breakup jam, already formed and undergoing shoving and thickening, will continue evolving with nonuniform thickness, depth, and water velocities. A freezeup jam may experience fairly steady flow rates, but frazil ice production and transport may cause it to shove and thicken with time. Figure 12 shows ice jam evolution with time. As the jam thickens and progresses upstream, water levels rise and velocities decrease. Whatever the final water surface level and jam thickness profile might look like, the ice thickness and velocity are not steady as the jam develops.

If conditions do become steady, uniform, and stable, a jam may have an equilibrium section. Strictly speaking, an equilibrium section is uniform only in a reachaveraged sense. Figure 12d shows that the thickness and depth are nearly constant in the longitudinal direction. Moreover, the bed slope, water-surface slope, and energy slope are equivalent in the equilibrium section. These conditions of uniformity were assumed by ice researchers when formulating the first analyses of forces exerted on a stationary jam (e.g., Pariset and Hauser 1961, Uzuner and Kennedy 1976).

\section{Analysis of stationary jams}

A major advance in addressing the effects of ice jams on water levels, and in estimating jam thicknesses, was the realization that a floating jam could be likened to a granular material contained between two parallel walls. The behavior of a granular material is influenced by the forces exerted upon it and its material properties. As the length of a jam increases, these forces increase, as do stresses within the jam. 

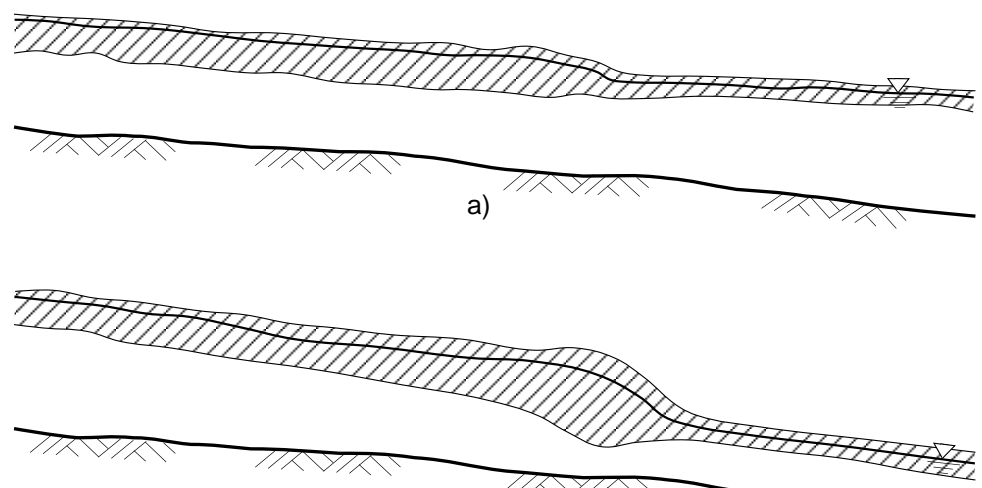

b)

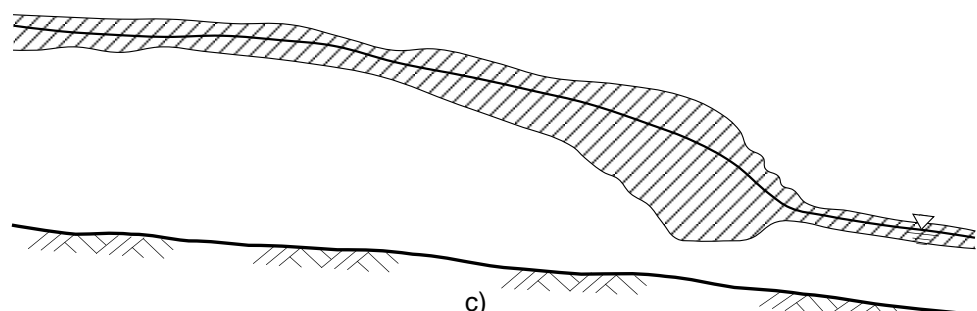

c)

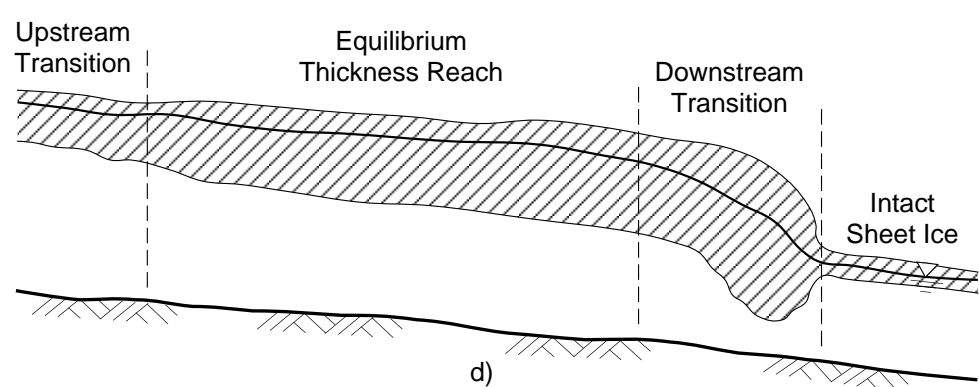

Figure 12. Idealization of ice jam evolution with time.

The first analysis was done by the Canadian R.J. Kennedy (1958), who was interested in the forces exerted on a boom by a pulpwood jam. To determine those forces, he developed the following relation

$$
B \frac{d F}{d x}+\tau_{\mathrm{i}} B-2 k_{0} \lambda F=0
$$

where

$B=$ width of the holding area

$\tau_{\mathrm{i}}=$ shear stress on the underside due to water flow

$k_{0}=$ coefficient of lateral thrust

$\lambda=$ coefficient of friction of the pulpwood against the shore boundary

$F=$ force per unit width acting in the downstream direction ( $x$-direction).

If there exists a force $f_{1}$ exerted by the water impinging on the upstream end of the accumulation, then eq 1 can be integrated to obtain the force at any location $x$ (measured from the upstream end of the accumulation), i.e.

$$
F=\frac{B \tau_{\mathrm{i}}}{2 \lambda k_{0}}+\left(f_{1}-\frac{B \tau_{\mathrm{i}}}{2 \lambda k_{0}}\right) \exp \left[\frac{-2 \lambda k_{0} x}{B}\right] .
$$


Equation 2 shows that, as the length of the accumulation increases, the force level reaches an asymptotic value that is related only to the water drag and cover width (friction and lateral stress coefficients being constant). Kennedy's formulation, however, neglected the streamwise component of the weight of the pulpwood and assumed that the depth (and therefore water shear) was constant. Consequently, the force through the jam would be independent of the thickness and bulk density of the pulpwood accumulation.

Berdennikov (1964) investigated the forces exerted on an ice boom retaining an ice accumulation. While he initially identified the weight component of the ice mass parallel to the water surface as one of the forces to be considered, he dismissed this force and the hydrodynamic pressure against the leading edge of the cover $f_{1}$ as being so small as to be negligible. His expression for the normal stress in the ice field $\sigma_{\mathrm{x}}$, assuming that $\sigma_{\mathrm{x}}=0$ at $x=0$, is

$$
\sigma_{\mathrm{x}}=\frac{B \tau_{\mathrm{i}}}{2 \lambda k_{0} \eta}\left(1-\exp \left[\frac{-2 \lambda k_{0} x}{B}\right]\right) \text {. }
$$

Pariset and Hausser (1961), then Pariset et al. (1966), advanced Kennedy's formulation by including the streamwise components of the weight of the cover and an assumed "cohesive" stress acting between the ice and the banks. Summation of the forces acting on the ice cover (Fig. 13) gives

$$
d F B+2\left(\tau_{\mathrm{c}} \eta+\lambda k_{0} F\right) d x=\left(\tau_{\mathrm{i}}+f_{3}\right) B d x
$$

where

$$
\begin{aligned}
F= & \text { force per unit width acting in the downstream direction } \\
\tau_{\mathrm{c}}= & \text { cohesive stress per unit area at the banks } \\
\eta= & \text { cover thickness } \\
f_{3}= & \text { downstream component of the weight of the cover per unit area and } B, k_{0}, \\
& \lambda \text {, and } \tau_{\mathrm{i}} \text { are defined as above, so that } \\
f_{3}= & s_{\mathrm{i}} \rho g \eta S
\end{aligned}
$$

where

$$
\begin{aligned}
& s_{\mathrm{i}}=\text { specific gravity of ice } \\
& \rho=\text { density of water } \\
& g=\text { acceleration due to gravity } \\
& S=\text { slope }
\end{aligned}
$$

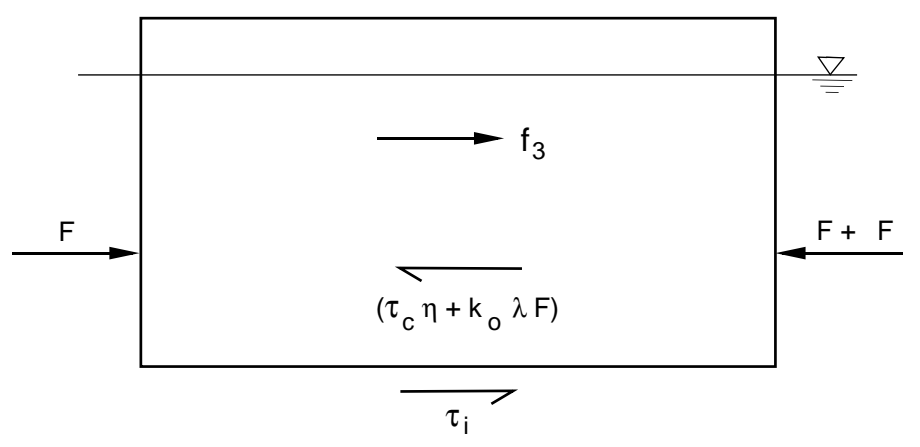

Figure 13. Forces acting on an ice cover. (After Pariset et al. 1966.) 
Because their formulation is for a uniform channel, bed slope, water surface slope, and energy slope are taken as equal. Integration of eq 4 results in an expression for the longitudinal force per unit width as a function of the distance from the upstream edge of the cover, similar to eq 2, i.e.

$$
F=\frac{B}{2 k_{0} \lambda}\left(\tau_{\mathrm{i}}+f_{3}\right)-\frac{\tau_{\mathrm{c}} \eta}{k_{0} \lambda}-\left\{\frac{B}{2 k_{0} \lambda}\left(\tau_{\mathrm{i}}+f_{3}\right)-\frac{\tau_{\mathrm{c}} \eta}{k_{0} \lambda}-f_{1}\right\} \exp \left[\frac{-2 k_{0} \lambda x}{B}\right]
$$

where $f_{1}$ is as defined above. Equation 6 is based on an assumed "equilibrium thickness" of an ice jam over steady, uniform flow. Pariset et al. also suggest definitions for narrow and wide jams. When the term within the large brackets of eq 6 (the multiplier of the exponential term) is negative, the longitudinal force $F$ is a maximum at the upstream edge of the cover $(x=0)$. This is the case for so-called narrow jams. As the cover progresses upstream, the downstream thrust is resisted by shear stress at the banks, which grows faster than the additional hydrodynamic forces exerted on the jam. Conversely, when the term within the brackets is positive, the longitudinal force $F$ grows with distance downstream from the upstream edge of the jam, asymptotically approaching a maximum as the distance grows very large. This maximum longitudinal force acting through a wide jam is

$$
F_{\max }=\frac{B}{2 k_{0} \lambda}\left(\tau_{i}+f_{3}\right)-\frac{\tau_{c} \eta}{k_{0} \lambda} .
$$

Pariset et al. recognized that this maximum force (or sum of external forces) is resisted by the strength of the accumulated ice, which is assumed to behave as a granular material. If $F_{\max }$ exceeds jam strength, the jam fails and must thicken until there is a balance between the external forces and jam strength. They likened the maximum strength of the ice jam to that of a granular material under complete mobilization of the passive pressure resistance, i.e.

$$
K_{\mathrm{p}} s_{\mathrm{i}} \rho g\left(1-s_{\mathrm{i}}\right) \frac{\eta^{2}}{2}=\tan ^{2}\left(\frac{\pi}{4}+\frac{\phi}{2}\right) s_{\mathrm{i}} \rho g\left(1-s_{\mathrm{i}}\right) \frac{\eta^{2}}{2}
$$

where $K_{\mathrm{p}}$ is a passive pressure coefficient and $\phi$ is the angle of internal resistance of the accumulated broken ice, and is commonly taken as the angle of repose for granular materials. Pariset et al. then equated jam internal stress to the sum of the stresses exerted by the external forces. In doing so, they introduced the coefficient $\mu$, where

$$
\mu=k_{0} K_{\mathrm{p}} \lambda
$$

which combines the ice properties into one coefficient. The stress balance results in an equation relating jam thickness and stresses exerted against the jam:

$$
s_{\mathrm{i}} \rho g\left(1-s_{\mathrm{i}}\right) \frac{\eta^{2}}{2}=\frac{B}{2 \mu}\left(\tau_{\mathrm{i}}+f_{3}\right)-\frac{\tau_{\mathrm{c}} \eta}{\mu} .
$$

The shear stress $\tau_{\mathrm{i}}$ in eq 10 is expressible as

$$
\tau_{\mathrm{i}}=\rho g \frac{u^{2}}{C^{2}}
$$

where $u$ is water velocity beneath the cover and $C$ is the Chezy coefficient. Pariset et al. assumed that values of $C$ are essentially equivalent for the ice surface and the bed. 
The weight of the cover in the downstream direction $f_{3}$ can be defined as

$$
f_{3}=s_{\mathrm{i}} \rho g \eta \frac{u^{2}}{C^{2} R_{\mathrm{H}}}
$$

where $R_{\mathrm{H}}$ is the total hydraulic radius beneath the ice. Substituting eq 11 and 12 into 10 , and dividing by $H^{2}$ ( $H=$ the open water depth just upstream of the ice cover), renders eq 10 dimensionless, i.e.

$$
\frac{B u^{2}}{\mu C^{2} H^{2}}\left[1+s_{\mathrm{i}} \frac{\eta}{R_{\mathrm{H}}}\right]=\frac{2 \tau_{\mathrm{c}} \eta}{\rho g \mu H^{2}}+s_{\mathrm{i}}\left(1-s_{\mathrm{i}}\right) \frac{\eta^{2}}{H^{2}} .
$$

Equation 13 can be used to predict ice thickness for the wide-jam case. Pariset et al. recognized that freeze-bond forces are of lesser importance for thicker jams, and are not important during breakup conditions, because jam resistance is dominated by gravitational effects, i.e., ice weight. They developed a dimensionless stability parameter $X$ to relate jam thickness to upstream open-water conditions:

$$
X=\frac{Q^{2}}{B C^{2} H^{4}}=\frac{B u_{\mathrm{o}}{ }^{2}}{C^{2} H^{2}}=\frac{\mu s_{\mathrm{i}}\left(1-s_{\mathrm{i}}\right)\left(\frac{\eta}{H}\right)^{2}\left(1-s_{\mathrm{i}} \frac{\eta}{H}\right)^{3}}{\left(1+s_{\mathrm{i}} \frac{\eta}{H}\right)}
$$

where $Q$ is water discharge and $u_{\mathrm{o}}$ is bulk open water velocity for uniform flow far upstream of the jam. Values of $X$ are plotted in Figure 14 for $s_{i}=0.92$ and $\mu=1.28$, which are values adopted by Pariset et al. for jams in the St. Lawrence River. The figure indicates jams as being stable (inside the bell curve) or unstable (outside the curve). The curve is useful, but it does not enable direct calculation of ice thickness. Moreover, it assumes equilibrium conditions, i.e., steady, uniform flow of water and uniform ice thickness.

Uzuner and Kennedy (1976) presented a detailed formulation of the timedependent differential equations describing the force equilibrium in a static, floating ice jam. Their formulation is

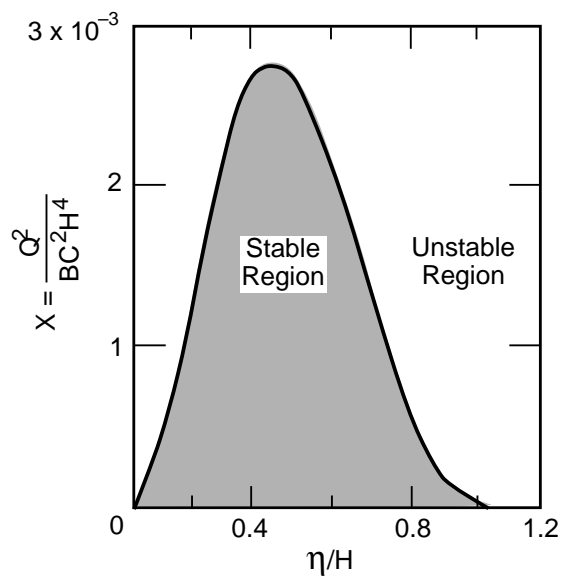

Figure 14. Dimensionless stability parameter. (After Pariset et al. 1966.) 


$$
\frac{\partial}{\partial x}\left(\eta \sigma_{x}\right)-\frac{\partial}{\partial y}\left(\eta \tau_{x y}\right)-\tau_{i} \cos \left(s_{i} \frac{\partial \eta}{\partial x}\right)-s_{i} \rho g \eta \sin (\theta+\alpha)=0
$$

where

$\sigma_{\mathrm{x}}=$ normal stress in the streamwise direction

$\tau_{\mathrm{xy}}=$ shear stress at the banks

$\tau_{\mathrm{i}}=$ shear on the underside of the cover

$\theta=$ slope of the bed

$(\theta+\alpha)=$ slope of the water surface.

Uzuner and Kennedy expressed $\sigma_{\mathrm{x}}$ and $\tau_{\mathrm{xy}}$ as functions of the average vertical stress $\overline{\sigma_{z}}$ within the cover

$$
\overline{\sigma_{\mathrm{z}}}=\frac{\eta}{2} s_{\mathrm{i}} \rho g\left(1-s_{\mathrm{i}}\right)(1-p) \cos (\theta+\alpha)=\gamma_{\mathrm{e}} \eta
$$

where $p$ is jam porosity and $\gamma_{\mathrm{e}}$ is the equivalent unit weight of the jam. The Rankine and Mohr-Coulomb stress theories for granular materials give

$$
\sigma_{\mathrm{x}}=K_{\mathrm{p}} \overline{\sigma_{\mathrm{z}}}
$$

and

$$
\tau_{\mathrm{xy}}=C_{\mathrm{o}} \overline{\sigma_{\mathrm{z}}}+C_{\mathrm{i}}
$$

where

$$
\begin{aligned}
& K_{\mathrm{p}}=\text { passive pressure coefficient } \\
& C_{\mathrm{o}}=\text { shear stress coefficient } \\
& C_{\mathrm{i}}=\text { assumed cohesive intercept. }
\end{aligned}
$$

Substitution of eq 16 through 18 into 15, integration of the modified equation, eq 15 , then normalization using $x_{\mathrm{o}}=x / h_{\mathrm{n}}$ and $\eta_{\mathrm{o}}=\eta / h_{\mathrm{n}}$, yields

$$
\eta_{\mathrm{o}} \frac{\partial \eta_{\mathrm{o}}}{\partial x_{\mathrm{o}}}=k_{1}+k_{2} \eta_{\mathrm{o}}+k_{3} \eta_{\mathrm{o}}^{2}
$$

where

$$
\begin{aligned}
& k_{1}=\frac{\tau_{\mathrm{i}}}{2 K_{\mathrm{p}} \gamma_{\mathrm{e}} h_{\mathrm{n}}} \\
& k_{2}=\frac{s_{\mathrm{i}} \rho g S_{\mathrm{o}}-2 \frac{C_{\mathrm{i}}}{B}}{2 K_{\mathrm{p}} \gamma_{\mathrm{e}}} \\
& k_{3}=-\frac{C_{\mathrm{o}} h_{\mathrm{n}}}{K_{\mathrm{p}} B} \\
& h_{\mathrm{n}}=\left(\frac{f_{\mathrm{b}} q_{\mathrm{n}}^{2}}{8 g S_{\mathrm{o}}}\right)^{\frac{1}{3}} .
\end{aligned}
$$

Also

$S_{\mathrm{o}}=$ bed slope $(\sin \theta)$

$f_{\mathrm{b}}=$ Darcy-Weisbach resistance factor of the bed

$q_{\mathrm{n}}=$ unit discharge at a location upstream where ice does not affect the flow. 
The Uzuner and Kennedy formulation of the force balance associated with gradually varied, unsteady water flow was too complex for a general solution. They did show that, for a condition of quasi-steady jam formation in which the jam progresses upstream at a constant rate, the unsteady water flow equations are constant with time. For the section of the jam considered to be in equilibrium, eq 19 can easily be solved for $\eta_{\mathrm{o}}$ using the quadratic formula by setting the term on the left-hand side to zero.

The formulations proposed by Pariset et al. and Uzuner and Kennedy compose the basis for most subsequent analyses of static ice jams. Beltaos (1983), most notably, adapted the formulations for wide-river jams and expressed flow depth beneath a jam $h$ as

$$
h=q^{\frac{2}{3}}\left(\frac{4 g S_{\mathrm{o}}}{f_{\mathrm{O}}}\right)^{-\frac{1}{3}}
$$

where $f_{\mathrm{o}}$ is a composite value of the Darcy-Weisbach resistance coefficients for the bed and the jam underside. Solution of eq 10 for jam thickness, assuming that cohesion is negligible, and that $f_{3}$ is given by eq 5 , yields

$$
\eta=\frac{B S_{\mathrm{o}}}{2 \mu\left(1-s_{\mathrm{i}}\right)}\left\{1+\left[1+\frac{\left(2 f_{\mathrm{o}}\right)^{\frac{1}{3}} \mu\left(1-s_{\mathrm{i}}\right)}{s_{\mathrm{i}}}\left(\frac{f_{\mathrm{i}}}{f_{\mathrm{o}}}\right)\left(\frac{{\frac{q^{2}}{g S_{\mathrm{o}}}}^{\frac{1}{3}}}{B S_{\mathrm{o}}}\right]^{\frac{1}{2}}\right\}\right.
$$

where $f_{\mathrm{i}}$ is a Darcy-Weisbach resistance factor for flow along the jam underside. Beltaos also presented field data, consisting of thickness measurements for several ice jams that had refrozen in place. Using eq 25, he back-calculated values of $\mu$ and found them to range from 0.6 to 3.5 , with these upper and lower limits obtained for conditions of considerable uncertainty. If the two extreme values are excluded, his data show consistently that $\mu=0.8$ to 1.3 . Beltaos found, on average, that $\mu \cong 1.2$, which is in good agreement with the value of 1.28 suggested by Pariset et al.

\section{Numerical modeling}

Several numerical models of jams have been developed. They assume that a balance exists between forces acting on the jam, predict equilibrium jam thickness, and estimate jam effects on water levels. Existing open-water models for steady and unsteady flow simulations have been adapted by the use of equations similar to eq 25 to provide estimates of ice jam conditions. Other models have been developed using steady or unsteady water flow and equilibrium (uniform) or nonequilibrium thickness.

HEC-2, the step-backwater program developed by the U.S. Army Corps of Engineers, was modified to include an ice cover (HEC 1979). In its initial version, the cover, or jam, was treated simply as a boundary, floating at hydrostatic pressure, that provides an additional resistance to flow at the water surface. The cover is taken as being static, with the user of the program inputting values of cover thickness, roughness, location, specific gravity, and a value of $\mu$. The program calculates 
the ice-affected water levels with the input configuration of the cover and also determines if the cover is stable according to the stability parameter proposed by Pariset et al. To generate an equilibrium thickness profile, many iterations are necessary with modifications made to the ice thickness and roughness values.

DWOPER, an unsteady flow forecasting model developed by the U.S. National Weather Service, was also adapted to investigate the effects of ice covers on water levels. Daly and Ashton (1983) modified the St. Venant equations describing the unsteady water flow to include the frictional resistance of the ice to the water flow. They concentrated on running steady water discharges with a stationary ice cover and then instantaneously removing the cover, simulating a complete cover failure and passage downstream. The ensuing transients increased with increasing bed slopes as would be expected. Their work did not include nonuniform covers or jams and they pointed out the necessity of including ice motion in developing a truly unsteady ice jam model.

Flato and Gerard (1986) and Flato (1987) applied their model, ICEJAM, to produce ice jam profiles for a range of steady-flow discharges. As it uses a form of the differential equation describing the balance of forces on the ice cover, similar to that of eq 19, it can be used to describe the complete thickness profile even if there is not an equilibrium section. The input data necessary to run ICEJAM include water discharge, ice jam characteristics (bulk specific gravity, angle of internal resistance, and porosity), channel data, roughness of the bed and ice, and initial estimates of water depth and jam thickness. The model first calculates the normal depth (under ice) profile based on the initial estimates of ice thickness. It then solves the ice force balance equation in a forward-difference mode, stepping downstream from the upstream end of the jam. The hydraulic conditions are then modified for these new ice thicknesses by means of the standard step-backwater calculation technique moving in an upstream direction. Iterations of the ice and water calculations continue until an acceptable tolerance is met. Adjustments are made in the ice thickness at the toe of the jam in relation to a prescribed ice erosion velocity. The model produced reasonable and stable results when a damping factor of $1 / 3$ was applied to the calculated corrections for ice thickness.

RIVJAM is a model developed by Beltaos (1993). It is based on a similar model proposed by Beltaos and Wong (1986). Both models use a steady water discharge and include the seepage flow through the jam in an attempt to better define ice thickness near the toe of the jam, which may be grounded. RIVJAM solves firstorder differential equations for the water depth beneath the cover and the ice thickness. It does so by means of a predictor-corrector scheme, and the solution procedure may progress in an upstream or downstream direction. Beltaos (1993) showed that RIVJAM was able to reproduce ice thickness profiles for a variety of nonequilibrium and potentially grounded jams quite well, with appropriate choices of several model parameters. The most tenuous of these appears to be the seepage coefficient, which is similar in concept to hydraulic conductivity (with units of length/time) for high Reynold's number flows. The model, however, does not include the unsteady movement of the ice cover and thus cannot include the effects of ice momentum, which would be important in cases of grounded jams.

A utility program was developed by Wuebben et al. (1995) for use with HEC-2 to simplify calculation of ice-affected water levels. The program, dubbed ICETHK, uses standard output variables from a HEC-2 simulation and calculates ice thickness based on an equation similar to eq 25. There are several calculation options, such as width smoothing, ice thickness smoothing, and overbank ice and rough- 
ness coefficient assignment as a function of thickness based on the data of Nezhihovskiy (1964). The program then automatically updates the HEC-2 input file to reflect these new values of ice thickness and roughness. Iterations continue until a specified tolerance is met. Considerable judgment is necessary in the jamtoe area, where ice thickness conditions cannot be expressed adequately by the equilibrium thickness as provided by eq 25 .

Lal and Shen (1991) developed the jam model RICE, which is intended to simulate unsteady conditions of water flow, water temperature, ice concentration, and thermal growth and decay of ice. In their model, ice travels downstream at the water velocity until it reaches some location where a jam forms, by either ice-piece juxtaposition or the narrow-jam or wide-jam accumulation modes. The wide-jam mode is taken to be governed by the ice force balance equation proposed by Pariset et al. Lal and Shen did recognize that as progression (by shoving) is taking place, there is a simultaneous change in the flow hydraulics. They take care of flow changes by solving the equilibrium thickness and step-backwater equations simultaneously in the reach where the jam is thickening. The RICE model has been used successfully in simulations of ice conditions on the St. Lawrence, Niagara, Ohio, and Yellow rivers, though, like other models, it requires significant calibration to match field data.

Tsai et al. (1988) developed a jam model to investigate ice transport in rivers and ice jam initiation. They used a one-dimensional numerical scheme for solving the ice transport equations, i.e., conservation of ice momentum, ice mass, and ice area. The equations are solved in a Lagrangian form, where the trajectories of ice elements at fixed Eulerian grid points at the beginning of a time step are traced on the $x-t$ plane. Values of ice variables are then interpolated back to the grid points at the end of the time step. The de Saint Venant equations for unsteady water flow are solved using a four-point implicit finite-difference scheme. The ice transport and water flow equations are loosely coupled by first solving the water flow equations and then the ice transport equations based on the new values of the water flow variables.

Shen et al. (1990) elaborated further aspects of this model, examining the various plausible constitutive relationships possible for describing the internal stresses and bank shear. For example, they describe a rapid flow regime as one in which the ice concentration is low and interaction between ice particles is minimal. Commensurately, they characterize a slow flow regime as one in which higher (multi-layer) ice concentrations typically form, and where internal resistance is attributable to prolonged interaction of contacting particles. Their expressions for the streamwise stress $\sigma_{x}$ and the stress normal to the bank $\tau_{x y}$ are equivalent to those for passive pressure resistance, as described by Pariset et al. The authors state the model appears to adequately describe the time and location of jam initiation in river channels, but that more research is necessary to improve the constitutive laws.

\section{Summary}

While considerable progress has been made in modeling the unsteady flow associated with stationary ice jams, the unsteady aspects of ice movement have not been adequately addressed. Most models treat shoving and thickening as an instantaneous phenomenon, with no consideration for the effects of ice momentum on the resulting jam thickness and profile. Physics and field observations suggest that ice momentum should substantially affect jam thickness.

The following sections describe laboratory and numerical experiments aimed at 
evaluating the effects of ice momentum on jam thickness and profile. The numerical model used for this purpose is a significant advance on prior models in so far that it includes ice momentum and directly couples ice and water motion.

\section{LABORATORY EXPERIMENTS}

\section{Introduction}

As indicated in the last section, the literature on ice jams contains no studies describing how jam shoving and thickening occur, or generally evaluating the importance of ice momentum in jam development. The laboratory experiments conducted here provide the first diagnostic information demonstrating the importance of ice momentum.

All prior studies, certainly those that do not include ice motion, treat shoving and thickening as an instantaneous process. When the forces exerted in the downstream direction on a jam reach the level of the passive pressure resistance of the jam, prior formulations let the jam simply thicken. No mention is made of the time required for thickening to take place, or where the ice mass required for the thickening originates. Equilibrium thickness theory (e.g., see Uzuner and Kennedy 1976) carries with it many assumptions, including steady, uniform flow and a stationary ice cover. Certainly, when a jam fails, it violates the latter assumption, which in turn violates the steady and uniform flow condition, because ice movement influences water flow. Once an ice jam comes into motion, the shear stress on the underside of the jam is reduced, because it is a function of the difference between water and ice velocities. Furthermore, the principal assumption used for describing the compressive stress state of ice jams diminishes in validity once a jam fails. The Mohr-Coulomb theory has been used with great success in describing the compressive strength of granular materials, such as ice rubble in a jam, under various states of stress. Once failure begins, however, the material undergoes changes in stress levels that are not well handled using this theory. As well as thickness and velocity changes, other jam characteristics, such as porosity or even ice-piece size or shape, may change.

To model shoving and thickening, the principal effect of ice momentum, it is necessary to know how the process occurs. Though numerous ice jams and their failures have been observed for a wide variety of situations in the field, observations are typically limited to the surface of the cover from the perspective of the shoreline. Even when jam failure and reformation are observed from the air, practical limitations (i.e., altitude and sight distance) render the observations reachaveraged at best. The highly unsteady nature of most breakup jams reduces opportunities for direct measurements of jam properties. Only in the rare incidence where a jam formed and refroze in place, following a reduction in water flow and a return of lower air temperatures, might this be done. While these few cases may provide useful data on jam thickness profiles, other items of interest, such as ice velocity and local water discharge at the time of jamming, remain unknown. A final note concerning jam observations is that, while the date of breakup ice runs and jamming in the northern U.S. might average 10 March (near equal amounts of daylight and darkness daily), about $80 \%$ of ice runs and jams take place during darkness.

To qualitatively examine the importance of ice momentum on jam processes, a laboratory study was undertaken to simulate the shoving and thickening of a failing ice cover. Of particular interest are the timing and mechanics of the process. 
The study used a laboratory flume and a jam formed of ice pieces and plastic beads. The jam was destabilized by means of flow increases. The qualitative observations provided the insights necessary to numerically model unsteady jam formation. A brief series of quantitative experiments was also undertaken to address the applicability of equilibrium thickness theory for determining jam thickness, following a shoving and thickening event.

\section{Experimental setup}

The experiments were undertaken in three different flumes using real and plastic ice. Two of the flumes are refrigerated. One flume has a fixed slope bed $\left(S_{\mathrm{O}}=\right.$ 0.0033 ), is $1.22 \mathrm{~m}$ wide with a working depth of $0.61 \mathrm{~m}$, and is $22.9 \mathrm{~m}$ long. The second flume is tiltable, $36.6 \mathrm{~m}$ long with a working section of $1.22 \mathrm{~m}$ wide by 0.61 $\mathrm{m}$ deep (Fig. 15). Both flumes are housed in refrigerated rooms in which air temperatures can be regulated to $-23^{\circ} \mathrm{C}$. A selection of pumps delivers water from large sumps to each flume, resulting in non-recirculating flow. The sumps contain chiller coils to further reduce water temperature. The quantitative experiments were conducted using the tiltable flume and a third, unrefrigerated flume. This latter flume has a fixed horizontal bed, is $7.3 \mathrm{~m}$ long, and has a working section that is $0.92 \mathrm{~m}$ wide by $0.92 \mathrm{~m}$ deep. It has a large sump and can be operated in either the fully recirculating or "once through" mode.

The visualization experiments entailed initially forming ice covers made of a single layer of ice or plastic beads. Then, water discharge was increased to destabilize the cover and induce shoving and thickening. For the experiments with real ice, ice pieces were formed from a thin sheet (about $15 \mathrm{~mm}$ ) grown in the flume at very low flow. The randomly shaped pieces were approximately $80-120 \mathrm{~mm}$ in their longest dimension. Air temperature was increased to about $0^{\circ} \mathrm{C}$, and the flow was increased until the ice pieces collected as a single-layer accumulation, or jam, held in place by a screen at the downstream end of the flume. The flow rate was

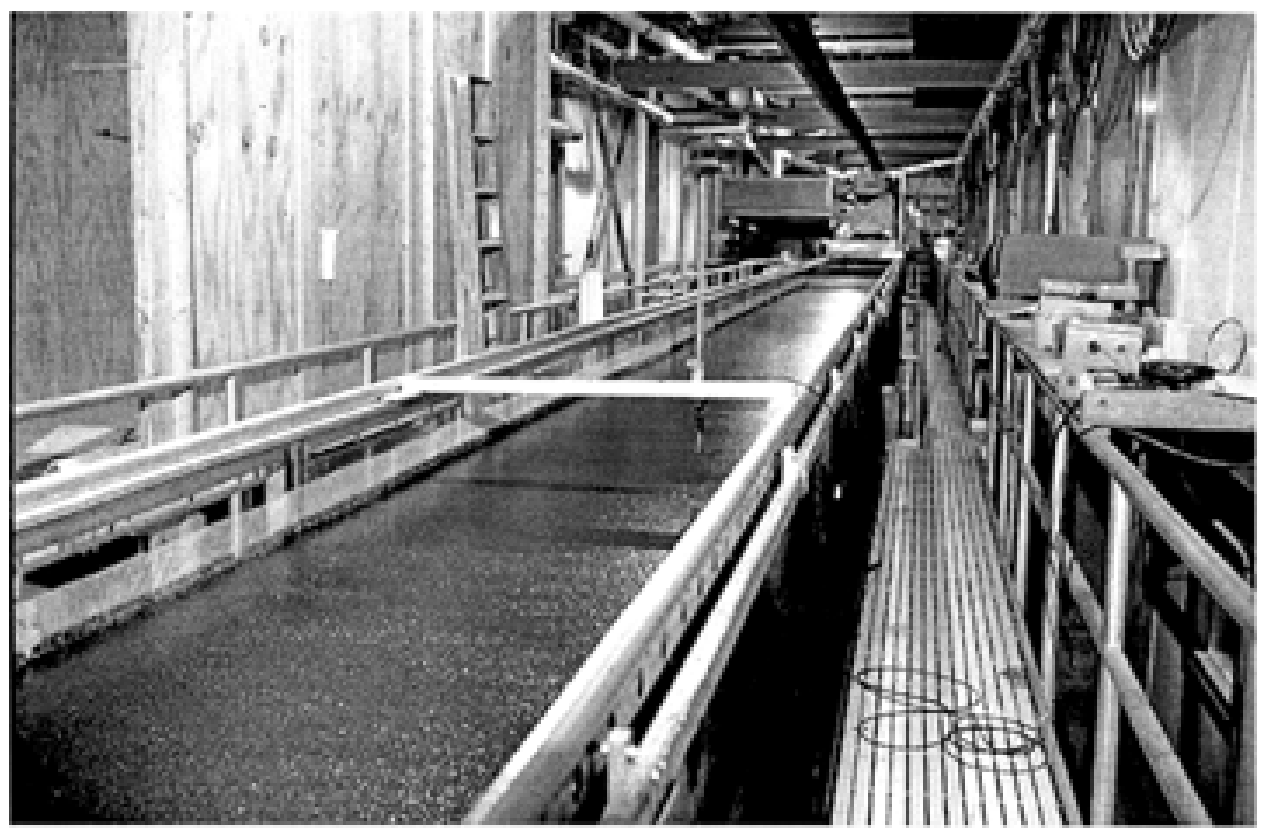

Figure 15. CRREL refrigerated, tiltable flume. 


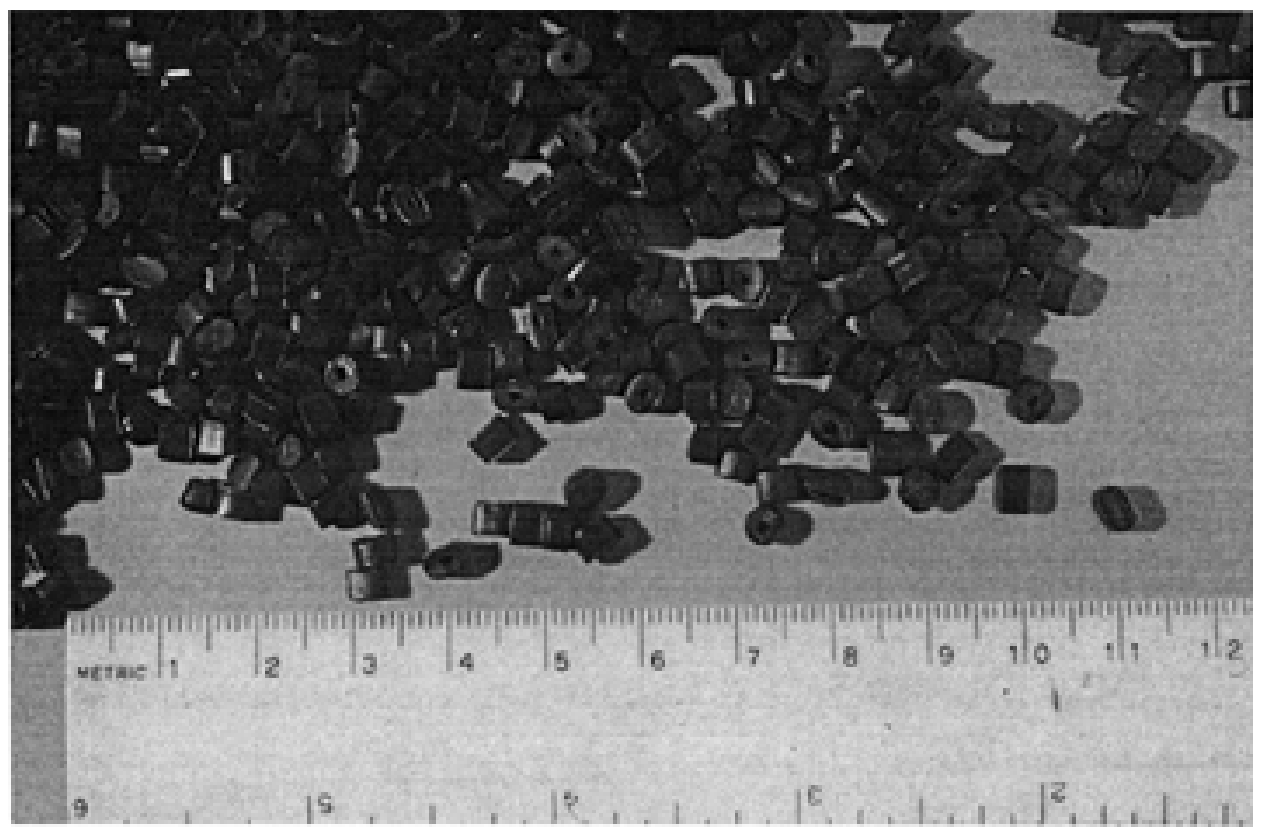

Figure 16. Plastic beads used to simulate ice.

then further increased in steps until the jam destabilized and shoving and thickening took place. Step increases in flow rate were varied from 10 to $100 \%$ of the initial flow rate.

Tests were also conducted using plastic beads formed from extruded polyethylene strands that are chopped to produce uniform pieces approximately cylindrical in shape with a length and diameter of $3 \mathrm{~mm}$ (Fig. 16). The specific gravity of the plastic is 0.925 . The beads form accumulations with a porosity of 0.40 , very similar to natural ice, and have a dry angle of repose of $36^{\circ}$. When new beads are added to the water, they exhibit some surface tension effects (nonwetting), but after a few days they become fully wetted. The beads were used for the visualization experiments in the tiltable flume, as well as for the quantitative testing, because of their constant and uniform properties, as opposed to real ice. For the visualization tests, a uniform layer of beads was spread over the water surface at a very low flow. The flow rate was then increased to the starting flow level for the test and the bead cover was allowed to consolidate, typically resulting in thicknesses of one to two beads. Then, similar to the real ice tests, the flow rate was increased in steps until shoving and thickening took place.

The quantitative experiments were conducted in two series of tests. The first were conducted in the unrefrigerated horizontal-bed flume to determine important physical parameters characterizing accumulations of beads. In these experiments, a stable bead jam was formed at a variety of flow rates, with detailed measurements taken of water surface slopes, depths, accumulation thicknesses, and velocity profiles. From these measurements, the values of the composite, bed, and jam underside friction factors were calculated, as was the value of $\mu$ for the beads. The second series of tests was conducted using the tiltable-bed flume in a warm environment. Similar to the visualization experiments, a bead jam was formed and disturbed by flow-rate increases. Measurements were made of jam thickness, extent, water velocity, water surface slope, and depth. These measurements, together with the values determined for $\mu$ and friction factors, allowed the jam 
thickness, following shoving and thickening, to be compared with that predicted by equilibrium thickness theory.

\section{Observations of shoving and thickening}

The jams failed in two general ways with both the real ice and plastic beads simulating ice. The type of failure was related to the initial flow rate, jam thickness, and the relative increase in flow rate over the initial value.

The first mode of jam failure is called here "progressive jam failure." It was the dominant mode for low initial water discharge, relative to the discharge needed to fail the entire cover, and when the relative increase in discharge was less than $50 \%$. As the discharge was increased, the water level rose first at the upstream end of the flume as the discharge wave traveled its length. The consequent increase in shear stress on the underside of the cover caused minor consolidation at the upstream end of the accumulation and some underturning and transport of individual pieces.

Small ridges of local thickening formed near the jam's upstream end. The ridges developed rather slowly, with the jam upstream moving into the ridge, while the portion of the jam downstream remained motionless. The increased thickness and roughness of the ridge further increased shear stress on the jam's underside in the vicinity of the ridge, initiating additional small failures and ridge-building events further downstream. When new ridges formed downstream, the activity at the upstream ridges slowed or ceased and the entire jam above the most downstream ridge began moving.

With time, this progression of ridge-forming, herein termed the "shoving front," advanced to the jam's downstream end at the screen. At that point, the entire jam was in motion. The jam thickness subsequently increased at the screen until the thickening jam's strength could resist the downstream-acting forces. Although ridgebuilding resulted in minor local thickening as it progressed downstream, the major thickening occurred at the screen, then progressed back upstream. As thickening progressed upstream, the portion of the jam downstream of the "thickening front" became stationary.

Meanwhile, the jam upstream was still moving. Figure 17 provides an idealized picture of the movements of the shoving front and the thickening front during the progressive failure of a jam. Ice velocities during jam failure and thickening were very much less than the bulk water velocity.

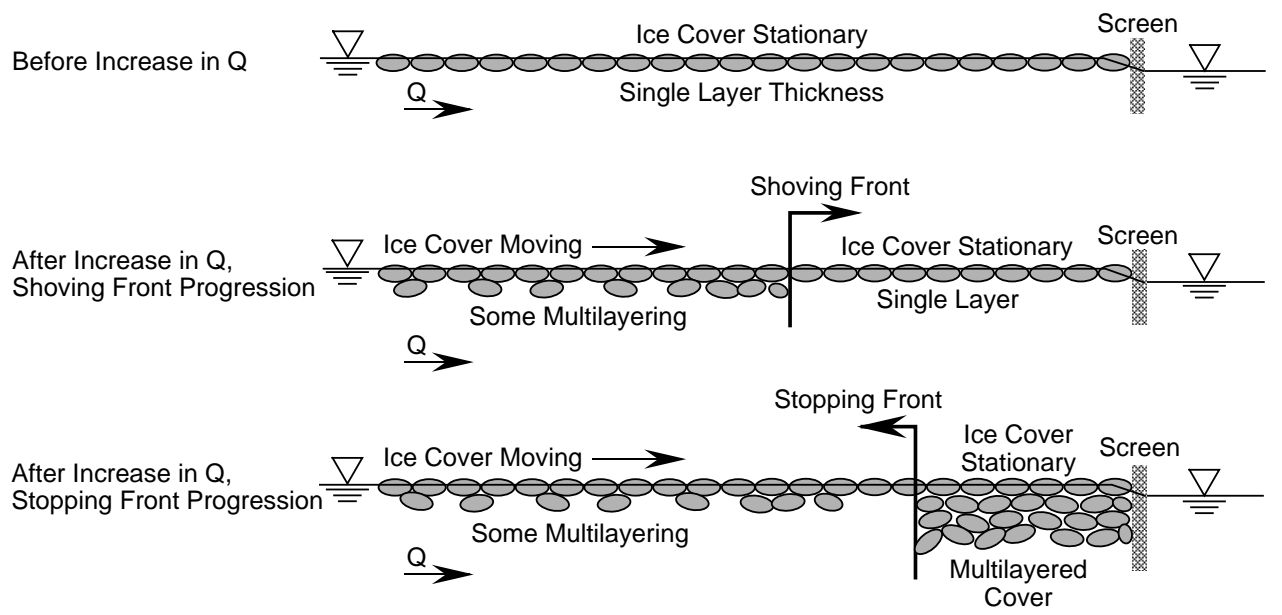

Figure 17. Progressive jam failure. 
The second mode of jam failure is called here "complete jam failure." It was the dominant mode for relatively high initial discharge, for which the entire jam was close to a condition of instability. Discharge increases simply overwhelmed the entire jam. As discharge increased, water level rose, and a wave of water traveled the length of the flume. In these events, however, the entire ice jam (which was up to $30 \mathrm{~m}$ long) mobilized en masse and failed at the downstream screen. The thickening front then progressed upstream from the screen. During some tests with very large discharge increases, small ridges formed elsewhere within the jam, but the major thickening took place as the thickening front swept back upstream. The ice velocities for this type of failure were noticeably higher than for the progressive failure type, yet still only ranged up to $25 \%$ of the bulk water velocity.

A few tests were also conducted in which the discharge was increased and held constant for a short period. These tests were conducted for initial discharges and discharge increases previously identified as causing a progressive jam failure. In these tests, the shoving front was allowed to progress about halfway down the flume, then the discharge was reduced to its original rate. As the discharge receded, the entire jam (which was moving upstream of the shoving front) stopped en masse. This left an ice accumulation that was nearly a single layer thick in the downstream reaches, but slightly thicker upstream. The discharge was then increased to the higher value. In all cases, the jam upstream of where the shoving front had previously progressed mobilized en masse. The shoving front then continued its progression downstream as if the drop and subsequent increase in discharge had never happened.

While the failure modes observed for the ice pieces and plastic beads were generally similar, there were a few differences. As expected, the real ice was more angular and thus had a higher angle of internal resistance. Consequently, the accumulations of real ice were more resistant to increases in downstream load. A complication for the tests using ice was water-temperature regulation. Depending on air temperature, an accumulation may melt or it may further increase its strength owing to freeze-bonding of contacting pieces. The ice pieces were also much larger than the plastic beads, potentially violating assumptions that their behavior could be treated using continuum or particulate theory.

An interesting finding from the tests was that small increases in discharge do not necessarily result in shoving and thickening. Sometimes, two or three small steps in discharge were required to destabilize a jam. This was especially true for the tests using ice, which involved a single layer of ice pieces with a rather high piece aspect ratio $\left(L / \eta_{\mathrm{o}}\right)$, whereas the bead experiments involved a layer thickness of between one and two bead diameters. The high aspect ratio for the ice pieces invalidates assumptions of continuum theory for treating jam strength behavior. The initial discharge used for a test likely was substantially less than the discharge needed to destabilize the jam. For each bead experiment, once a shoving and thickening event was completed, the water discharge was further increased to start a second shoving and thickening event. Usually, multiple discharge steps were required for this, especially when the initial event had led to complete jam failure. Evidently, the collapsed jam had thickened to an extent much greater than the equilibrium thickness estimated from the existing formulations (e.g., Uzuner and Kennedy 1976, Beltaos 1983). This preliminary finding strongly suggests the importance of ice momentum in determining jam thickness. It is this finding that prompted further experiments to compare thicknesses after shoving to those calculated using equilibrium theory. 


\section{Equilibrium thickness evaluations}

To compare thicknesses after failure and thicknesses predicted from equilibrium theory, the strength and hydraulic roughness properties of bead accumulations had to be determined. A useful formulation of static equilibrium thickness is

$$
\eta_{\text {eq }}=\frac{B S}{2 \mu\left(1-s_{\mathrm{i}}\right)}\left\{1+\left[1+\frac{f_{\mathrm{i}} u^{2} \mu\left(1-s_{\mathrm{i}}\right)}{2 B S^{2} s_{\mathrm{i}} g}\right]^{1 / 2}\right\}
$$

in which $u$ is average velocity and $f_{\mathrm{i}}$ is the Darcy-Weisbach friction factor for the flow along the ice cover underside. The overall strength coefficient for the ice $\mu$ is a combination of several material properties, i.e.

$$
\mu=k_{0} \lambda K_{\mathrm{p}}(1-p)
$$

where

$k_{0}=$ lateral stress coefficient (the fraction of the longitudinal acting force that is directed normal to the banks)

$\lambda=$ friction coefficient for ice sliding against ice at a shear boundary

$p=$ accumulation porosity

$K_{\mathrm{p}}=$ Rankine passive pressure coefficient

$$
K_{\mathrm{p}}=\tan ^{2}\left(\frac{\pi}{4}+\frac{\phi}{2}\right)
$$

with $\phi$ being the angle of internal resistance, which is commonly assumed to be equal to the dry angle of repose of a granular material.

Calculation of equilibrium thickness using eq 26 requires knowledge about the average velocity and energy slope of the flow, as well as about the specific gravity of the ice, accumulation porosity, friction factor for flow along the accumulation, and the overall strength coefficient $\mu$. For jam equilibrium, bed slope, water surface slope, and energy slope are taken as being equal. While values of angle of internal resistance $\phi$ and porosity $p$ had been directly measured for the beads, values of lateral stress coefficient $k_{0}$ or the friction coefficient $\lambda$ are unknown. Therefore, $\mu$ cannot be readily calculated from eq 27 . Instead, values were back-calculated using eq 26, but this approach involves $f_{\mathrm{i}}$ as an additional unknown. However, the DarcyWeisbach definition of friction slope for the ice-affected layer of flow

$$
S_{\mathrm{f}}=\frac{f_{\mathrm{i}} u^{2}}{8 g R_{\mathrm{i}}}
$$

combined with eq 26 leads to an alternate form of eq 26

$$
\eta_{\text {eq }}=\frac{B S}{2 \mu\left(1-s_{\mathrm{i}}\right)}\left\{1+\left[1+\frac{4 R_{\mathrm{i}} \mu\left(1-s_{\mathrm{i}}\right)}{s_{\mathrm{i}} B S}\right]^{1 / 2}\right\} .
$$

For this equation, only values of slope and hydraulic radius of the ice-affected flow area are needed to evaluate $\eta_{\text {eq. }}$.

Multi-layer bead accumulations, or jams, were allowed to form in the flume at different levels of steady discharge. Detailed slope measurements and velocity profiles were obtained in the region where the accumulation thickness appeared uniform. Figure 18 presents an example of a measured velocity profile and the fitted 


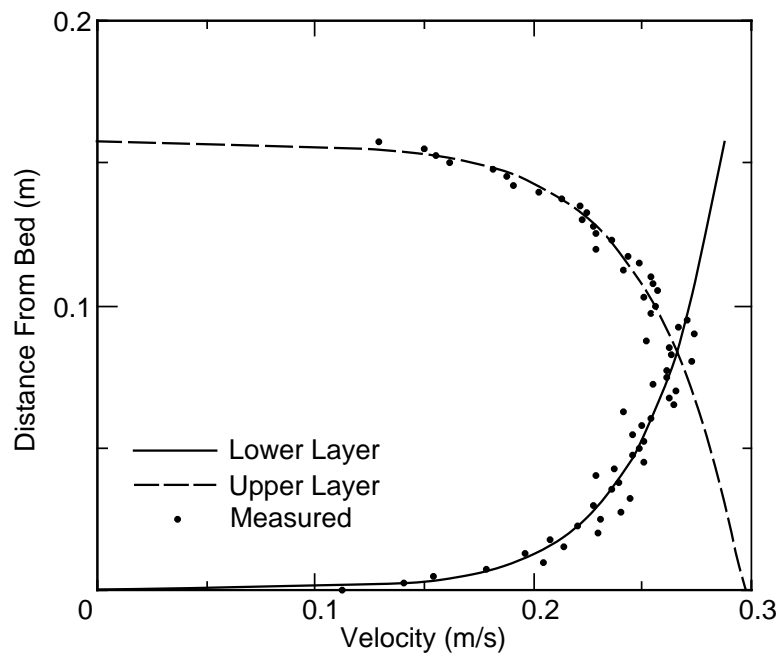

Figure 18. Measured velocity profile with fitted log-law equations for the ice and bed-affected areas.

log-law profiles calculated for the bed- and ice-affected portions of the flow area. By assuming the line of zero shear stress to be equivalent to the intersection of the two computed profiles, the depth of the ice-affected flow area could be determined and the hydraulic radius $R_{\mathrm{i}}$ of that region calculated. The value of $\mu$ was then calculated by back substitution into eq 30 , using the measured value of accumulation thickness in the equilibrium reach. The value of $\mu$ was found to average 0.75 for the three steady flow discharges considered. Though this value is at the low end of the range of 0.8-1.3 reported by Beltaos (1995), it reflects the effect of the difference between the shape of the beads and natural ice rubble. The beads are uniform in size and approximately cylindrical in shape, thereby having an angle of repose less than that of natural ice. The uniform shape of the beads causes their accumulations to deform more easily under stress and results in a lower $K_{\mathrm{p}}$ and thus $\mu$ value than is the case for natural ice rubble in a jam.

With a known average value of $\mu$ determined for the beads, further experiments could proceed using the tiltable-bed flume without refrigeration. Fifteen experiments were conducted under a variety of initial discharges and discharge increases, as reported by Zufelt (1992). Each experiment was run in a manner similar to the visualization series, for which a bead cover was allowed to form at a low discharge and the flow then increased in steps until a shoving and thickening event took place. The energy slope was assumed to be equivalent to the water surface slope, which was calculated from measurements of water surface elevation along the flume. Slope was plotted against discharge, and a linear relation was obtained for the conditions before and after failure. Though a power relationship is to be expected between slope and discharge, the linear fit was adequate for the limited range of discharges used. The two relations also confirm an increase in jam roughness following failure and thickening, as evidenced by the higher slopes following failure (Fig. 19).

The variations in slope for similar discharges before failure in Figure 19 reflect slight variations in the configuration of the bead jam (thickness, extent, etc.) between successive tests. The slope-discharge relation was used for further calculations. The composite Darcy-Weisbach friction factor $f_{\mathrm{o}}$ was calculated for each experiment as

$$
f_{\mathrm{o}}=\frac{8 g R S}{u^{2}}
$$




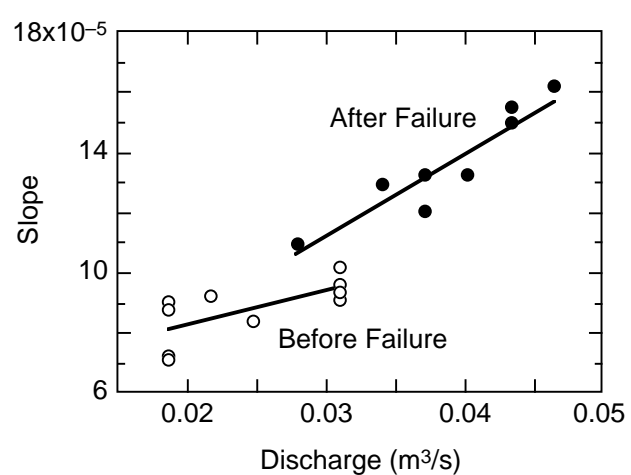

Figure 19. Slope vs. discharge, showing increase in slope after jam failure.

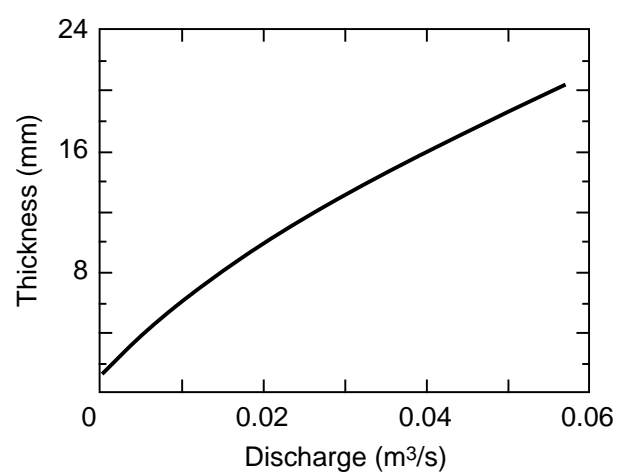

Figure 20. Calculated equilibrium jam thickness vs. discharge.

in which $u$ is average flow velocity and $R$ is the composite or total hydraulic radius. An average value of $f_{\mathrm{o}}$ was calculated and used for the conditions before and after failure. The detailed velocity profiles from each test provided data on the ratio of the bed-affected to ice-affected hydraulic radii. Then, using the Sabaneev equations

$$
f_{\mathrm{o}}=\frac{f_{\mathrm{i}}+f_{\mathrm{b}}}{2}
$$

and

$$
\frac{R_{\mathrm{i}}}{R_{\mathrm{b}}}=\frac{f_{\mathrm{i}}}{f_{\mathrm{b}}}
$$

it is possible to determine ratios of $f_{\mathrm{i}}$ to $f_{\mathrm{b}}$ and $f_{\mathrm{i}}$ to $f_{\mathrm{o}}$. Average values of these ratios for the conditions before and after failure were calculated and used to calculate the equilibrium cover thickness expected for each experiment. Figure 20 is a plot of the equilibrium thickness vs. discharge for this series of experiments, using average values of $f_{\mathrm{o}}$ and $f_{\mathrm{i}} / f_{\mathrm{o}}$.

Figure 21 follows the changes in thickness experienced with step changes in discharge for one of the experiments. The initial thickness of a bead jam is slightly greater than that predicted using the equilibrium theory (eq 30) and was between one and two beads thick. Two step increases in discharge were necessary before a shoving and thickening event occurred, with the resulting thickness again slightly greater than that expected by theory. This first failure was a progressive jam failure with very small ice velocities. Two more step increases in discharge were necessary to again cause jam failure. The second failure was a complete jam failure, with the whole cover mobilizing en masse and thickening taking place initially at the downstream screen. The final thickness was significantly greater than the equilibrium value for that flow level.

The jam failures in each experiment were identified as either progressive or complete jam failures. The final thickness following failure was plotted in Figure 22 against the equilibrium thickness predicted using eq 30 . The progressive jam failures resulted in accumulation thicknesses that plotted on or very slightly above the equality line in the figure. The complete jam failures, however, exceeded the equilibrium thickness in every case, often by a significant amount. 


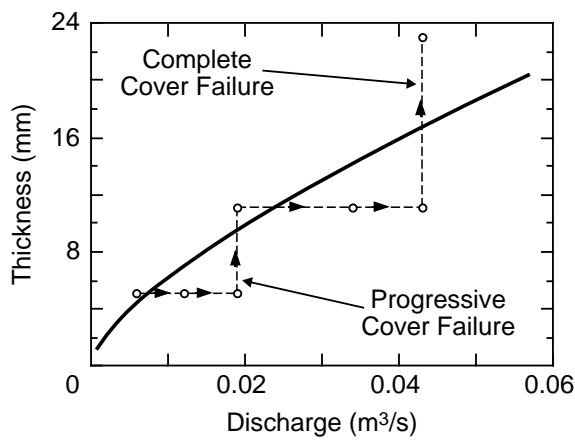

Figure 21. Change in jam thickness with step increases in discharge. A progressive and a complete cover failure are shown.

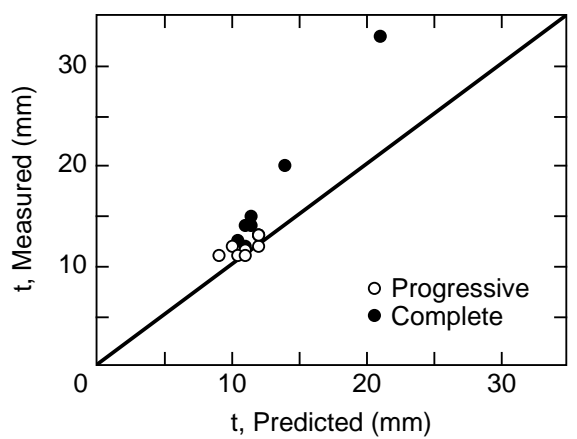

Figure 22. Measured vs. predicted jam thickness following progressive and complete jam failures.

\section{Discussion}

The laboratory experiments were conducted for two reasons: to obtain preliminary insights into the shoving and thickening process, and to examine the applicability of equilibrium thickness theory for determining jam thickness in unsteady water and ice flow situations. The degree of ice movement and its effects on the water flow appeared to depend not only on the material properties of the particulate material composing the jam, but also on the initial discharge and subsequent discharge increase. Two failure modes were identified: progressive and complete jam failure. Progressive jam failure took place at lower initial discharge and lower discharge increases relative to the discharge needed to completely destabilize the jam. It can be characterized as the smooth movement of a shoving front that travels downstream through the jam to the downstream end, causing minor consolidation and thickening. On reaching the downstream screen, a thickening front moves back upstream, resulting in a new, greater jam thickness able to withstand the higher discharge rate. For progressive jam failures, the final jam thickness is very close to, yet slightly greater than, the thickness predicted using equilibrium jam theory as represented in eq 30.

Complete jam failure, on the other hand, occurred for initial discharges close to the discharge necessary to completely destabilize the cover. It can be characterized by the absence of (or instantaneous passage of) the shoving front. The entire jam is moved downstream en masse, failing and thickening at the downstream screen. The final thickness of jam is greater, sometimes significantly so, than the equilibrium thickness predicted using eq 30 .

The experiments, particularly those producing complete jam failure, point to the importance of ice momentum in determining the thickness resulting from the arrest of a moving ice jam. The experiments also indicate that, especially for the progressive jam failures, the time for a shoving and thickening event to occur can be quite significant since the ice velocities are quite low. Shoving and thickening are not instantaneous and should probably not be treated as such. While the progressive jam failure mode may not result in significantly greater ice thickness than that predicted using equilibrium jam theory, there arise conditions of nonuniformity of ice jam thickness, velocity, and depth that could significantly alter the very forces that determine water and ice flow.

Additionally, the experiments reveal the important interactions of ice movement and water flow on one another. They show, therefore, the necessity of using a fully 
coupled numerical model of ice jam formation. Such a model includes both ice velocity and the effects of ice momentum on the force balance, utilizing the full conservation of mass and momentum equations for the ice.

\section{FORMULATION}

\section{Introduction and assumptions}

Formulated here are the one-dimensional, unsteady flow equations for water and ice. The equations, derived in integral form, are based on the conservation of mass and momentum for water and ice flow during jam formation and breakup. The integral equations are then discretized as finite-difference equations, approximating the conservation laws in their integral form. The equations are expressed in terms of four dependent variables that fully describe the flow, as shown in Figure 23 , namely the velocities of the ice cover and under-ice water flow ( $v$ and $u$, respectively), the ice cover thickness $(\eta)$, and the under-ice water depth $(d)$. All four variables are functions of time and space. The equations are derived first in a general form, and then simplified in accordance with the assumptions listed below. Additional simplifications for certain flow conditions are discussed subsequently.

The assumptions made in developing the equations for water and ice flow include the usual St. Venant assumptions for one-dimensional flows (e.g., Cunge et al. 1980) are as follows:

- Flow is one-dimensional, with velocity uniform across each cross section, and water level horizontal at each cross section.

- Streamline curvature is small and vertical accelerations are negligible, so that pressure distribution is hydrostatic.

- The effects of turbulence and boundary friction can be accounted for through resistance laws identical to those used for steady-state flow.

- Average channel-bed slope is small so that the cosine of the angle it makes with the horizontal may be taken as unity. (This assumption is valid for bed slopes to about 0.01.)

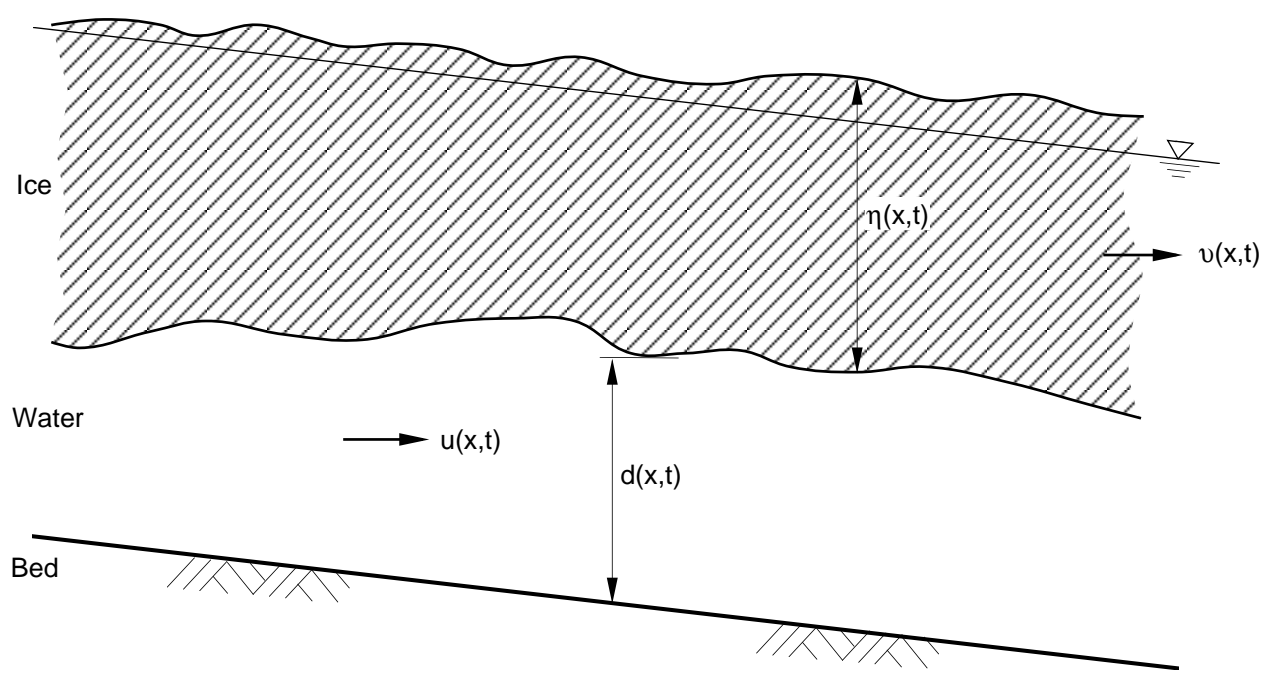

Figure 23. Ice and water flow. 
Other assumptions include:

- Cross sections are uniform and prismatic in shape, ensuring that streamline curvature remains small. For this analysis, a rectangular channel shape is assumed.

- All flow is subcritical. Though there may be considerable changes in depth and ice thickness between two cross sections (notably at the locations of shoving or thickening fronts), it is assumed that through methods (i.e., with no explicit representation of fronts) suitably describe these fronts.

- Ice-piece properties remain constant (i.e., no heat transfer, phase change, or freeze-bonding between ice pieces).

- Jams are particulate continua, such that forces and stresses are describable using Mohr-Coulomb stress theory and an average value across the cross section.

- Jams float with a constant bulk specific gravity, do not ground on the channel bed, and are not subject to significant motion or accelerations in the vertical direction.

\section{Development of equations}

The integral form of the equations for water and ice flow are developed using a control volume approach. The Cartesian coordinate system used is depicted in Figure 24, in which $x$ denotes horizontal distance along the longitudinal river axis, $y$ denotes vertical distance, and $z$ denotes transverse distance normal to the longitudinal axis.

\section{Conservation of water mass}

Conservation of water mass requires the net inflow of water entering a control volume (bounded by $x_{1}, x_{2}$, the bed, and the bottom of the jam in Fig. 24) during a given period be equal to the change in water storage within the control volume for the period, that is

$$
\int_{t_{1}}^{t_{2}}\left[(\rho u A)_{\mathbf{x}_{2}}-(\rho u A)_{\mathbf{x}_{1}}\right] d t+\int_{x_{1}}^{x_{2}}\left[(\rho A)_{\mathbf{t}_{2}}-(\rho A)_{\mathbf{t}_{1}}\right] d x=0 .
$$

For practical purposes, water is incompressible, such that $\rho$ is constant and eq 34 reduces to

$$
\int_{t_{1}}^{t_{2}}\left[(u A)_{\mathbf{x}_{2}}-(u A)_{\mathbf{x}_{1}}\right] d t+\int_{x_{1}}^{x_{2}}\left[(A)_{\mathbf{t}_{2}}-(A)_{\mathbf{t}_{1}}\right] d x=0 .
$$

Further simplifications are made subsequently, such as expressing area $A$ in terms of flow depth $d(x, t)$.

\section{Conservation of ice mass}

The net inflow of ice and pore water (between the ice pieces) into the control volume, bounded by $x_{1}, x_{2}$, and the bottom and top of the jam in Figure 24, is the time integral of the difference between the mass flow rates entering the control volume at $x_{1}$ and leaving the control volume at $x_{2}$, i.e.

$$
\int_{t_{1}}^{t_{2}}\left[\left(\rho_{\mathrm{i}} v A_{\mathrm{i}}[1-p]\right)_{\mathrm{x}_{1}}+\left(\rho v A_{\mathrm{i}} \mathrm{s}_{\mathrm{i}} p\right)_{\mathrm{x}_{1}}-\left(\rho_{\mathrm{i}} v A_{\mathrm{i}}[1-p]\right)_{\mathrm{x}_{2}}-\left(\rho v A_{\mathrm{i}} s_{\mathrm{i}} p\right)_{\mathrm{x}_{2}}\right] d t
$$




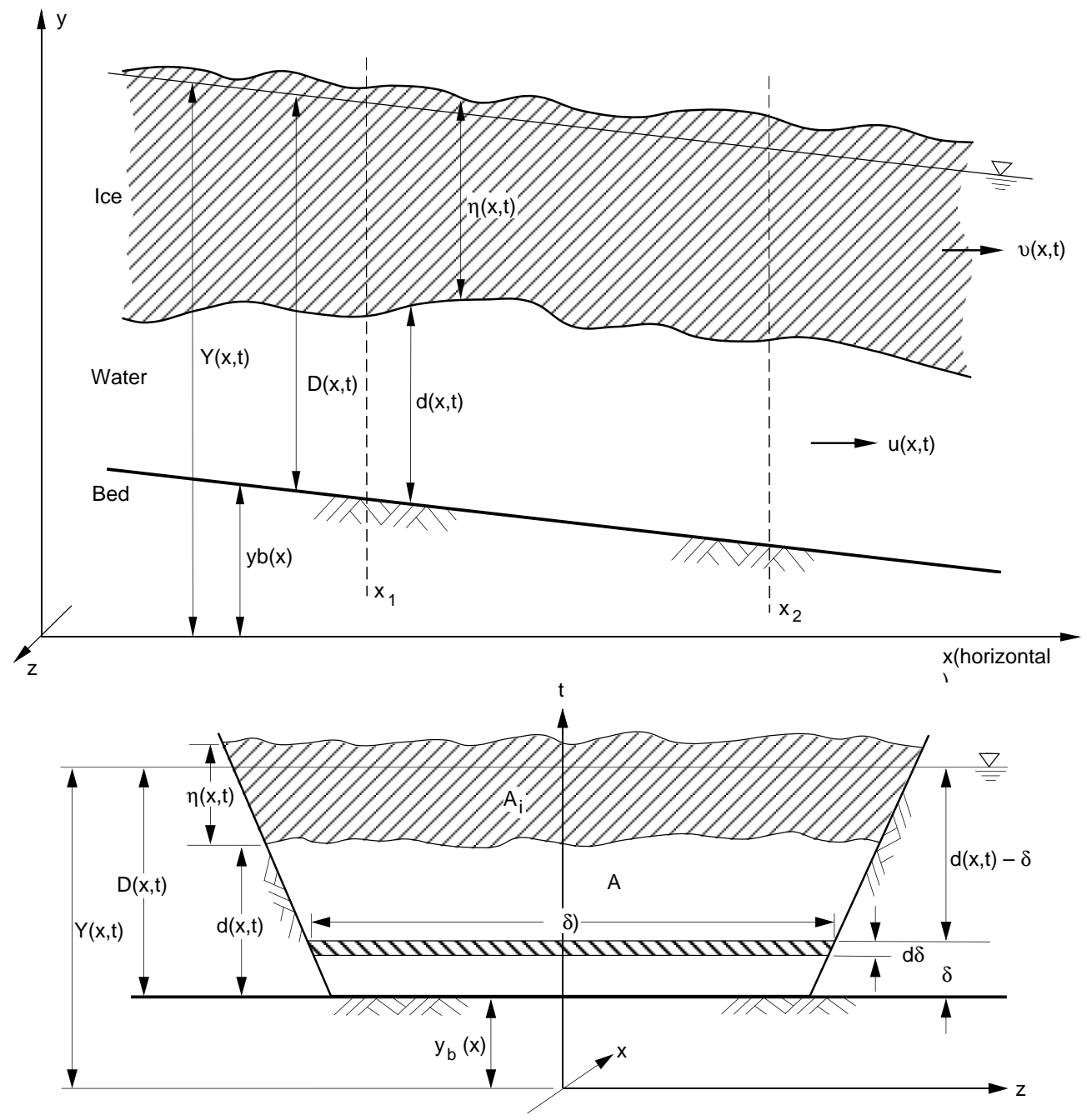

Figure 24. Longitudinal and cross-sectional views of ice and water flow areas, showing coordinate system used in equation development.

where

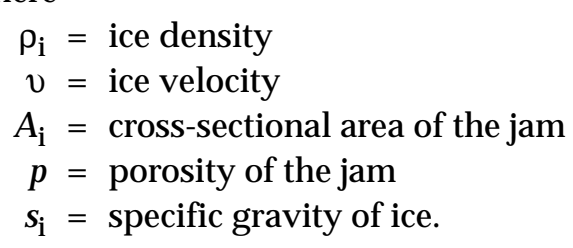

The first and third terms in eq 36 represent the mass flux of ice, while the second and fourth terms represent the pore water. Pore water is only contained in that portion of the ice area below the phreatic surface $\left(s_{\mathrm{i}} A_{\mathrm{i}}\right)$. The experiments of White (1991) show that the velocity of flow through a stationary frazil cover is negligibly small $\left(10^{-5} \mathrm{~m} / \mathrm{s}\right)$, resulting in negligible mass exchange between the pore water and the underlying water flow. Hence, there is no term for seepage flow through the jam provided. Pore water is assumed to move at the same velocity as the ice and since $\rho_{\mathrm{i}}=s_{\mathrm{i}} \rho$, the ice and pore-water terms may be combined. Setting eq 36 
equal to the change in ice storage over the time interval and considering $\rho$ as constant results in

$$
\int_{t_{1}}^{t_{2}}\left[\left(v A_{\mathrm{i}} \mathrm{s}_{\mathrm{i}}\right)_{\mathrm{x}_{2}}-\left(v A_{\mathrm{i}} \mathrm{s}_{\mathrm{i}}\right)_{\mathrm{x}_{1}}\right] d t+\int_{x_{1}}^{x_{2}}\left[\left(A_{\mathrm{i}} \mathrm{s}_{\mathrm{i}}\right)_{\mathrm{t}_{2}}-\left(A_{\mathrm{i}} \mathrm{s}_{\mathrm{i}}\right)_{\mathrm{t}_{1}}\right] d x=0 .
$$

Further simplifications are made subsequently, such as expressing $A_{\mathrm{i}}$ in terms of jam thickness $\eta(x, t)$.

\section{Conservation of water momentum}

The analysis examines the control volume for water flow beneath an ice jam whose channel cross section is prismatic. Figure 25 depicts the forces acting on the control volume that is bounded by $x_{1}, x_{2}$, and the bed and the bottom of the jam.

Conservation of momentum in the $x$-direction requires that the change of momentum within the control volume between times $t_{1}$ and $t_{2}$ equal the sum of the net flux of momentum into the control volume and the integral of the external forces acting on the control volume during the same period. The momentum inside the control volume at any instant is

$$
\int_{x_{1}}^{x_{2}}(\rho A u) d x
$$

so the net increase in momentum $\Delta M$ between times $t_{1}$ and $t_{2}$ is

$$
\Delta M=\int_{x_{1}}^{x_{2}}\left[(\rho A u)_{\mathrm{t}_{2}}-(\rho A u)_{\mathrm{t}_{1}}\right] d x .
$$

The net momentum flux $M_{\mathrm{f}}$ into the control volume between times $t_{1}$ and $t_{2}$ is

$$
M_{\mathrm{f}}=\int_{\mathrm{t}_{1}}^{\mathfrak{t}_{2}}\left[\left(\rho A u^{2}\right)_{\mathrm{x}_{1}}-\left(\rho A u^{2}\right)_{\mathrm{x}_{2}}\right] d t .
$$

The external forces acting on the water control volume include: hydrostatic pressure; gravity forces due to the weight of the water, ice, and pore water; and shear stress at the bed, banks, and jam underside. The hydrostatic pressure forces acting at sections $x_{1}$ and $x_{2}$ are and as depicted in Figure 25. With the level of the phreatic surface above the bed denoted as $D(x)$, the vertical distance above the bed as $\delta(x)$, and the local width as $b(\delta)$, for any section $x$

Figure 25. Forces acting on the water control volume.

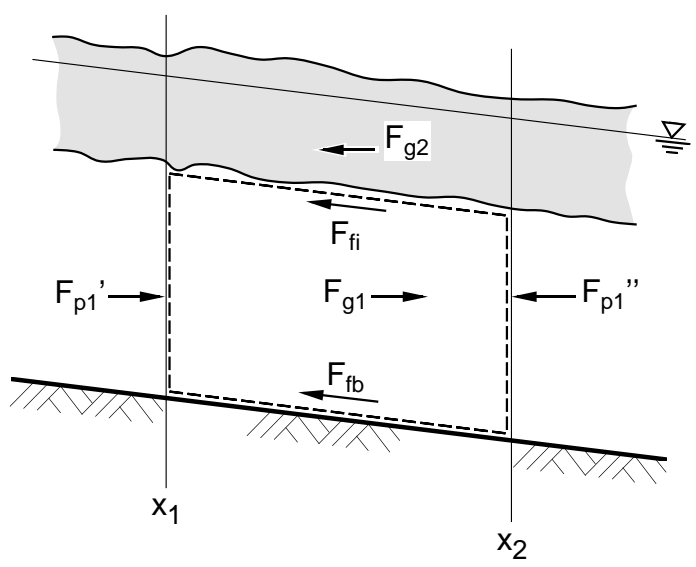




$$
F_{\mathrm{p} 1}{ }^{\prime}=g \int_{0}^{d(x)} \rho[D(x)-\delta] b(x, \delta) d \delta .
$$

For a rectangular channel, $b$ is constant in the vertical and equal to the top width $B$. Therefore

$$
F_{\mathrm{p} 1}^{\prime}=g \int_{0}^{d(x)} \rho[D(x)-\delta] B d \delta .
$$

Substitution of $D(x)=d(x)+s_{\mathrm{i}} \eta(x)$ and integration gives

$$
F_{\mathrm{p} 1}^{\prime}=\rho g B\left[d^{2} / 2+d s_{\mathrm{i}} \eta\right] .
$$

Thus, the time integral for the net pressure $F_{\mathrm{p} 1}$ is

$$
\int_{t_{1}}^{t_{2}} F_{\mathrm{p} 1} d t=\int_{t_{1}}^{t_{2}}\left(F_{\mathrm{p} 1}{ }^{\prime}-F_{\mathrm{p} 1}{ }^{\prime \prime}\right) d t=g \int_{t_{1}}^{t_{2}}\left[\left(\rho I_{1}\right)_{\mathbf{x}_{1}}-\left(\rho I_{1}\right)_{\mathbf{x}_{2}}\right] d t
$$

where

$$
I_{1}=B\left[d^{2} / 2+d s_{\mathrm{i}} \eta\right] .
$$

Two gravity forces act vertically on the water control volume. The first acts on the bottom surface of the control volume (the bed). It is attributable to the combined weight of water, ice, and pore water above. The second acts on the upper surface of the control volume (the bottom of the jam). It is attributable to the weight of the ice and pore water above. The horizontal component of the first gravity force is

$$
F_{\mathrm{g} 1}=\int_{x_{1}}^{x_{2}}\left(\rho g A+\rho_{\mathrm{i}} g A_{\mathrm{i}}(1-p)+\rho g A_{\mathrm{i}} \mathrm{s}_{\mathrm{i}} p\right) S_{\mathrm{o}} d x=\int_{x_{1}}^{x_{2}} \rho g\left(A+A_{\mathrm{i}} \mathrm{s}_{\mathrm{i}}\right) S_{\mathrm{o}} d x
$$

where $S_{\mathrm{O}}$ is bed slope

$$
S_{\mathrm{o}}=-\frac{\partial y_{\mathrm{b}}}{\partial x} .
$$

For the period $t_{1}$ to $t_{2}$

$$
\int_{t_{1}}^{t_{2}} F_{\mathrm{g} 1} d t=\int_{t_{1} x_{1}}^{t_{2} x_{2}} \rho g\left(A+A_{\mathrm{i}} S_{\mathrm{i}}\right) S_{\mathrm{o}} d x d t .
$$

The gravity force attributable to the weight of ice and pore water acting on the upper surface of the control volume (in the $x$-direction) is

$$
F_{\mathrm{g} 2}=\int_{x_{1}}^{x_{2}}\left[\rho_{\mathrm{i}} g A_{\mathrm{i}}(1-p) S_{\mathrm{ib}}+\rho g A_{\mathrm{i}} s_{\mathrm{i}} p S_{\mathrm{ib}}\right] d x=\int_{x_{1}}^{x_{2}}\left[\rho g A_{\mathrm{i}} s_{\mathrm{i}} S_{\mathrm{ib}}\right] d x
$$

where $S_{\mathrm{ib}}$ is the slope of the jam underside

$$
S_{\mathrm{ib}}=-\frac{\partial\left(y_{\mathrm{b}}+d\right)}{\partial x}=-\left[\frac{\partial y_{\mathrm{b}}}{\partial x}+\frac{\partial d}{\partial x}\right]=S_{\mathrm{o}}-\frac{\partial d}{\partial x} .
$$

Substituting eq 50 into 49 and integrating over the period $t_{1}$ to $t_{2}$ gives

$$
\int_{t_{1}}^{t_{2}} F_{\mathrm{g} 2} d t=\int_{t_{1} x_{1}}^{t_{2} x_{2}} \rho g A_{\mathrm{i}} s_{\mathrm{i}}\left(S_{\mathrm{o}}-\frac{\partial d}{\partial x}\right) d x d t .
$$


The remaining forces to be determined are the boundary resistance or shear forces. The total shear stress produced by the water flow (averaged for the control-volume reach) is

$$
\tau=\rho g R S_{\mathrm{f}}
$$

where $R$ is the hydraulic radius of the section and $S_{\mathrm{f}}$ is the friction or energy slope associated with the water flow. The Darcy-Weisbach definition of friction slope is

$$
S_{\mathrm{f}}=\frac{f_{\mathrm{o}} u^{2}}{8 R g}
$$

where $f_{\mathrm{o}}$ is the composite (bed and ice cover) Darcy-Weisbach resistance factor. Equation 53 substituted into eq 52 gives

$$
\tau=\frac{\rho g R f_{\mathrm{o}} u^{2}}{8 R g}=\frac{\rho f_{\mathrm{o}} u^{2}}{8} \text {. }
$$

This shear stress is a total value, generated by the differences in the velocities of water flow relative to the velocities of the other boundaries of the control volume. It can be split into two parts: $\tau_{\mathrm{b}}$, the shear stress at the bed and bank boundary, and $\tau_{i}$, the shear stress at the ice boundary. Prior formulations (e.g., Beltaos 1983) have shown that the simple case of a static ice cover can be analyzed approximately using a "two-layer approach," separating the total flow area into one layer dominated by shear stress on the bed and banks, and another layer dominated by shear

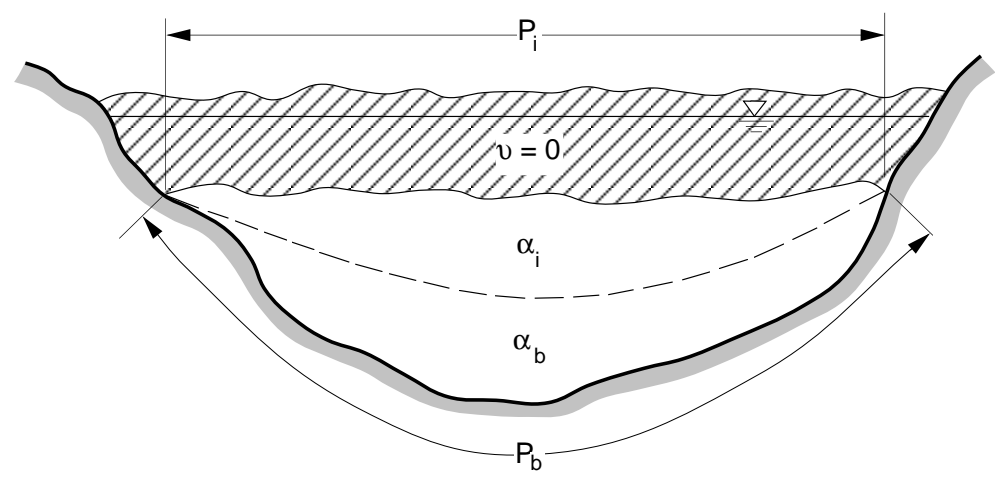

a)

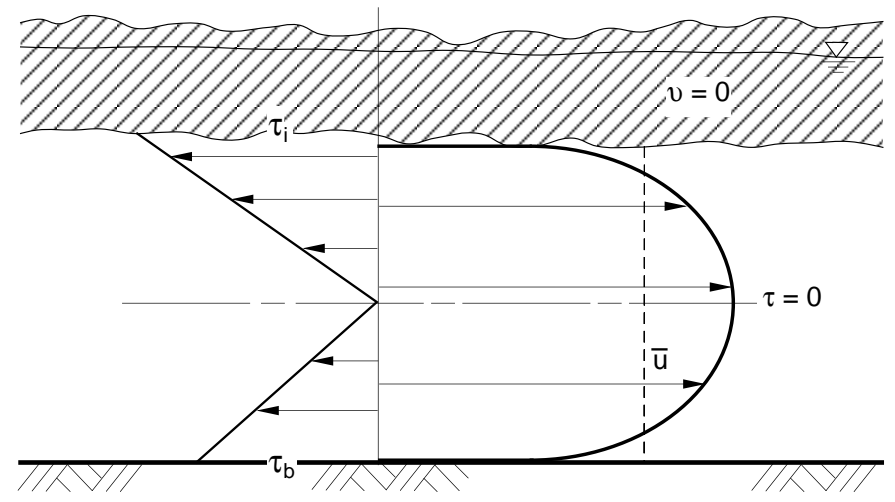

b)

Figure 26. Two-layer approach designation of shear stress due to water flow. 
stress on the ice cover. Figure 26 identifies the two layers. Note that $\alpha$ refers to the area of each respective layer, $P$ is the wetted perimeter, and the subscripts $b$ and $i$ designate the bed-and ice-affected variables. The dashed line indicates the line of nominal zero shear or the boundary between the two layers. The total shear force per unit length of flow area is

$$
\tau P=\tau_{\mathrm{b}} P_{\mathrm{b}}+\tau_{\mathrm{i}} P_{\mathrm{i}}
$$

If the "two-layer approach" is valid for any value of ice velocity $v$, such as depicted in Figure 27, then the shear stress at each boundary is expressible as

$$
\tau_{\mathrm{b}}=\frac{\rho f_{\mathrm{b}} u|u|}{8}
$$

and

$$
\tau_{\mathrm{i}}=\frac{\rho f_{\mathrm{i}}(u-v)|u-v|}{8} .
$$

The absolute value sign captures directional shear. It can be dropped provided that stress direction is preserved in the momentum equation.

The "two-layer approach" assumes that each layer can be adequately described using the Darcy-Weisbach relationship for flow resistance and thus can be related to the friction slope of the water flow, i.e.

$$
S_{\mathrm{f}}=\frac{f_{\mathrm{b}} u^{2}}{8 R_{\mathrm{b}} g}=\frac{f_{\mathrm{i}}(u-v)^{2}}{8 R_{\mathrm{i}} g} .
$$

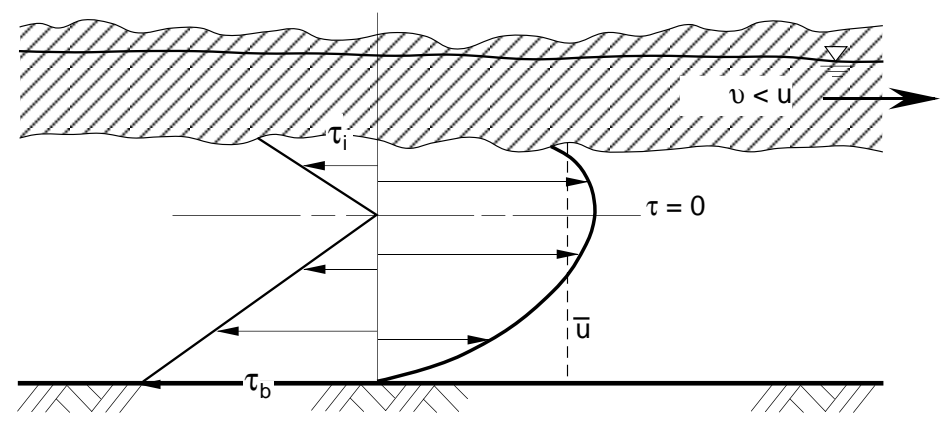

a)

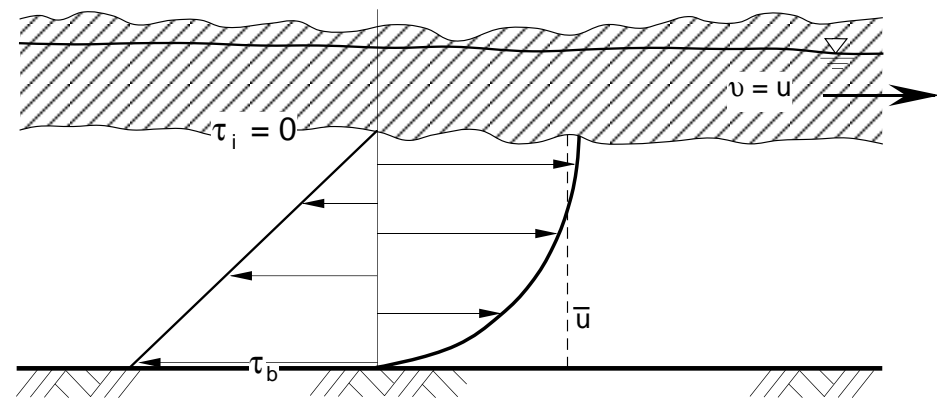

b)

Figure 27. Shear stress due to water flow for cases of a moving jam. 
As $R=A / P$, eq 58 can be rewritten as

$$
\left(\frac{\alpha_{\mathrm{i}}}{\alpha_{\mathrm{b}}}\right)=\left(\frac{f_{\mathrm{i}}}{f_{\mathrm{b}}}\right)\left(\frac{P_{\mathrm{i}}}{P_{\mathrm{b}}}\right)\left(\frac{u-v}{u}\right)^{2}
$$

where $\alpha_{b}$ and $\alpha_{i}$ are the bed and ice-affected portions of the flow area. The total shear stress is $\tau_{\mathrm{b}}+\tau_{\mathrm{i}}$ and the total shear force on a unit length of channel is $\tau_{\mathrm{b}} P_{\mathrm{b}}+$ $\tau_{\mathrm{i}} P_{\mathrm{i}}$, which leads to the following expression for $S_{\mathrm{f}}$

$$
S_{\mathrm{f}}=\frac{\tau}{\rho g R}=\frac{\tau P}{\rho g A}=\frac{\tau_{\mathrm{b}} P_{\mathrm{b}}+\tau_{\mathrm{i}} P_{\mathrm{i}}}{\rho g A} .
$$

The time integral of the friction force is

$$
\int_{t_{1}}^{t_{2}} F_{\mathrm{f}} d t=\int_{t_{1} x_{1}}^{t_{2} x_{2}} \rho g A S_{\mathrm{f}} d x d t=\int_{t_{1} x_{1}}^{t_{2} x_{2}}\left[\tau_{\mathrm{b}} P_{\mathrm{b}}+\tau_{\mathrm{i}} P_{\mathrm{i}}\right] d x d t
$$

Equations 55 to 57 , rearranged in terms of the unknown variables $u, v, d$, and $\eta$, become

$$
\begin{aligned}
& \tau_{\mathrm{b}} P_{\mathrm{b}}+\tau_{\mathrm{i}} P_{\mathrm{i}}=\frac{\rho f_{\mathrm{b}} u^{2} P_{\mathrm{b}}}{8}+\frac{\rho f_{\mathrm{i}}(u-v)^{2} P_{\mathrm{i}}}{8} \\
& =\frac{\rho f_{\mathrm{b}} u^{2} P_{\mathrm{b}}}{8}\left[1+\frac{f_{\mathrm{i}}(u-v)^{2} P_{\mathrm{i}}}{f_{\mathrm{b}} u^{2} P_{\mathrm{b}}}\right]=\frac{\rho f_{\mathrm{b}} u^{2} P_{\mathrm{b}}}{8}\left[1+\frac{\alpha_{\mathrm{i}}}{\alpha_{\mathrm{b}}}\right] .
\end{aligned}
$$

An expression for $S_{\mathrm{f}}$ results when eq 59 and 62 are combined

$$
S_{\mathrm{f}}=\frac{\tau_{\mathrm{b}} P_{\mathrm{b}}+\tau_{\mathrm{i}} P_{\mathrm{i}}}{\rho g A}=\frac{f_{\mathrm{b}} u^{2} P_{\mathrm{b}}}{8 g A}\left[1+\frac{f_{\mathrm{i}}}{f_{\mathrm{b}}} \frac{P_{\mathrm{i}}}{P_{\mathrm{b}}}\left(\frac{u-v}{u}\right)^{2}\right] .
$$

The effects of flow and ice velocities on $\tau_{\mathrm{i}} P_{\mathrm{i}}$ are evident in Figure 28. As ice velocity increases beyond water velocity, the shear stress caused by the jam's presence reverses to the downstream direction and the portion of the flow area affected by this force increases.

The full momentum equation for the water flow can be written as

$$
\Delta M-M_{\mathrm{f}}=\int_{t_{1}}^{t_{2}} F_{\mathrm{p} 1} d t+\int_{t_{1}}^{t_{2}} F_{\mathrm{g} 1} d t-\int_{t_{1}}^{t_{2}} F_{\mathrm{g} 2} d t-\int_{t_{1}}^{t_{2}} F_{\mathrm{f}} d t .
$$

Equations 39, 40, 44, 48, 51, and 61 combined with eq 64 give

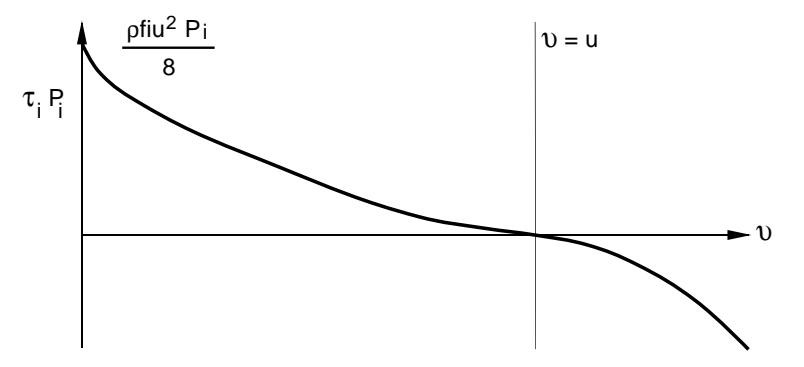

Figure 28. Shear force on the ice jam underside vs. ice velocity $\left(\tau_{i} \mathrm{P}_{i} v s . v\right)$. 


$$
\begin{aligned}
& \int_{x_{1}}^{x_{2}}\left[(\rho u A)_{\mathrm{t}_{2}}-(\rho u A)_{\mathrm{t}_{1}}\right] d x-\int_{t_{1}}^{t_{2}}\left[\left(\rho u^{2} A\right)_{\mathrm{x}_{1}}-\left(\rho u^{2} A\right)_{\mathrm{x}_{2}}\right] d t= \\
& g \int_{t_{1}}^{t_{2}}\left[\left(\rho I_{1}\right)_{\mathrm{x}_{1}}-\left(\rho I_{1}\right)_{\mathrm{x}_{2}}\right] d t+g \iint_{t_{1} x_{1}}^{t_{2} x_{2}} \rho\left(A+A_{\mathrm{i}} s_{\mathrm{i}}\right) S_{\mathrm{o}} d x d t \\
& -g \int_{t_{1} x_{1}}^{t_{2} x_{2}} \rho A_{\mathrm{i}} \mathrm{s}_{\mathrm{i}}\left(S_{\mathrm{o}}-\frac{\partial d}{\partial x}\right) d x d t-g \iint_{t_{1} x_{1}}^{t_{2} x_{2}} \rho A S_{\mathrm{f}} d x d t .
\end{aligned}
$$

If $\rho$ is constant, eq 65 simplifies to

$$
\begin{aligned}
& \int_{x_{1}}^{x_{2}}\left[(u A)_{\mathrm{t}_{2}}-(u A)_{\mathrm{t}_{1}}\right] d x-\int_{t_{1}}^{t_{2}}\left[\left(u^{2} A\right)_{\mathrm{x}_{1}}-\left(u^{2} A\right)_{\mathrm{x}_{2}}\right] d t=g \int_{t_{1}}^{t_{2}}\left[\left(I_{1}\right)_{\mathrm{x}_{1}}-\left(I_{1}\right)_{\mathrm{x}_{2}}\right] d t+ \\
& g \int_{t_{1} x_{1}}^{t_{2} x_{2}} A_{\mathrm{i}} s_{\mathrm{i}} \frac{\partial d}{\partial x} d x d t+g \int_{t_{1} x_{1}}^{t_{2} x_{2}} A\left(S_{\mathrm{o}}-S_{\mathrm{f}}\right) d x d t .
\end{aligned}
$$

\section{Conservation of ice momentum}

The ice control volume under analysis is bounded by $x_{1}, x_{2}$, and the bottom and top of the jam. Figure 29 depicts the control volume and the forces acting on the jam. The force associated with wind drag at the air/ice interface is neglected in this formulation, though it could readily be included. The momentum of the ice and pore water in the control volume at any instant is

$$
\int_{x_{1}}^{x_{2}}\left(\rho_{\mathrm{i}} v A_{\mathrm{i}}[1-p]+\rho v A_{\mathrm{i}} s_{\mathrm{i}} p\right) d x .
$$

From $\rho_{i}=s_{i} \rho$, and regrouping the $p$ and $(1-p)$ terms, the net increase in momentum within the control volume between $t_{1}$ and $t_{2}$ becomes

$$
\Delta M=\int_{x_{1}}^{x_{2}}\left[\left(s_{\mathrm{i}} \rho v A_{\mathrm{i}}\right)_{\mathrm{t}_{2}}-\left(s_{\mathrm{i}} \rho v A_{\mathrm{i}}\right)_{\mathrm{t}_{1}}\right] d x .
$$

The net momentum flux into the control volume is

$$
\left[\left(\rho_{\mathrm{i}} v^{2} A_{\mathrm{i}}[1-p]\right)+\left(\rho v^{2} A_{\mathrm{i}} s_{\mathrm{i}} p\right)\right]_{\mathrm{x}_{1}}-\left[\left(\rho_{\mathrm{i}} v^{2} A_{\mathrm{i}}[1-p]\right)+\left(\rho v^{2} A_{\mathrm{i}} s_{\mathrm{i}} p\right)\right]_{\mathrm{X}_{2}} .
$$

Once again, from $\rho_{i}=s_{i} \rho$, and regrouping the $p$ and $(1-p)$ terms, the net momentum flux into the control volume between $t_{1}$ and $t_{2}$ becomes

$$
M_{\mathrm{f}}=\int_{t_{1}}^{t_{2}}\left[\left(\rho v^{2} A_{\mathrm{i}} s_{\mathrm{i}}\right)_{\mathrm{x}_{1}}-\left(\rho v^{2} A_{\mathrm{i}} \mathrm{s}_{\mathrm{i}}\right)_{\mathrm{x}_{2}}\right] d t
$$

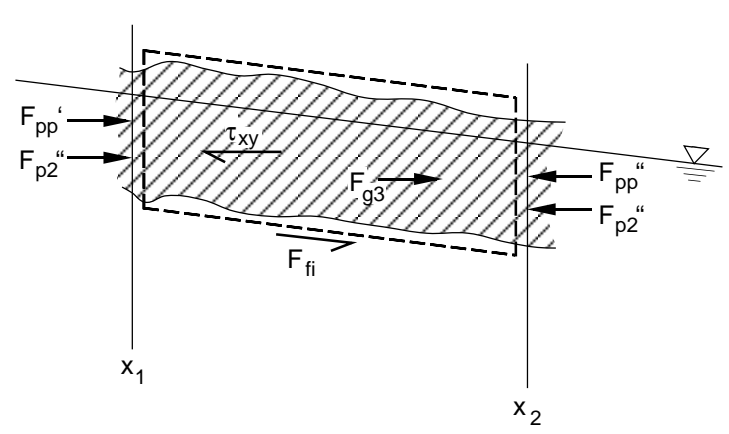

Figure 29. Forces acting on the ice control volume. 
The external forces acting on the ice and pore water control volume include: hydrostatic pressure, a gravity force due to the ice and pore water in $x$-direction, shear stress on the underside of the jam from the water flow, and shear stress at the banks. The strength of the jam also resists ice motion, provided stress acting through the jam does not exceed the maximum allowable longitudinal stress. That limiting stress places the jam in the Rankine passive state of stress.

The hydrostatic pressure forces acting at sections $x_{1}$ and $x_{2}$ are $F_{\mathrm{p} 2}{ }^{\prime}$ and $F_{\mathrm{p} 2}{ }^{\prime \prime}$ respectively. With the level of the phreatic surface above the bottom of the jam denoted as $s_{\mathrm{i}} \eta(x)$, the vertical distance above the bottom of the jam denoted as $\delta(x)$, and the local width as $b(\delta)$, then for any section $x$

$$
F_{\mathrm{p} 2}^{\prime}=g \int_{0}^{s_{\mathrm{i}} \eta(x)} \rho\left[s_{\mathrm{i}} \eta(x)-\delta\right] b(x, \delta) d \delta
$$

For a rectangular channel, $b$ is constant in the vertical and equal to the top width $B$. Thus

$$
F_{\mathrm{p} 2}^{\prime}=g \int_{0}^{s_{\mathrm{i}} \eta(x)} \rho\left[s_{\mathrm{i}} \eta(x)-\delta\right] B d \delta .
$$

Integration of eq 72 yields

$$
F_{\mathrm{p} 2}^{\prime}=\rho g B \frac{\left(s_{\mathrm{i}} \eta\right)^{2}}{2} \text {. }
$$

Consequently, the time integral of the net pressure $F_{\mathrm{p} 2}$ is

$$
\int_{t_{1}}^{t_{2}} F_{\mathrm{p} 2} d t=\int_{t_{1}}^{t_{2}}\left(F_{\mathrm{p} 2}{ }^{\prime}-F_{\mathrm{p} 2}{ }^{\prime \prime}\right) d t=g \int_{t_{1}}^{t_{2}}\left[\left(\rho I_{2}\right)_{\mathrm{x}_{1}}-\left(\rho I_{2}\right)_{\mathrm{x}_{2}}\right] d t
$$

where

$$
I_{2}=B \frac{\left(s_{\mathrm{i}} \eta\right)^{2}}{2}
$$

A gravity force acts on the bottom surface of the ice control volume (the bottom of the jam) from the weight of the ice and pore water above. The horizontal component of this gravity force equals and counterbalances $F_{\mathrm{g} 2}$

$$
F_{\mathrm{g} 3}=\int_{x_{1}}^{x_{2}}\left[\rho_{\mathrm{i}} g A_{\mathrm{i}}(1-p) S_{\mathrm{ib}}+\rho g A_{\mathrm{i}} s_{\mathrm{i}} p S_{\mathrm{ib}}\right] d x=\int_{x_{1}}^{x_{2}}\left[\rho g A_{\mathrm{i}} s_{\mathrm{i}} S_{\mathrm{ib}}\right] d x .
$$

For the period $t_{1}$ to $t_{2}$

$$
\int_{t_{1}}^{t_{2}} F_{\mathrm{g} 3} d t=\int_{t_{1} x_{1}}^{t_{2} x_{2}} \rho g A_{\mathrm{i}} s_{\mathrm{i}}\left(S_{\mathrm{o}}-\frac{\partial d}{\partial x}\right) d x d t
$$

The shear stress on the underside of the jam attributable to water flow is equal and opposite to the shear force of the cover on the water, i.e.

$$
\tau_{\mathrm{i}}=\int_{t_{1}}^{t_{2}} F_{\mathrm{fi}} d t
$$

In the case of the jam, however, only the shear force along the jam underside, or $\tau_{\mathrm{i}} P_{\mathrm{i}}$, is included. From eq 62 above 


$$
\tau_{\mathrm{i}} P_{\mathrm{i}}=\frac{\rho f_{\mathrm{i}}(u-v)^{2} P_{\mathrm{i}}}{8} .
$$

Substituting for $F_{\text {fi }}$ gives

$$
\int_{t_{1}}^{t_{2}} F_{\mathrm{fi}} d t=\int_{t_{1} x_{1}}^{t_{2} x_{2}} \tau_{\mathrm{i}} P_{\mathrm{i}} d x d t=\int_{t_{1} x_{1}}^{t_{2} x_{2}} \frac{\rho f_{\mathrm{i}}(u-v)^{2} P_{\mathrm{i}}}{8} d x d t .
$$

From the assumption that ice jams can be treated as a particulate continuum, the vertical and horizontal stresses within jams can be related using Rankine stress theory. Previous researchers, as discussed in the Review of Ice Jam Modeling section, give the shear stress at the banks $\tau_{x y}$ as a function of the stress along the jam's longitudinal axis $\sigma_{\mathrm{x}}$. For a granular material, the normal stress can be expressed directly as a function of the vertical stress $\sigma_{\mathrm{v}}$, which results from the weight of ice acting downward to the phreatic surface through the jam, and from the buoyancy force acting upward in the submerged portion. Vertical stress ascribable to ice weight acting downward throughout the entire cover thickness is

$$
\sigma_{\mathrm{vi}}=\rho_{\mathrm{i}} g y(1-p)
$$

where $y$ is measured from the top of the ice surface. The stress of the water acting downward in the wetted portion is

$$
\sigma_{\mathrm{vw}}=\rho g\left(y-\left[1-s_{\mathrm{i}}\right] \eta\right) p
$$

where $y$ is measured from the top surface of the ice and $y=\left(1-s_{\mathrm{i}}\right) \eta$ represents the phreatic surface. The buoyancy force acting upward in the wetted portion of the thickness is

$$
u^{\prime}=\rho g\left(y-\left[1-s_{\mathrm{i}}\right] \eta\right)
$$

From eq 81 to 83 , the total or effective vertical stress $\sigma_{\mathrm{v}}$ is zero at the upper and lower surfaces of the jam, and $\sigma_{\mathrm{v}}$ reaches a maximum at the phreatic surface. Over the entire thickness, the average vertical stress is

$$
\overline{\sigma_{\mathrm{v}}}=s_{\mathrm{i}} \rho g(1-p)\left(1-s_{\mathrm{i}}\right) \frac{\eta}{2} \text {. }
$$

The horizontal or longitudinal stress in a granular material relates to the average vertical stress as

$$
\sigma_{\mathrm{x}}=k_{1} \overline{\sigma_{\mathrm{v}}}
$$

where $k_{1}$ is a coefficient of proportionality reflecting the jam's state of stress, i.e., passive, neutral, or active. For passive-pressure loading conditions, $k_{1}$ typically assumes the value of the passive pressure coefficient $K_{p}$, which represents the maximum or failure stress of the material

$$
K_{\mathrm{p}}=\tan ^{2}\left(45+\frac{\phi}{2}\right)
$$

where $\phi$ is the angle of internal resistance measured in degrees. Equations 81, 82, 
83 , and 85 combine to give the force due to normal pressure for a general channel shape, i.e.

$$
\begin{aligned}
& {F_{\mathrm{pp}}}^{\prime}= \\
& K_{\mathrm{p} g}\left[\int_{0}^{\eta(x)} \rho_{\mathrm{i}}(1-p)[\eta(x)-\delta] b(x, \delta) d \delta-\int_{0}^{s_{\mathrm{i}} \eta(x)} \rho(1-p)\left[s_{\mathrm{i}} \eta(x)-\delta\right] b(x, \delta) d \delta\right] .
\end{aligned}
$$

For a rectangular channel, $b$ is constant in the vertical and equal to the top width $B$. Therefore

$$
F_{\mathrm{pp}}{ }^{\prime}=K_{\mathrm{p}} g B(1-p)\left[\int_{0}^{\eta(x)} s_{\mathrm{i}} \rho[\eta(x)-\delta] d \delta-\int_{0}^{s_{\mathrm{i}} \eta(x)} \rho\left[s_{\mathrm{i}} \eta(x)-\delta\right] d \delta\right] .
$$

Integration yields

$$
F_{\mathrm{pp}}^{\prime}=K_{\mathrm{p}} g B(1-p) s_{\mathrm{i}} \rho\left(1-s_{\mathrm{i}}\right) \frac{\eta^{2}}{2} .
$$

The time integral of this net normal force in the $x$-direction is

$$
\int_{t_{1}}^{t_{2}} F_{\mathrm{pp}} d t=\int_{t_{1}}^{t_{2}}\left(F_{\mathrm{pp}}{ }^{\prime}-F_{\mathrm{pp}}{ }^{\prime \prime}\right) d t=g s_{\mathrm{i}}\left(1-s_{\mathrm{i}}\right) \int_{t_{1}}^{t_{2}}\left[\left(\rho I_{3}\right)_{\mathrm{x}_{1}}-\left(\rho I_{3}\right)_{\mathrm{x}_{2}}\right] d t
$$

where

$$
I_{3}=K_{\mathrm{p}} B \frac{\eta^{2}}{2}(1-p)
$$

It should be recognized that, by setting the value of $k_{1}=K_{p}$ in eq 85 , the jam is considered to be at its strength limit. A jam at rest could probably experience $k_{1}$ values ranging from $K_{\mathrm{a}}$, the active pressure coefficient, to $K_{\mathrm{p}}$, the passive pressure coefficient. For a cover in motion, $k_{1}$ most likely depends on ice velocity. More research is needed to determine the proper values of $k_{1}$ to be used in different states of ice motion.

An important further force is a shear stress $\tau_{x y}$, produced by ice grinding against the banks or channel sides. It relates directly to the normal stress in the $x$-direction at failure, i.e.

$$
\tau_{\mathrm{xy}}=\sigma_{\mathrm{x}} k_{0} \lambda=\overline{\sigma_{\mathrm{v}}} k_{0} \lambda K_{\mathrm{p}}
$$

where $k_{0}$ is the coefficient of lateral thrust (percentage of normal stress acting in the $x$-direction that would act in the $z$ - or cross-channel direction) and $\lambda$ is the sliding coefficient of ice on ice at the banks. Bank shear stress acts equally at either bank (for one-dimensional formulations, at least) and may vary between section $x_{1}$ and $x_{2}$. The force from the shear at the banks can be obtained from eq 84 and 92, i.e.

$$
F_{\text {is }}=2 \int_{x_{1}}^{x_{2}} k_{0} \lambda K_{\mathrm{p}} s_{\mathrm{i}} \rho g(1-p)\left(1-s_{\mathrm{i}}\right) \frac{\eta^{2}}{2} d x
$$

The time integral of this force is 


$$
\int_{t_{1}}^{t_{2}} F_{\mathrm{is}} d t=g s_{\mathrm{i}}\left(1-s_{\mathrm{i}}\right)(1-p) \int_{t_{1} x_{1}}^{t_{2} x_{2}} \rho I_{4} d x d t
$$

where

$$
I_{4}=k_{0} \lambda K_{\mathrm{p}} \eta^{2} .
$$

Therefore, the full conservation of momentum equation for the ice cover becomes

$$
\Delta M-M_{\mathrm{f}}=\int_{t_{1}}^{t_{2}} F_{\mathrm{p} 2} d t+\int_{t_{1}}^{t_{2}} F_{\mathrm{g} 3} d t+\int_{t_{1}}^{t_{2}} F_{\mathrm{fi}} d t+\int_{t_{1}}^{t_{2}} F_{\mathrm{pp}} d t-\int_{t_{1}}^{t_{2}} F_{\mathrm{si}} d t
$$

or

$$
\begin{aligned}
& \int_{x_{1}}^{x_{2}}\left[\left(s_{\mathrm{i}} \rho v A_{\mathrm{i}}\right)_{\mathrm{t}_{2}}-\left(s_{\mathrm{i}} \rho v A_{\mathrm{i}}\right)_{\mathrm{t}_{1}}\right] d x-\int_{t_{1}}^{t_{2}}\left[\left(\rho v^{2} A_{\mathrm{i}} s_{\mathrm{i}}\right)_{\mathrm{x}_{1}}-\left(\rho v^{2} A_{\mathrm{i}} s_{\mathrm{i}}\right)_{\mathrm{x}_{2}}\right] d t \\
& =g \int_{t_{1}}^{t_{2}}\left[\left(\rho I_{2}\right)_{\mathrm{x}_{1}}-\left(\rho I_{2}\right)_{\mathrm{x}_{2}}\right] d t+g \iint_{t_{1} x_{1}}^{t_{2} x_{2}} \rho A_{\mathrm{i}} s_{\mathrm{i}}\left(s_{\mathrm{o}}-\frac{\partial d}{\partial x}\right) d x d t \\
& +\int_{t_{1} x_{1}}^{t_{2} x_{2}} \rho \frac{f_{\mathrm{i}} P_{\mathrm{i}}}{8}(u-v)^{2} d x d t+g s_{\mathrm{i}}\left(1-s_{\mathrm{i}}\right) \int_{t_{1}}^{t_{2}}\left[\left(\rho I_{3}\right)_{\mathrm{x}_{1}}-\left(\rho I_{3}\right)_{\mathrm{x}_{2}}\right] d t \\
& -g s_{\mathrm{i}}\left(1-s_{\mathrm{i}}\right)(1-p) \iint_{t_{1} x_{1}}^{t_{1} x_{2}} \rho I_{4} d x d t .
\end{aligned}
$$

With $\rho$ being a constant

$$
\begin{aligned}
& \int_{x_{1}}^{x_{2}}\left[\left(s_{\mathrm{i}} v A_{\mathrm{i}}\right)_{\mathrm{t}_{2}}-\left(s_{\mathrm{i}} \mathrm{v} A_{\mathrm{i}}\right)_{\mathrm{t}_{1}}\right] d x-\int_{t_{1}}^{t_{2}}\left[\left(v^{2} A_{\mathrm{i}} s_{\mathrm{i}}\right)_{\mathrm{x}_{1}}-\left(v^{2} A_{\mathrm{i}} \mathrm{s}_{\mathrm{i}}\right)_{\mathrm{x}_{2}}\right] d t \\
& =g \int_{t_{1}}^{t_{2}}\left[\left(I_{2}\right)_{\mathrm{x}_{1}}-\left(I_{2}\right)_{\mathrm{x}_{2}}\right] d t+g \iint_{t_{1} x_{1}}^{t_{2} x_{2}} A_{\mathrm{i}} s_{\mathrm{i}}\left(s_{\mathrm{o}}-\frac{\partial d}{\partial x}\right) d x d t+\iint_{t_{1} x_{1}}^{t_{2} x_{2}} \frac{f_{\mathrm{i}} P_{\mathrm{i}}}{8}(u-v)^{2} d x d t \\
& +g s_{\mathrm{i}}\left(1-s_{\mathrm{i}}\right) \int_{t_{1}}^{t_{2}}\left[\left(I_{3}\right)_{\mathrm{x}_{1}}-\left(I_{3}\right)_{\mathrm{x}_{2}}\right] d t-g s_{\mathrm{i}}\left(1-s_{\mathrm{i}}\right)(1-p) \int_{t_{1} \int_{1} \int_{1} I_{4} d x d t .}^{t_{1} x_{2}}
\end{aligned}
$$

The integral relations given by eq 35, 37, 66, and 98 are valid for a channel of constant rectangular cross section. Substituting rectangular channel cross-section relationships for $S_{\mathrm{f}}, I_{1}, I_{2}, I_{3}$, and $I_{4}$ (i.e., $A=B d$ and $A_{\mathrm{i}}=B \eta$ ), then canceling out common terms, produces the conservation of mass and momentum equations in their integral form.

Conservation of water mass is

$$
\int_{t_{1}}^{t_{2}}\left[(u d)_{\mathrm{x}_{2}}-(u d)_{\mathrm{x}_{1}}\right] d t+\int_{x_{1}}^{x_{2}}\left[(d)_{\mathrm{t}_{2}}-(d)_{\mathrm{t}_{1}}\right] d x=0 .
$$

Conservation of ice mass is

$$
\int_{t_{1}}^{t_{2}}\left[(v \eta)_{x_{2}}-(v \eta)_{x_{1}}\right] d t+\int_{x_{1}}^{x_{2}}\left[(\eta)_{t_{2}}-(\eta)_{t_{1}}\right] d x=0 .
$$

Conservation of water momentum is 


$$
\begin{aligned}
& \int_{x_{1}}^{x_{2}}\left[(u d)_{\mathrm{t}_{2}}-(u d)_{\mathrm{t}_{1}}\right] d x+\int_{t_{1}}^{t_{2}}\left[\left(u^{2} d\right)_{\mathrm{x}_{2}}-\left(u^{2} d\right)_{\mathrm{x}_{1}}\right] d t \\
& +g \int_{t_{1}}^{t_{2}}\left[\left(\frac{d^{2}}{2}+d s_{\mathrm{i}} \eta\right)_{\mathrm{x}_{2}}-\left(\frac{d^{2}}{2}+d s_{\mathrm{i}} \eta\right)_{\mathrm{x}_{1}}\right] d t-g \iint_{t_{1} x_{1}}^{t_{2} x_{2}} \eta s_{\mathrm{i}} \frac{\partial d}{\partial x} d x d t \\
& -g \int_{t_{1} x_{1}}^{t_{2} x_{2}} d S_{\mathrm{o}} d x d t+\int_{t_{1} x_{1}}^{t_{2} x_{2}} \frac{f_{\mathrm{b}} u^{2}(B+2 d)}{8 B}\left[1+\frac{f_{\mathrm{i}}}{f_{\mathrm{b}}} \frac{B}{(B+2 d)}\left(\frac{u-v}{u}\right)^{2}\right] d x d t=0 .
\end{aligned}
$$

Conservation of ice momentum is

$$
\begin{aligned}
& \int_{x_{1}}^{x_{2}}\left[(v \eta)_{\mathrm{t}_{2}}-(v \eta)_{\mathrm{t}_{1}}\right] d x+\int_{t_{1}}^{t_{2}}\left[\left(v^{2} \eta\right)_{\mathrm{x}_{2}}-\left(v^{2} \eta\right)_{\mathrm{x}_{1}}\right] d t+g s_{\mathrm{i}} \int_{t_{1}}^{t_{2}}\left[\left(\frac{\eta^{2}}{2}\right)_{\mathrm{x}_{2}}-\left(\frac{\eta^{2}}{2}\right)_{\mathrm{x}_{1}}\right] d t \\
& -g \int_{t_{1} x_{1}}^{t_{2} x_{2}} \eta\left(S_{\mathrm{O}}-\frac{\partial d}{\partial x}\right) d x d t+g\left(1-s_{\mathrm{i}}\right) \int_{t_{1}}^{t_{2}}\left[\left(\frac{K_{\mathrm{p}}(1-p) \eta^{2}}{2}\right)_{\mathrm{x}_{2}}-\left(\frac{K_{\mathrm{p}}(1-p) \eta^{2}}{2}\right)\right] d t \\
& -\frac{1}{8 s_{\mathrm{i}}} \int_{t_{1} x_{1}}^{t_{2} x_{2}} f_{\mathrm{i}}(u-v)^{2} d x d t+\frac{g\left(1-s_{\mathrm{i}}\right)}{B} \int_{t_{1} x_{1}}^{t_{2} x_{2}} k_{0} \lambda K_{\mathrm{p}}(1-p) \eta^{2} d x d t=0 .
\end{aligned}
$$

\section{Discretization of the system of equations}

The equations are transformed from their integral form into a differential form to facilitate the discretization needed to proceed with numerically simulating jams. By assuming the dependent variables to be continuous, differentiable functions enables their expansion as Taylor series. The terms in the conservation of water mass equation become thereby

$$
\begin{aligned}
& (d)_{\mathrm{t}_{2}}=(d)_{\mathrm{t}_{1}}+\frac{\partial(d)}{\partial t} \Delta t+\frac{\partial^{2}(d)}{\partial t^{2}} \frac{\Delta t^{2}}{2}+\ldots \\
& (u d)_{\mathrm{x}_{2}}=(u d)_{\mathrm{x}_{1}}+\frac{\partial(u d)}{\partial x} \Delta x+\frac{\partial^{2}(u d)}{\partial x^{2}} \frac{\Delta x^{2}}{2}+\ldots
\end{aligned}
$$

Then, by retaining only the first-order terms and assuming that the increments $\Delta x$ and $\Delta t$ approach zero

$$
\begin{array}{ll}
t_{2} \stackrel{\lim }{\longrightarrow} t_{1} & \int_{x_{1}}^{x_{2}}\left[(d)_{t_{2}}-(d)_{t_{1}}\right] d x=\iint_{x_{1}}^{x_{2}} \int \frac{t_{1}}{\partial t} \frac{\partial(d)}{\partial t} d t d x \\
x_{2} \stackrel{\lim }{\longrightarrow} x_{1} & \int_{t_{1}}^{t_{2}}\left[(u d)_{\mathrm{x}_{2}}-(u d)_{\mathrm{x}_{1}}\right] d t=\int_{t_{1} x_{1}}^{t_{2} x_{2}} \frac{\partial(u d)}{\partial x} d x d t .
\end{array}
$$

Consequently, the conservation of water mass equation reduces to

$$
\int_{t_{1} x_{1}}^{t_{2} x_{2}}\left[\frac{\partial(d)}{\partial t}+\frac{\partial(u d)}{\partial x}\right] d x d t=0 .
$$

If this equation is to hold everywhere in the $(x, t)$ plane, then it will hold for an infinitely small volume, such that the eq 105 can be rewritten as 


$$
\frac{\partial(d)}{\partial t}+\frac{\partial(u d)}{\partial x}=0
$$

In a similar fashion, the conservation of ice mass equation becomes

$$
\frac{\partial(\eta)}{\partial t}+\frac{\partial(v \eta)}{\partial x}=0
$$

The conservation of water momentum and conservation of ice momentum equations contain a combination of single and double integrals. By Taylor series expansion as above, the conservation of water momentum becomes

$$
\begin{aligned}
& \int_{t_{1}}^{t_{2} x_{1}}\left[\frac{\partial(u d)}{\partial t}+\frac{\partial\left(u^{2} d\right)}{\partial x}+g \frac{\partial\left(\frac{d^{2}}{2}+d s_{\mathrm{i}} \eta\right)}{\partial x}\right] d x d t \\
& -g \int_{t_{1} x_{1}}^{t_{2} x_{2}}\left[\eta s_{\mathrm{i}} \frac{\partial d}{\partial x}+d S_{\mathrm{o}}-\frac{f_{\mathrm{b}} u^{2}(B+2 d)}{8 g B}\left(1+\frac{f_{\mathrm{i}}}{f_{\mathrm{b}}} \frac{B}{(B+2 d)}\left[\frac{u-v}{u}\right]^{2}\right)\right] d x d t=0
\end{aligned}
$$

and the conservation of ice momentum becomes

$$
\begin{aligned}
& \int_{t_{1} x_{1}}^{t_{2} x_{2}}\left[\frac{\partial(v \eta)}{\partial t}+\frac{\partial\left(v^{2} \eta\right)}{\partial x}+g s_{\mathrm{i}} \frac{\partial}{\partial x}\left(\frac{\eta^{2}}{2}\right)+g\left(1-s_{\mathrm{i}}\right) \frac{\partial}{\partial x}\left(K_{\mathrm{p}}(1-p)\left[\frac{\eta^{2}}{2}\right]\right)\right] d x d t \\
& +g \int_{t_{1} x_{1}}^{t_{2} x_{2}}\left[\frac{\left(1-s_{\mathrm{i}}\right)}{B} k_{0} \lambda K_{\mathrm{p}}(1-p) \eta^{2}-\eta S_{\mathrm{o}}+\eta \frac{\partial d}{\partial x}-\frac{f_{i}}{8 g s_{\mathrm{i}}}(u-v)^{2}\right] d x d t=0 .
\end{aligned}
$$

Under the assumption that these equations also hold everywhere in the $(x, t)$ plane, the conservation of water momentum equation is

$$
\begin{aligned}
& \frac{\partial(u d)}{\partial t}+\frac{\partial\left(u^{2} d\right)}{\partial x}+g \frac{\partial}{\partial x}\left(\frac{d^{2}}{2}+d s_{\mathrm{i}} \eta\right)-g s_{\mathrm{i}} \eta \frac{\partial d}{\partial x}-g d S_{\mathrm{o}} \\
& +\frac{f_{\mathrm{b}} u^{2}(B+2 d)}{8 B}\left[1+\frac{f_{\mathrm{i}}}{f_{\mathrm{b}}} \frac{B}{(B+2 d)}\left(\frac{u-v}{u}\right)^{2}\right]=0 .
\end{aligned}
$$

In a similar manner, the conservation of ice momentum equation becomes

$$
\begin{aligned}
& \frac{\partial(v \eta)}{\partial t}+\frac{\partial\left(v^{2} \eta\right)}{\partial x}+g s_{\mathrm{i}} \frac{\partial}{\partial x}\left(\frac{\eta^{2}}{2}\right)+g\left(1-s_{\mathrm{i}}\right) \frac{\partial}{\partial x}\left(K_{\mathrm{p}}(1-p) \frac{\eta^{2}}{2}\right) \\
& +\frac{g\left(1-s_{\mathrm{i}}\right)}{B} k_{0} \lambda K_{\mathrm{p}}(1-p) \eta^{2}-g \eta S_{\mathrm{o}}+g \eta \frac{\partial d}{\partial x}-\frac{f_{\mathrm{i}}}{8 s_{\mathrm{i}}}(u-v)^{2}=0 .
\end{aligned}
$$

The partial derivatives with combined dependent variables in the foregoing equations are then expanded to separate the variables. The conservation of water mass equation becomes

$$
\frac{\partial d}{\partial t}+u \frac{\partial d}{\partial x}+d \frac{\partial u}{\partial x}=0 .
$$


The conservation of ice mass equation becomes

$$
\frac{\partial \eta}{\partial t}+v \frac{\partial \eta}{\partial x}+\eta \frac{\partial v}{\partial x}=0
$$

Expanding the terms in eq 110, combining with eq 112, and dividing by $d$, the conservation of water momentum equation becomes

$$
\begin{aligned}
& \frac{\partial u}{\partial t}+u \frac{\partial u}{\partial x}+g \frac{\partial d}{\partial x}+g S_{\mathrm{i}} \frac{\partial \eta}{\partial x}-g S_{\mathrm{o}} \\
& +\frac{f_{b} u^{2}(B+2 d)}{8 B d}\left[1+\frac{f_{\mathrm{i}}}{f_{\mathrm{b}}} \frac{B}{(B+2 d)}\left(\frac{u-v}{u}\right)^{2}\right]=0 .
\end{aligned}
$$

Similarly, after expansion of the partials in eq 111, combining with eq 113, and dividing by $\eta$, the conservation of ice momentum equation becomes

$$
\begin{aligned}
& \frac{\partial v}{\partial t}+v \frac{\partial v}{\partial x}+g s_{\mathrm{i}} \frac{\partial \eta}{\partial x}+g \frac{\partial d}{\partial x}+g\left(1-s_{\mathrm{i}}\right) K_{\mathrm{p}}(1-p) \frac{\partial \eta}{\partial x} \\
& +\frac{g\left(1-s_{\mathrm{i}}\right)}{B} k_{0} \lambda K_{\mathrm{p}}(1-p) \eta-g S_{\mathrm{o}}-\frac{f_{\mathrm{i}}}{8 s_{\mathrm{i}} \eta}(u-v)^{2}=0 .
\end{aligned}
$$

Figure 30 shows the computational grid used for the numerical simulations in this study. The discretization of the governing equations assumes that the values of the dependent variables and functions of the variables between the computational grid points can be expressed in terms of the values at the grid points. A function $f(x, t)$ within the space interval $j$ and $(j+1)$ is replaced by the following weighted average

$$
f\left(x, t_{\mathrm{n}}\right)=\Psi f_{\mathrm{j}+1}^{n}+(1-\Psi) f_{\mathrm{j}}^{n}
$$

and

$$
f\left(x, t_{\mathrm{n}+1}\right)=\Psi f_{\mathrm{j}+1}^{n+1}+(1-\Psi) f_{\mathrm{j}}^{n+1}
$$

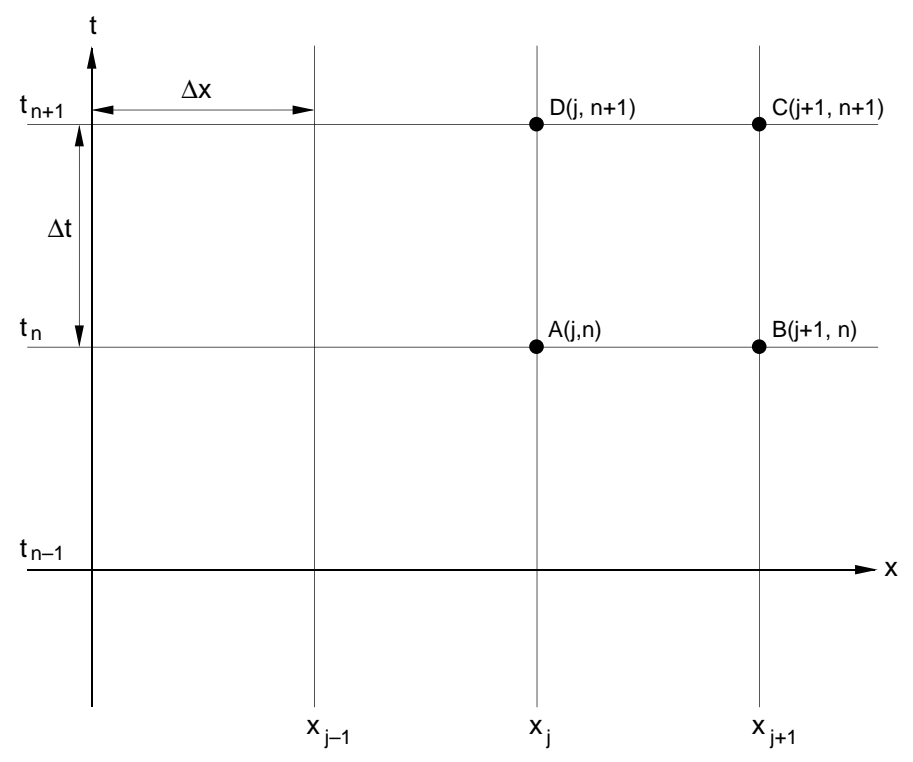

Figure 30. Computational grid used for the numerical simulations. 
where $x_{\mathrm{j}} \leq x \leq x_{\mathrm{j}+1}$. Similarly, for the time interval $t_{\mathrm{n}} \leq t \leq t_{\mathrm{n}+1}$

$$
f\left(x_{\mathrm{j}}, t\right)=\theta f_{\mathrm{j}}^{n+1}+(1-\theta) f_{\mathrm{j}}^{n}
$$

and

$$
f\left(x_{j+1}, t\right)=\theta f_{j+1}^{n+1}+(1-\theta) f_{j+1}^{n}
$$

where $0 \leq \theta \leq 1$ and $0 \leq \Psi \leq 1$ are weighting factors. Subscripts denote the $x$ location while superscripts denote time level. By use of these relations, the finite difference equations that approximate the conservation laws expressed by the differential eq 112, 113, 114, and 115 can be developed. When $\Psi=0.5$, the resulting equations represent the Preissmann four-point scheme of finite differences. In the discretization, the derivatives of a function $f(x, t)$ are estimated as

$$
\frac{\partial f}{\partial t}=\frac{\left(f_{\mathrm{j}+1}^{n+1}+f_{\mathrm{j}}^{n+1}\right)-\left(f_{\mathrm{j}+1}^{n}+f_{\mathrm{j}}^{n}\right)}{2 \Delta t}
$$

and

$$
\frac{\partial f}{\partial x}=\frac{\theta\left(f_{\mathrm{j}+1}^{n+1}-f_{\mathrm{j}}^{n+1}\right)+(1-\theta)\left(f_{\mathrm{j}+1}^{n}-f_{\mathrm{j}}^{n}\right)}{\Delta x}
$$

and the values of the variables, or the functions themselves, are estimated as

$$
f(x, t)=\frac{\theta\left(f_{\mathrm{j}}^{n+1}+f_{\mathrm{j}+1}^{n+1}\right)+(1-\theta)\left(f_{\mathrm{j}}^{n}+f_{\mathrm{j}+1}^{n}\right)}{2} .
$$

The above rules of discretization can be used to recast the conservation of water mass equation as

$$
\begin{aligned}
& \frac{\left(d_{j+1}^{n+1}+d_{j}^{n+1}\right)-\left(d_{j+1}^{n}+d_{j}^{n}\right)}{2 \Delta t}+\bar{u}\left[\frac{\theta\left(d_{j+1}^{n+1}-d_{j}^{n+1}\right)+(1-\theta)\left(d_{j+1}^{n}-d_{j}^{n}\right)}{\Delta x}\right] \\
& +\bar{d}\left[\frac{\theta\left(u_{j+1}^{n+1}-u_{j}^{n+1}\right)+(1-\theta)\left(u_{j+1}^{n}-u_{j}^{n}\right)}{\Delta x}\right]=0
\end{aligned}
$$

where

$$
\bar{u}=\frac{\theta\left(u_{j+1}^{n+1}+u_{j}^{n+1}\right)+(1-\theta)\left(u_{j+1}^{n}+u_{j}^{n}\right)}{2}
$$

and

$$
\bar{d}=\frac{\theta\left(d_{j+1}^{n+1}+d_{j}^{n+1}\right)+(1-\theta)\left(d_{j+1}^{n}+d_{j}^{n}\right)}{2} .
$$

Similarly, the conservation of ice mass equation becomes

$$
\begin{aligned}
& \frac{\left(\eta_{j+1}^{n+1}+\eta_{j}^{n+1}\right)-\left(\eta_{j+1}^{n}+\eta_{j}^{n}\right)}{2 \Delta t}+v\left[\frac{\theta_{i}\left(\eta_{j+1}^{n+1}-\eta_{j}^{n+1}\right)+\left(1-\theta_{i}\right)\left(\eta_{j+1}^{n}-\eta_{j}^{n}\right)}{\Delta x}\right] \\
& +\bar{\eta}\left[\frac{\theta_{i}\left(v_{j+1}^{n+1}-v_{j}^{n+1}\right)+\left(1-\theta_{i}\right)\left(v_{j+1}^{n}-v_{j}^{n}\right)}{\Delta x}\right]=0
\end{aligned}
$$


where $\theta_{\mathrm{i}}$ is a weighting factor for the ice variables

$$
\bar{v}=\frac{\theta_{i}\left(v_{j+1}^{n+1}+v_{j}^{n+1}\right)+\left(1-\theta_{i}\right)\left(v_{j+1}^{n}+v_{j}^{n}\right)}{2}
$$

and

$$
\bar{\eta}=\frac{\theta_{i}\left(\eta_{j+1}^{n+1}+\eta_{j}^{n+1}\right)+\left(1-\theta_{i}\right)\left(\eta_{j+1}^{n}+\eta_{j}^{n}\right)}{2} .
$$

The conservation of water momentum equation transforms to

$$
\begin{aligned}
& \frac{\left(u_{\mathrm{j}+1}^{n+1}+u_{\mathrm{j}}^{n+1}\right)-\left(u_{\mathrm{j}+1}^{n}+u_{\mathrm{j}}^{n}\right)}{2 \Delta t}+\bar{u}\left[\frac{\theta\left(u_{\mathrm{j}+1}^{n+1}-u_{\mathrm{j}}^{n+1}\right)+(1-\theta)\left(u_{\mathrm{j}+1}^{n}-u_{\mathrm{j}}^{n}\right)}{\Delta x}\right] \\
& +\frac{f_{\mathrm{b}} \overline{u^{2}}}{8 \bar{d}}+\frac{f_{\mathrm{b}} \overline{u^{2}}}{4 B}+\frac{f_{\mathrm{i}} \overline{(u-v)^{2}}}{8 \bar{d}}+g\left[\frac{\theta\left(d_{\mathrm{j}+1}^{n+1}-d_{\mathrm{j}}^{n+1}\right)+(1-\theta)\left(d_{\mathrm{j}+1}^{n}-d_{\mathrm{j}}^{n}\right)}{\Delta x}\right] \\
& +g S_{\mathrm{i}}\left[\frac{\theta\left(\eta_{\mathrm{j}+1}^{n+1}-\eta_{\mathrm{j}}^{n+1}\right)+(1-\theta)\left(\eta_{\mathrm{j}+1}^{n}-\eta_{\mathrm{j}}^{n}\right)}{\Delta x}\right]-g S_{\mathrm{o}}=0
\end{aligned}
$$

where $\bar{u}, \bar{d}$, and $+\bar{v}$ are defined as above. Note that the last term in eq 114 has been expanded for clarity and that the discretization of these terms containing squared variables are weighted averages of the squared terms. They are not squares of the weighted averages of the variables. This minor detail preserves the physical meaning of the terms. Those terms are

$$
\overline{u^{2}}=\frac{\theta\left[\left(u^{2}\right)_{j+1}^{n+1}+\left(u^{2}\right)_{j}^{n+1}\right]+(1-\theta)\left[\left(u^{2}\right)_{j+1}^{n}+\left(u^{2}\right)_{j}^{n}\right]}{2}
$$

and

$$
\overline{(u-v)^{2}}=\frac{\theta\left[\left([u-v]^{2}\right)_{j+1}^{n+1}+\left([u-v]^{2}\right)_{j}^{n+1}\right]+(1-\theta)\left[\left([u-v]^{2}\right)_{j+1}^{n}+\left([u-v]^{2}\right)_{j}^{n}\right]}{2} .
$$

The conservation of ice momentum equation transforms to

$$
\begin{aligned}
& \frac{\left(v_{j+1}^{n+1}+v_{j}^{n+1}\right)-\left(v_{j+1}^{n}+v_{j}^{n}\right)}{2 \Delta t}+\bar{v}\left[\frac{\theta_{i}\left(v_{j+1}^{n+1}-v_{j}^{n+1}\right)+\left(1-\theta_{i}\right)\left(v_{j+1}^{n}-v_{j}^{n}\right)}{\Delta x}\right] \\
& +\left(g\left(1-s_{i}\right) K_{p}(1-p)+g s_{i}\right)\left[\frac{\theta_{i}\left(\eta_{j+1}^{n+1}-\eta_{j}^{n+1}\right)+\left(1-\theta_{i}\right)\left(\eta_{j+1}^{n}-\eta_{j}^{n}\right)}{\Delta x}\right] \\
& +g\left[\frac{\theta\left(d_{j+1}^{n+1}-d_{j}^{n+1}\right)+(1-\theta)\left(d_{j+1}^{n}-d_{j}^{n}\right)}{\Delta x}\right]-g S_{o} \\
& +\frac{g\left(1-s_{i}\right) k_{0} \lambda K_{p}(1-p) \bar{\eta}}{B}-\frac{f_{i}}{8 s_{i} \bar{\eta}} \overline{(u-v)^{2}}=0 .
\end{aligned}
$$




\section{Solution of the system of equations}

A Newton-Raphson iteration procedure was used to solve the system of equations given above. If an equation is designated as

$$
F=F\left(d_{j}, \eta_{j}, u_{j}, v_{j}, d_{j+1}, \eta_{j+1}, u_{j+1}, v_{j+1}\right)
$$

then for any two points ' $j$ ' and ' $j+1$ ' at any time, it can be expanded as a Taylor Series

$$
\begin{aligned}
& { }^{m+1} F={ }^{m} F+\left.\frac{\partial F}{\partial d_{\mathrm{j}}}\right|_{m} \Delta d_{\mathrm{j}}+\left.\frac{\partial F}{\partial d_{\mathrm{j}+1}}\right|_{m} \Delta d_{\mathrm{j}+1}+\left.\frac{\partial F}{\partial \eta_{\mathrm{j}}}\right|_{m} \Delta \eta_{\mathrm{j}}+\left.\frac{\partial F}{\partial \eta_{\mathrm{j}+1}}\right|_{m} \Delta \eta_{\mathrm{j}+1}+ \\
& \left.\frac{\partial F}{\partial u_{\mathrm{j}}}\right|_{m} \Delta u_{\mathrm{j}}+\left.\frac{\partial F}{\partial u_{\mathrm{j}+1}}\right|_{m} \Delta u_{\mathrm{j}+1}+\left.\frac{\partial F}{\partial v_{\mathrm{j}}}\right|_{m} \Delta v_{\mathrm{j}}+\left.\frac{\partial F}{\partial v_{\mathrm{j}+1}}\right|_{m} \Delta v_{\mathrm{j}+1}
\end{aligned}
$$

where ' $m$ ' and ' $m+1$ ' indicate the iteration level and ' $j$ ' and ' $j+1$ ' indicate the $x$ location. The partial derivatives are evaluated on the basis of the values of the variables after the ' $m$ th' iteration. Therefore, each equation can be transformed into a linear equation in terms of the eight unknowns: $\Delta d_{j}, \Delta \eta_{j}, \Delta u_{j}, \Delta v_{j}, \Delta d_{j+1}, \Delta \eta_{j+1}, \Delta u_{j+1}$, and $\Delta v_{j+1}$. The goal of the method is to solve for the change between ' $m$ ' and ' $m+1$ ' such that ${ }^{m+1} F \Rightarrow 0$. It can also be seen that ${ }^{m} F$ is a function of the final values of the variables at time ' $n$ ' and the values for the ' $m$ th' iteration at time ' $n+1$, ' which are all known values. The transformed equations are then put into the form

$$
\begin{aligned}
& K={ }^{m+1} F-{ }^{m} F \\
& =a \Delta d_{j}+b \Delta \eta_{j}+c \Delta u_{j}+d \Delta v_{j}+e \Delta d_{j+1}+f \Delta \eta_{j+1}+g \Delta u_{j+1}+h \Delta v_{j+1}
\end{aligned}
$$

where

$$
\begin{array}{llll}
a=\frac{\partial F}{\partial d_{\mathrm{j}}}, & b=\frac{\partial F}{\partial \eta_{\mathrm{j}}}, & c=\frac{\partial F}{\partial u_{\mathrm{j}}}, & d=\frac{\partial F}{\partial v_{\mathrm{j}}}, \\
e=\frac{\partial F}{\partial d_{\mathrm{j}+1}}, & f=\frac{\partial F}{\partial \eta_{\mathrm{j}+1}}, & g=\frac{\partial F}{\partial u_{\mathrm{j}+1}}, & h=\frac{\partial F}{\partial v_{\mathrm{j}+1}} .
\end{array}
$$

Since $K={ }^{m+1} F-{ }^{m} F$, and the goal is to achieve ${ }^{m+1} F \Rightarrow 0$

$$
K=-{ }^{m} F
$$

For example, the conservation of water mass equation becomes

$$
\begin{aligned}
& F=\frac{\left(d_{\mathrm{j}+1}^{n+1}+d_{\mathrm{j}}^{n+1}\right)-\left(d_{\mathrm{j}+1}^{n}+d_{\mathrm{j}}^{n}\right)}{2 \Delta t}+\bar{u}\left[\frac{\theta\left(d_{\mathrm{j}+1}^{n+1}-d_{\mathrm{j}}^{n+1}\right)+(1-\theta)\left(d_{\mathrm{j}+1}^{n}-d_{\mathrm{j}}^{n}\right)}{\Delta x}\right]+ \\
& \bar{d}\left[\frac{\theta\left(u_{\mathrm{j}+1}^{n+1}-u_{\mathrm{j}}^{n+1}\right)+(1-\theta)\left(u_{\mathrm{j}+1}^{n}-u_{\mathrm{j}}^{n}\right)}{\Delta x}\right]=0
\end{aligned}
$$

and the derivatives are evaluated as 


$$
\begin{aligned}
& a=\frac{\partial F}{\partial d_{j}}=\frac{1}{2 \Delta t}-\frac{\bar{u} \theta}{\Delta x}+\frac{\theta}{2}\left[\frac{\theta\left(u_{j+1}^{n+1}-u_{j}^{n+1}\right)+(1-\theta)\left(u_{j+1}^{n}-u_{j}^{n}\right)}{\Delta x}\right] \\
& b=\frac{\partial F}{\partial \eta_{j}}=0 \\
& c=\frac{\partial F}{\partial u_{j}}=\frac{\theta}{2}\left[\frac{\theta\left(d_{j+1}^{n+1}-d_{j}^{n+1}\right)+(1-\theta)\left(d_{j+1}^{n}-d_{j}^{n}\right)}{\Delta x}\right]-\frac{\bar{d} \theta}{\Delta x} \\
& d=\frac{\partial F}{\partial v_{j}}=0 \\
& e=\frac{\partial F}{\partial d_{j+1}}=\frac{1}{2 \Delta t}+\frac{\bar{u} \theta}{\Delta x}+\frac{\theta}{2}\left[\frac{\theta\left(u_{j+1}^{n+1}-u_{j}^{n+1}\right)+(1-\theta)\left(u_{j+1}^{n}-u_{j}^{n}\right)}{\Delta x}\right] \\
& f=\frac{\partial F}{\partial \eta_{j+1}}=0 \\
& g=\frac{\partial F}{\partial u_{j+1}}=\frac{\theta}{2}\left[\frac{\theta\left(d_{j+1}^{n+1}-d_{j}^{n+1}\right)+(1-\theta)\left(d_{j+1}^{n}-d_{j}^{n}\right)}{\Delta x}\right]+\frac{\bar{d} \theta}{\Delta x} \\
& h=\frac{\partial F}{\partial v_{j}}=0 .
\end{aligned}
$$

The same procedure was followed for the other three equations, using the notations $F^{\prime}, F^{\prime \prime}, F^{\prime \prime \prime}, a^{\prime}, a^{\prime \prime}, a^{\prime \prime \prime}, \ldots, K^{\prime}, K^{\prime \prime}, K^{\prime \prime \prime}$ to distinguish among equations. The discretized equations and their derivatives are listed in Appendix A.

For each point, there are now four unknowns $(\Delta d, \Delta \eta, \Delta u$, and $\Delta v)$ or a total of $4 N$ unknowns, where $N$ is the number of cross sections. For each reach there are four linear equations or $4(N-1)$ equations. Four boundary condition equations are needed to close the system. The linear algebraic system can be represented as

$$
[A]\{\Delta Z\}=\{K\} \text { or }\{\Delta Z\}=[A]^{-1}\{K\}
$$

where

$$
\{\Delta Z\}=\left\{\Delta d_{1}, \Delta \eta_{1}, \Delta u_{1}, \Delta v_{1}, \Delta d_{2}, \Delta \eta_{2}, \ldots, \Delta d_{N}, \Delta \eta_{N}, \Delta u_{N}, \Delta v_{N}\right\}^{T}
$$

and

$$
\{K\}=\left\{K_{1}, K_{1}^{\prime}, K_{1}^{\prime \prime}, K_{1}^{\prime \prime \prime}, K_{2}, K_{2}^{\prime}, K_{2}^{\prime \prime}, K_{2}^{\prime \prime \prime}, \ldots, K_{\mathrm{N}}, K_{\mathrm{N}}^{\prime}, K_{\mathrm{N}}^{\prime \prime}, K_{\mathrm{N}}^{\prime \prime \prime}\right\}^{\mathrm{T}} .
$$

The coefficient matrix $[A]$ is depicted in Figure 31. $[A]$ is a banded coefficient matrix of 11 diagonals with the $x^{\prime}$ s signifying boundary conditions and the empty spaces filled with zeros. The system is solved using a decomposition-backsubstitution scheme to obtain the $\Delta Z$ terms. Subsequently, for the next iteration 


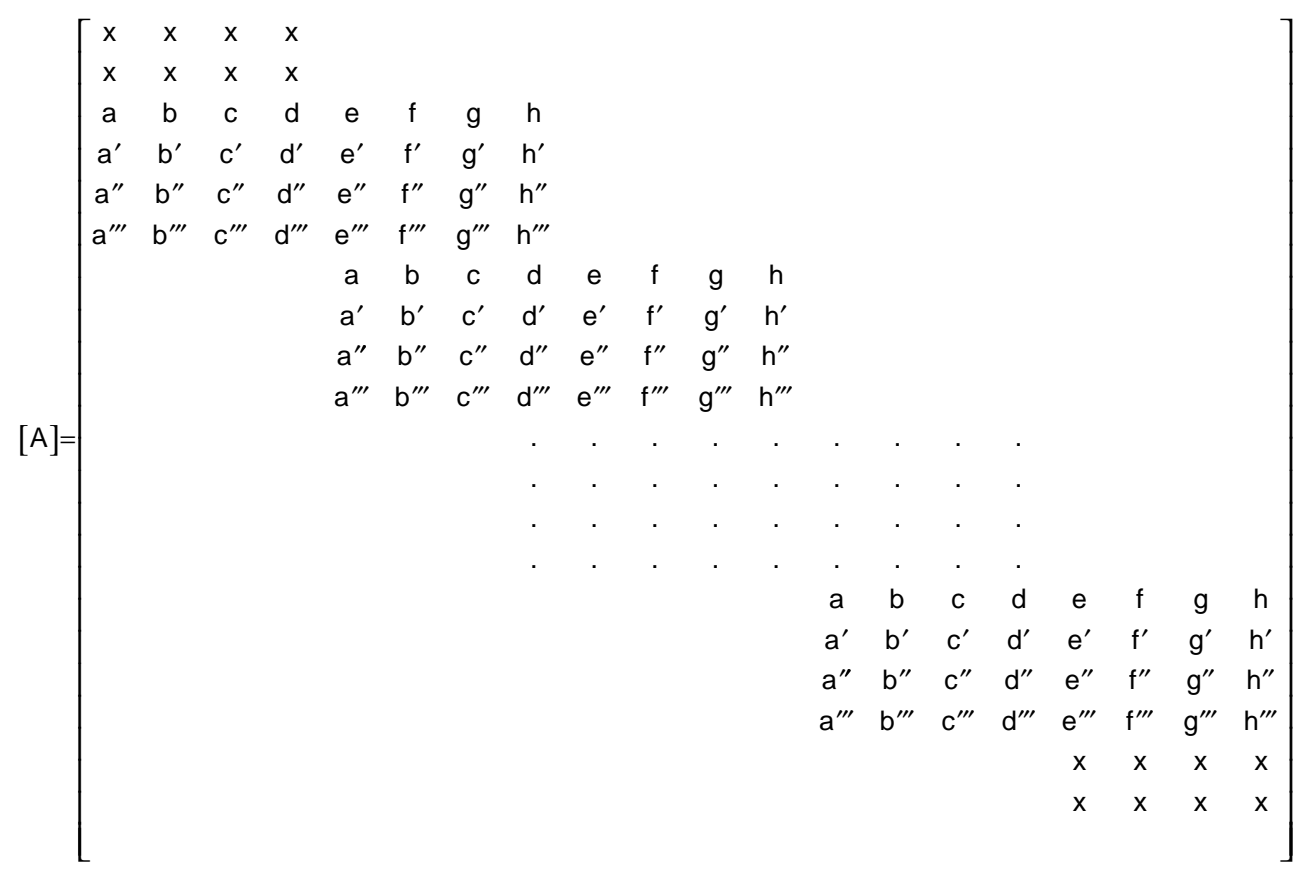

Figure 31. Coefficient matrix for the Newton-Raphson iteration procedure.

$$
{ }^{m+1} d_{\mathrm{j}}^{n+1}={ }^{m} d_{\mathrm{j}}^{n+1}+\Delta d_{\mathrm{j}}
$$

The values of the variables are updated in this way for the ' $m+1$ ' iteration and solved again until a specified tolerance is met. The tolerance is expressed in a least squares form

$$
T o l=\sum_{j=1}^{N-1}\left({ }^{m+1} F-{ }^{m} F\right)^{2}=\sum_{j=1}^{N}\left(\Delta^{\prime} s\right)^{2} .
$$

When the tolerance is met, the final ' $m+1$ ' iteration values are used as the values of the dependent variables for time ' $n+1$ '. The time is then incremented and the iteration procedure begins again. For the first iteration at a time step (' $m=0$ '), the initial values of the variables are taken to be equal to the final value of the variables at the previous time step.

\section{Ice cover stability, solution methods, and boundary conditions}

The conservation of ice momentum, as expressed in eq 102 or 115, is actually an equation expressing a unique balance of ice momentum and external forces acting on an ice jam element. Through assumption of the passive-pressure failure criteria and adopting the Rankine equation for $K_{\mathrm{p}}$ (the passive pressure coefficient), the equation is valid for a static element of an ice jam at its limit of stability. The equation is also valid for an ice jam element failing and in motion. In the former case, if the forces on a static element exceed the passive pressure limit, application of the equation (in concert with the other conservation equations) results in downstream ice movement. For an ice element in motion, application of the equation for a change in the forces may result in changes in ice velocity or thickness. For many cases, the 
net force applied to the ice cover element may be less than that "allowed" by the passive pressure limit. Application of the equation in this case would result in either ice motion upstream (negative ice momentum) or thinning of the jam to achieve a balance. In natural systems, thinning or upstream movement of ice rarely occurs. The forces exerted on a thickened, but static, section of jam are reduced. The jam simply remains in place with no change in thickness. In such a case when the forces exerted lead to a stable jam (i.e., below the passive pressure limit), the conservation of ice momentum equation must be adjusted so that negative ice velocities or thinning do not take place.

The computational procedure commences at the upstream end of the system, determining the stability of each reach at the beginning of each time step. The forces acting on the jam and water in the reach are assessed and compared with the maximum resistive force at the downstream end of the reach. The assumption of passive pressure limits the net force at the downstream end of each reach to that given by eq 89. If the sum of the forces (normal force at the upstream end of the reach, ice momentum, hydrostatic pressure, gravity, shear on the underside of the cover, and friction at the banks) is less than the passive pressure limit, the reach is deemed stable. The stability check continues in the downstream direction with the normal force at the upstream end of the next reach set equal to the net force at the downstream end of the current reach. The stability check assesses the stability of each cross section by comparing the net force and passive pressure resistance.

Modifications to the conservation equations of ice mass and ice momentum applied to each reach are necessary, depending on the stability conditions at the ends of each reach. One of the upstream boundary conditions of the system provides a relationship for the upstream ice thickness of the first reach. Thus, the conservation of ice mass equation provides a relationship for the upstream ice velocity of a reach. The conservation of ice momentum provides a relationship for the downstream ice thickness for a reach. One of the downstream boundary conditions of the system describes a relationship for the downstream ice velocity at the last reach. If a cross section is deemed to be stable, ice velocity in it will be zero, and the conservation of ice mass equation reverts to

$$
v_{j}^{n+1}=0 .
$$

Several combinations of upstream and downstream stability are possible for a reach (Fig. 32). For the first case, both upstream and downstream ends are unstable and the full equations are used. In the next case, both ends are deemed stable and, thus, the ice velocity equals zero at both ends, and eq 148 is used. Because there is no ice movement, the ice thickness must remain constant. Consequently, the conservation of ice momentum equation reverts to

$$
\eta_{j+1}^{n+1}-\eta_{j+1}^{n}=0
$$

In the third case of Figure 32, the downstream end is stable and, thus, ice velocity is equal to zero. But the upstream end is unstable and the full ice-continuity equation is required. Ice moves into the reach, thickening at the downstream end, and requiring use of the full ice momentum equation. The last case shown occurs when the ice is stable at the upstream end, but unstable at the downstream end. Again, eq 148 is used for the ice continuity equation, but the full ice momentum equation is required for defining the downstream thickness. 

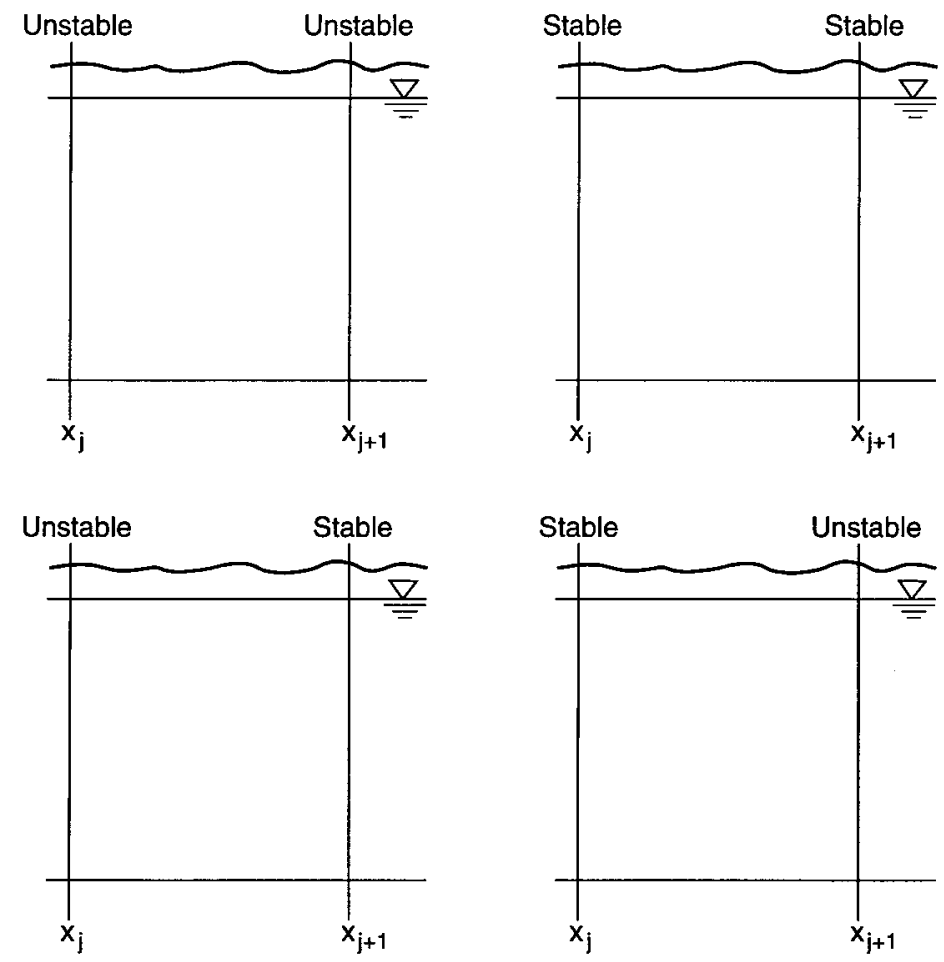

Figure 32. Conditions of ice jam stability for a computational reach.

Three modes of solution of the system of equations are presented below. The fully coupled mode comprises the simultaneous solution of all four dependent variables using the full equations and Newton-Raphson iteration technique presented above. The uncoupled or loosely coupled mode solves the water equations and ice equations separately and sequentially, as is done in the moving ice model of Tsai et al. (1988). The static-unsteady thickness mode first solves the water equations, then a modified version of the conservation of momentum equation not including the ice-momentum component. This latter procedure is comparable to the procedure used in stationary jam formulations.

\section{Fully coupled solution}

The fully coupled mode of solution uses the full equations of mass and momentum conservation for the water and ice with the simplifications mentioned above for sections and reaches that are determined to be stable. The four dependent variables are solved simultaneously using the Newton-Raphson iteration scheme mentioned above. The coefficient matrix, as depicted in Figure 31, is solved by decomposition-back-substitution using banded-matrix manipulation techniques. The coefficient matrix is a banded symmetrical matrix with five bands above and five below the main diagonal. Four equations for each reach relate the $4 \mathrm{~N}$ unknowns, where $N$ is the number of cross sections. Two boundary condition equations at the upstream end and two at the downstream end are required to close the system. The upstream boundary conditions are typically a water discharge relation (e.g., specified discharge) and an ice thickness relation (such as static equilibrium or specified thickness). The downstream boundary conditions are typically a specified depth relation (under ice) and an ice velocity relation. 


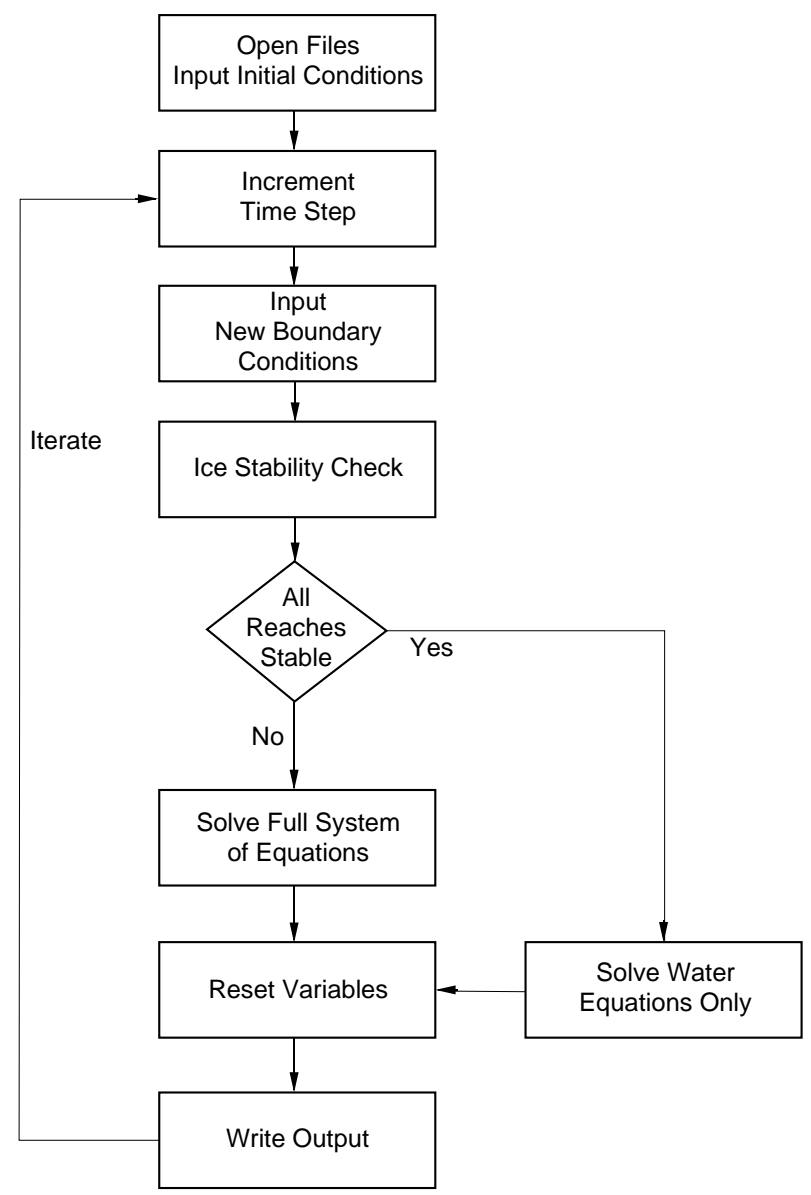

Figure 33. Block diagram for the fully coupled solution scheme.

The block diagram for the fully coupled solution scheme is presented in Figure 33. The initial conditions are read and the timer incremented. Boundary conditions for the new time step are read and local jam stability checked. The coefficient matrix is then computed and solved, tolerances are checked, and variables are reset for the next time step. For special cases when the entire ice cover is deemed stable, the solution reverts to only the water variables, as the ice variables will remain constant. In this case, an abbreviated coefficient matrix is developed and solved that contains only two equations per reach and the two water boundary condition equations (water discharge upstream and water depth downstream). This abbreviated matrix is a banded symmetrical matrix with two bands above and two below the main diagonal. The reversion to the abbreviated coefficient matrix reduces computation time.

\section{Loosely coupled solution}

The loosely coupled mode of solution also uses the full equations of mass and momentum conservation for the water and ice, including the simplifications for sections and reaches that are determined to be stable. However, in this mode, the water variables are solved separately and sequentially from the ice variables. Both solutions are by the Newton-Raphson iteration scheme presented above. The coefficient matrix for each solution is a banded symmetrical matrix with two bands 


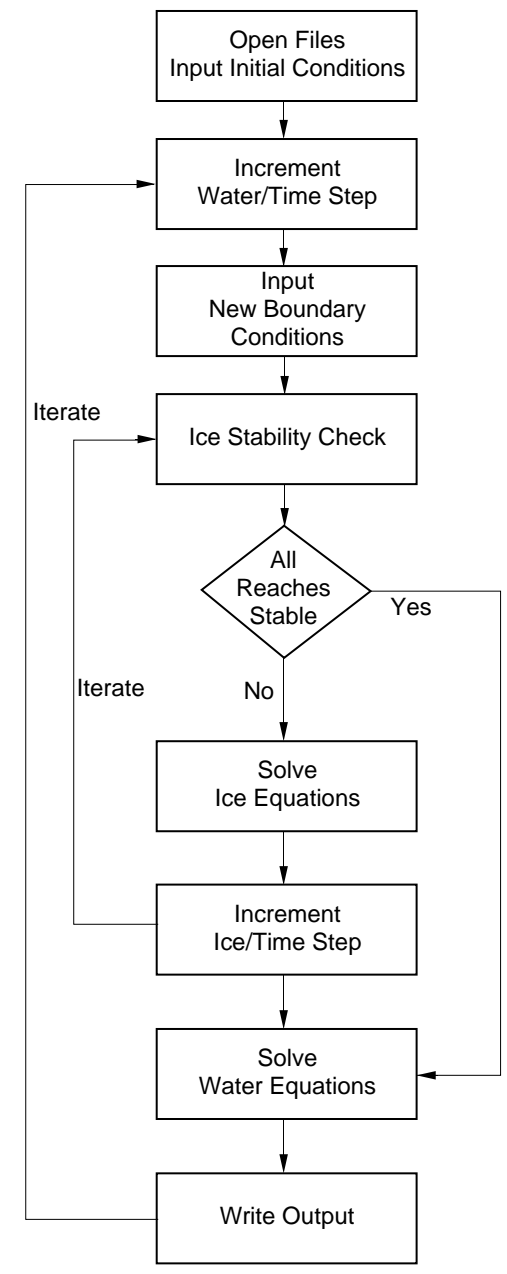

Figure 34. Block diagram for the loosely coupled solution scheme.

above and two below the main diagonal. Two equations for each reach relate the $2 N$ (water or ice) unknowns. A single boundary condition at the upstream and the downstream ends is required to close the system.

The block diagram for the loosely coupled solution scheme is presented in Figure 34. The initial conditions are read, the timer incremented, and boundary conditions for the new time step obtained. The ice variable solution can have a different time step than the water variable solution and is specified as an even fraction of the water time step. This procedure is needed because the ice variables can change faster than the water variables, especially when moving ice stops and thickens. The ice cover stability is checked, the ice variable coefficient matrix is computed and solved, tolerances are checked, and the ice variables are reset for the next ice time step. If the entire ice cover is found stable, the ice variables are simply reset and the ice time step incremented. Following the correct number of ice time step calculations, the water coefficient matrix is computed and solved, tolerances checked, and the water variables reset. This solution mode is included as a comparison to the fully coupled mode because there are currently no existing formulations that are fully coupled. 
Static-unsteady thickness solution

This solution mode is the same technique used by several models where the unsteady water flow is solved, followed by a calculation of the ice thickness. In this mode, the water coefficient matrix is solved and then the ice thickness calculated by an abbreviated form of the conservation of ice momentum equation, in which ice velocity is always assumed to be zero. The resulting calculation of ice thickness may violate ice mass conservation, since the ice velocity is neglected and is an instantaneous (not time-dependent) reassessment of ice thickness. Equation 129 is modified to

$$
\begin{aligned}
& \left(g\left(1-s_{\mathrm{i}}\right) K_{\mathrm{p}}(1-p)+g s_{\mathrm{i}}\right)\left[\frac{\left(\eta_{\mathrm{j}+1}^{n+1}-\eta_{\mathrm{j}}^{n+1}\right)}{\Delta x}\right]+g\left[\frac{\left(d_{\mathrm{j}+1}^{n+1}-d_{\mathrm{j}}^{n+1}\right)}{\Delta x}\right]-g S_{\mathrm{o}} \\
& +\frac{g\left(1-s_{\mathrm{i}}\right) k_{0} \lambda K_{\mathrm{p}}(1-p) \bar{\eta}}{B}-\frac{f_{\mathrm{i}}}{8 s_{\mathrm{i}} \bar{\eta}} \overline{u^{2}}=0
\end{aligned}
$$

where the average (bar) terms are given as simple $x$-dependent averages. Previous formulations for jam thickness by a relation similar in form to eq 150 indicate that the integration can move either in an upstream or downstream direction (Beltaos 1993). This study found, however, well behaved solutions only for integration in the downstream direction, given a thickness relation for the upstream boundary. An equilibrium thickness condition is specified at the upstream boundary by setting the $\partial / \partial x$ terms of eq 150 to zero and solving the resulting relation for $\eta$. In recognizing that the thickness does not decrease upon a reduction in the forces acting on the cover, the newly calculated thickness is compared to the previous thickness and the greater value adopted as the new thickness. This solution mode is the least computationally intensive but neglects the effects of ice momentum. It is included in this study only for comparison to the loosely coupled and fully coupled modes.

\section{Boundary conditions}

Boundary condition equations are necessary for the closure of the system of equations for each solution technique described above. In general terms, upstream and downstream boundary conditions are specified in each case. For the fully coupled solution mode, ice and water boundary conditions are necessary at the upstream and downstream boundaries. Similar to open-water modeling, the water boundary conditions are typically a specified water discharge at the upstream and a specified depth relation at the downstream boundaries. The ice boundary conditions include ice thickness at the upstream boundary and ice velocity at the downstream boundary. As mentioned in the foregoing sections describing the various solution techniques, various relations can be used to describe these specified boundary conditions. 


\section{NUMERICAL MODEL DESCRIPTION}

\section{Model description and capabilities}

A numerical model, called THKFUL, was developed on the basis of the onedimensional, fully coupled equations presented in the Formulation section. The model, written in the FORTRAN programming language, computes the unsteady water depth, velocity, ice thickness, and ice velocity for a river channel. The current version of THKFUL assumes a prismatic, rectangular channel with uniform bed slope, constant values of bed and ice roughness, and invariant and uniform ice properties.

The model THKFUL calls on many subprograms to do various computational tasks. A file utility subprogram opens all required input and output files. Another subprogram reads all the channel geometry, ice property, and physical constant data. One subprogram reads the initial conditions file of depth, velocity, ice thickness, and ice velocity at each cross section. Another reads the new boundary condition data at the beginning of each time step. The stability of the ice cover at each cross section, assessed by means of a force accumulation, is determined using another subprogram. One of two equation-system solvers is called to compute flow and jam variables. FULSOL fills the coefficient matrix using the technique described in the Formulation section for the simultaneous solution of the four dependent variables. However, if the entire ice cover is found to be stable, only the water variables are calculated by WATUNC, which fills and solves a smaller coefficient matrix. Another subprogram writes data to three output files with user-definable write intervals. The first output file provides a listing of the depth, velocity, ice thickness, and ice velocity at each cross section at the specified print interval. The second output file provides profile data for the bed, bottom, and top of the ice cover, and water level at the specified print interval. The third output file contains data necessary for compiling animated plots of the profiles with time. A full program listing can be found in Zufelt and Ettema (1996).

Another program, called UNCTHK, is very similar to THKFUL, but operates in a loosely coupled mode. The ice and water variables are calculated separately and sequentially. In this model, the ice variable solver, ICEUNC, is called one or more times (as specified by the user) for each time that the water solver, WATUNC, is called. This modification was made because the ice variables can change rather abruptly, e.g., when a moving ice cover stops and thickens. The water variables tend to respond slower and smoother. One would expect that the results obtained from the loosely coupled and fully coupled models would approach each other as the time step is reduced. A program listing for UNCTHK is also included in Zufelt and Ettema (1996).

The remainder of this section demonstrates the capabilities of the model THKFUL with sample output plots for a "baseline" configuration of geometric, hydraulic, and ice characteristics. The robustness of the calculation technique is demonstrated and the results of sensitivity testing with relation to Courant number and theta weighting factors for the water and ice are presented. Alternate boundary conditions are described and the effects of using variable size length steps are addressed.

\section{Baseline runs}

A baseline configuration was developed to provide a standard against which future runs could be compared. Care was taken in developing this 5-km-long "hypothetical river" to make sure that the geometric, hydraulic, and ice character 
Table 1. List of baseline testing parameters.

\begin{tabular}{ll}
\hline Number of cross sections & 101 \\
Length step & $100 \mathrm{~m}$ \\
Time step & $30 \mathrm{~s}$ \\
Channel width & $100 \mathrm{~m}$ \\
Bed slope & 0.0005 \\
Solid ice specific gravity & 0.92 \\
Ice cover porosity & 0.40 \\
Darcy-Weisbach friction factor-bed & 0.08 \\
Darcy-Weisbach friction factor-ice cover & 0.12 \\
$k_{0}$-coefficient of lateral pressure & 0.50 \\
$\lambda$-coefficient of friction, ice-on-ice & 0.65 \\
$K_{\mathrm{p}}$-passive pressure coefficient & 3.85 \\
Theta weighting factor-water $(\theta)$ & 0.60 \\
Theta weighting factor-ice $\left(\theta_{\mathrm{i}}\right)$ & 0.60 \\
Maximum number of iterations $/$ time step & 4 \\
\hline
\end{tabular}

ues and eq 27 leads to $k_{0} \lambda=0.325$. By substitution, the values in Table 1 for these two parameters were chosen.

In addition to the parameters in Table 1, the tolerance for each variable, as expressed in eq 147, was set to $10^{-4} \mathrm{~m}$. The upstream boundary conditions were specified as water discharge and equilibrium jam thickness by eq 147 with the nonuniform terms set to zero. The downstream boundary conditions were specified as normal depth beneath a uniformly thick ice cover and zero ice velocity. The upstream water discharge for the baseline testing begins at a steady value of 100 $\mathrm{m}^{3} / \mathrm{s}$ for 20 minutes, rises to $200 \mathrm{~m}^{3} / \mathrm{s}$ during the next 20 minutes, and remains at that level for a total test time of 600 minutes. This upstream discharge hydrograph is shown in Figure 35. The maximum number of iterations of the Newton-Raphson solution scheme per time step was set at four. However, rarely more than two iterations were required to reach the specified tolerance.

The initial conditions for the baseline runs were determined by running the model with a steady upstream water discharge of $100 \mathrm{~m}^{3} / \mathrm{s}$ and a prescribed uniform thickness jam that was significantly greater than the equilibrium thickness predicted from eq 25 to ensure that there would be no further thickening as the water depths and velocities adjusted to their steady, uniform values. Once the initial conditions for water depth and velocity were determined, the initial uniform ice thickness for the baseline testing was chosen to be only slightly greater than the equilibrium value calculated using eq 25 . For the baseline runs, this procedure resulted in the following set of initial uniform values of water depth and velocity, ice thickness, and ice velocity: $1.729 \mathrm{~m}, 0.578$ $\mathrm{m} / \mathrm{s}, 1.50 \mathrm{~m}$ and $0 \mathrm{~m} / \mathrm{s}$, respectively. The equilibrium jam thickness was calculated to be $1.47 \mathrm{~m}$ from eq 25 .

One of the most difficult tasks of modeling jam shoving and thickening is the presentation of results. The process is highly unsteady, with large variations in the values of the dependent variables over fairly short periods. Also, considering the interest in all four of the variables mentioned above means that a

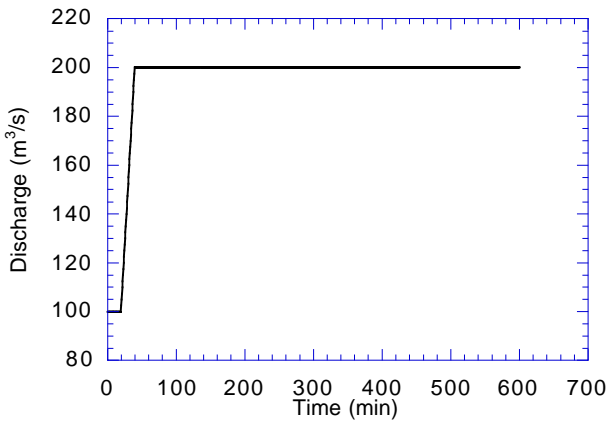

Figure 35. Upstream water discharge hydrograph for baseline run. 
single plot of them becomes overly cumbersome to interpret. The interaction of the dependent variables with and on each other necessitates a form of output illuminating the changes in all the variables with time. One possible format is to present the output in the form of profile plots at specific times. While these sequential time plots can be animated with additional graphic enhancements, they cannot be presented easily in text form. Also, as the length of the modeled system increases, changes of values in the vertical become less obvious because of loss in resolution. Therefore, the water variables (depth and velocity) are plotted on one set of axes directly above the ice variables' (thickness and velocity) axes. This is done for several times at points on the inflow hydrograph where there are significant changes. Figure 36 presents the results of the baseline model for several time slices.

Many interesting observations can be made from Figure 36. By 25 minutes (5 minutes after the initial increase in upstream discharge), the water velocity has increased near the upstream end, but the depth has not changed significantly. The jam is thickening somewhat near the upstream end, but the ice velocity shows the most significant change. As the ice moves, the shear stress between the ice and the water decreases, resulting in less resistance to the water flow and lower water levels. At 35 minutes, ice is moving throughout the system and is by no means uniform. The ice velocity plot shows that the jam failure is not simply a progressive or complete failure as envisioned in the laboratory experiments, but is rather a combination of these two failure modes, with several areas of instability arising within the jam. Each of these instabilities moves downstream with time, resulting in local areas of thickening where ice velocity decreases. The stopping and starting of the ice movement, coupled with the thickening, results in changing stress levels and local states of stability of the jam. The local instabilities move downstream, eventually dying out. At 45 minutes, the ice at the upstream end of the system has ceased moving, while several instabilities are continuing to move downstream. By 100 minutes, all ice motion has stopped and the final thickness profile prevails. The water depths and velocities continue to adjust, however, until they reach steady values.

What becomes most apparent from the output of the baseline runs is the high degree of interaction between the dependent variables. There is no simple mechanism by which an ice jam fails, moves, and thickens. Shoving and thickening are dominated by unsteadiness and nonuniformity. Ice velocity depends on the forces currently exerted against the jam and, thus, is changing constantly as ice comes into motion and stops. Local changes in ice velocity or thickness cause changes in water shear on the underside of the jam, which then affect the forces on the jam. The final thickness profile also points to the importance of ice momentum. The downstream end of the simulated jam had a boundary condition of zero ice velocity, i.e., no ice momentum at that location. Inspection of the ice momentum equation for the last reach, however, shows that ice thickness at the downstream end is affected by the change in momentum between the last two cross sections. Ice thickness is least at the downstream end, where the effects of ice momentum are least, and is $1.73 \mathrm{~m}$. This thickness is greater than the equilibrium thickness $(\eta=1.70 \mathrm{~m})$ calculated using eq 25, which does not include the effects of ice momentum. The upper reaches of the jam have greater ice velocities (as evident in the plots at 30 and 35 minutes). The effects of arresting ice momentum are clearly reflected by the much higher levels of jam thickness in the upstream reaches. 

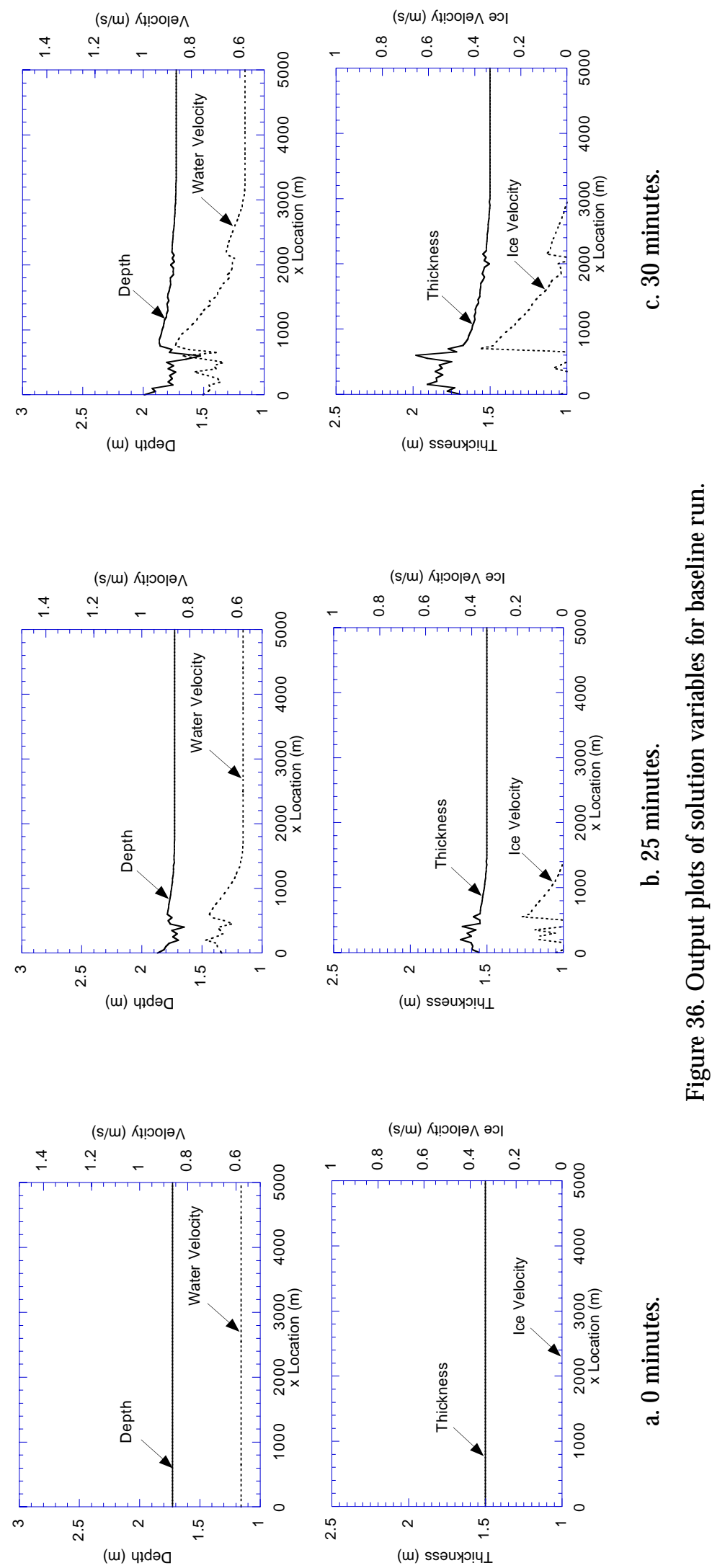

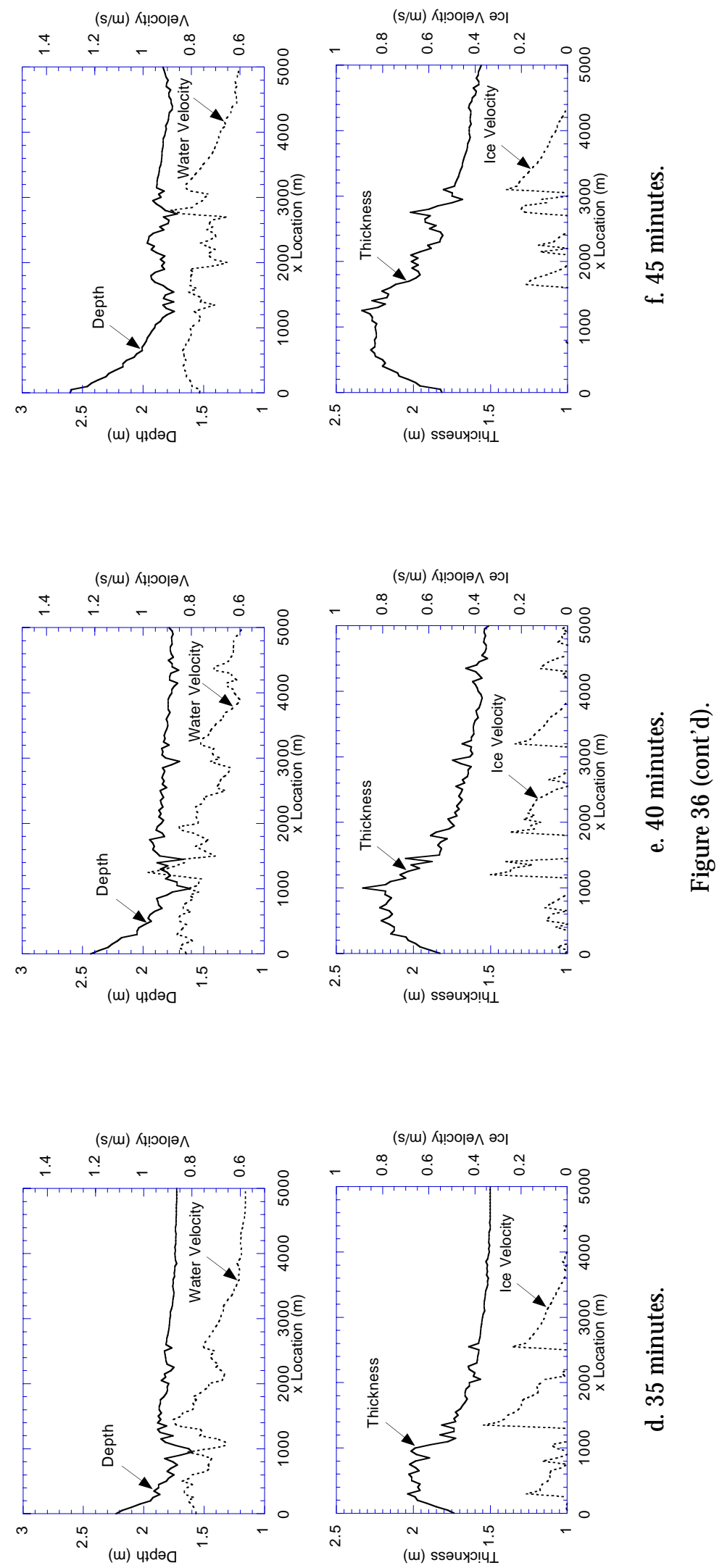

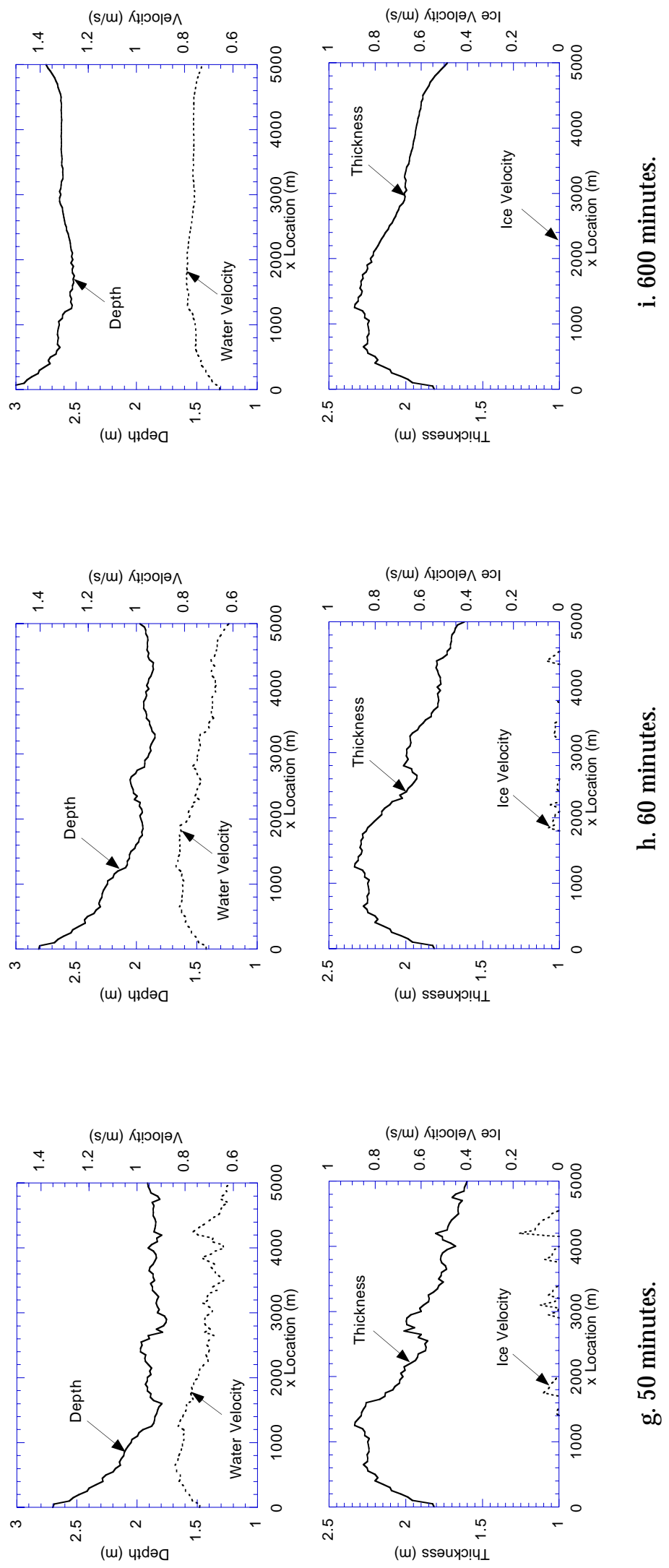


\section{Model rigor}

An unsteady model should be able to operate in steady flow mode without problems. This requirement is demonstrated in the previous section, when steady flow values were obtained for the initial conditions of the variables. A model should also be robust, being able to compensate for initial conditions far from steady or uniform. The model was tested under a variety of "bad input" conditions to see if it could adjust to steady flow conditions.

The first tests involved initial conditions that included either a significant depth increase or decrease. The initial cover thickness was specified such that no thickening would take place. The objective was to see if the model could return to steady conditions of depth and velocity (initial baseline conditions) given these nonuniform initial conditions of depth. The upstream discharge boundary condition was held at $100 \mathrm{~m}^{3} / \mathrm{s}$. Figure 37 shows the water depth and velocity at $0,5,10$, 20, and 120 minutes for an initial local increase in depth. By 5 minutes, the initial water storage "lump" is flattened by gravitational forces acting in the upstream and downstream direction. It then slowly "drains" through the downstream end of the system with time, reaching steady uniform flow conditions by 400 minutes. The water velocity depicted at time 0 is that associated with the onset of the simulation and not the initial conditions. The ice thickness remained constant at the initial value, and hence ice velocity remained equal to zero throughout the run. Figure 38 gives the initial and final water surface level profiles for this run, showing the impossible nature of the initial water levels. A second, similar run looked at a local decrease in depth. Figure 39 shows the water depth and velocity at $0,5,10,20$, and 120 minutes. In this run, the initial water storage deficit sends negative gravity waves both in the upstream and downstream directions. The deficit slowly fills with time, reaching steady, uniform flow conditions by 400 minutes. Figure 40 shows the initial and final water-surface level profiles for this run.

Another run was made using initial ice jam thicknesses that would result in impossible water levels. For this run, the steady, uniform depth of the baseline condition $(1.73 \mathrm{~m}$ at a steady flow rate of 100
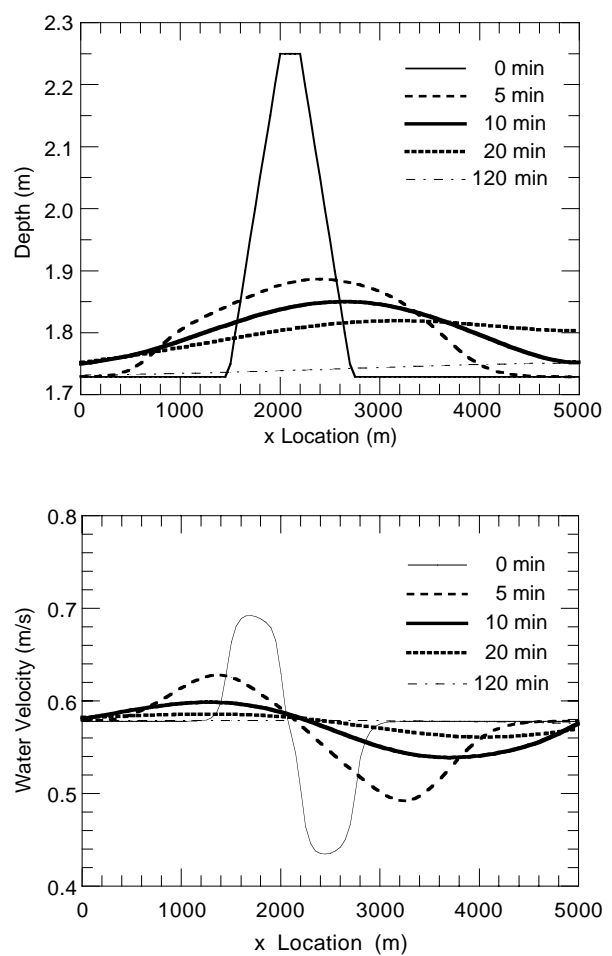

Figure 37. Water depth and velocity profiles at various times for the initial condition of local depth increase.

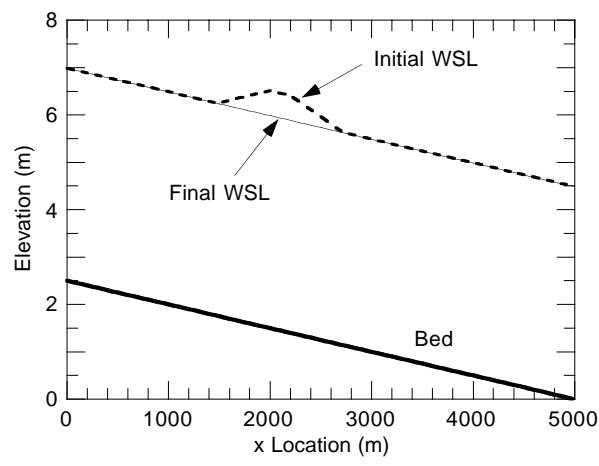

Figure 38. Initial and final water surface level profiles for depth increase. 


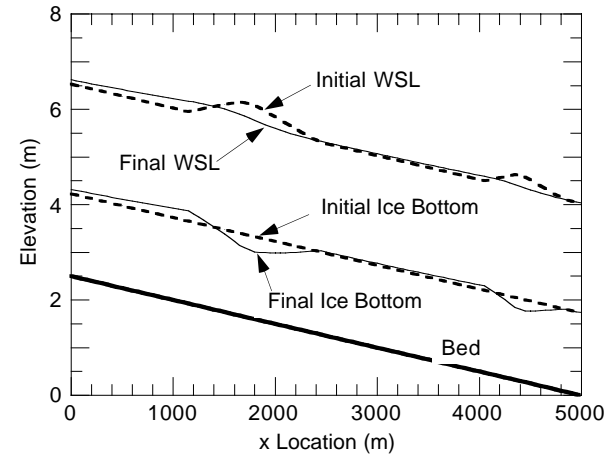

Figure 41. Initial and final water surface level and bottom of ice profiles for an ice jam having nonuniform thickness.

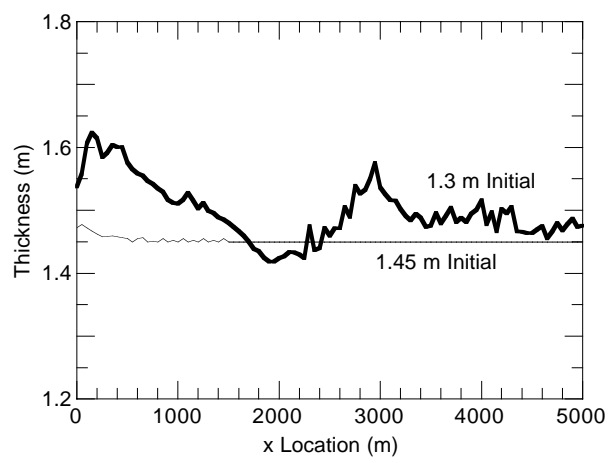

Figure 42. Final jam thickness profiles for two initially unstable jam thicknesses. where greater than the equilibrium jam thickness calculated using eq 25 .

\section{Courant number sensitivity}

Many calculation schemes are sensitive to the computational time and space intervals $(\Delta t, \Delta x)$ used. The sensitivities for hyperbolic problems are usually discussed in terms of the Courant number

$$
C r=\frac{\Delta t|u+c|}{\Delta x}
$$

where $c$ is the gravity wave speed through the fluid. Because of the assumption that ice floats on water and is free to move up and down in the vertical direction in accordance with the dictates of buoyancy, gravity wave speed beneath a jam would be equivalent to that of open water, i.e.

$$
c=\sqrt{g d} .
$$

The Courant number expresses the ratio of the distance traveled by a disturbance in one time step to the length of a computational distance step. For the

simplest Method of Characteristics, the Courant number must be less than or equal to unity so as to ensure that the solution remains within the computational domain. Implicit finite difference methods, as well as interpolated-grid Methods of Characteristics, relax this requirement somewhat. Care must be taken, however, to make sure that disturbances do not travel too far over a time step, thereby resulting in loss of resolution or too much smoothing. For a given computational length step, larger Courant numbers imply larger time steps and less total computation time, with concomitant loss of temporal resolution.

Equations 151 and 152, applied to the baseline parameters in Table 1 and the initial conditions for depth and water velocity, yield $\mathrm{Cr}=2.82$. By calling this value nominally $\mathrm{Cr}=3$, runs were then made with $\mathrm{Cr}=1,5,10,12$, and 24. The Courant number was varied in these tests by adjusting the time step and leaving the length step at $50 \mathrm{~m}$. The boundary conditions file was modified, however, to retain the same shape and timing of the inflow hydrograph. Figure 43 shows the final jam thickness profile for the runs with $C r=1,3$ (standard), 5, 10, 12, and 24. The plot shows that there is little difference in results with $\mathrm{Cr}$ values up to 12 , and that some diffusion or smoothing is evident for $\mathrm{Cr}=24$.

\section{Theta-weighting factor analysis}

The theta-weighting factors for the water $\theta$ and for the ice $\theta_{\mathrm{i}}$ are presented in the Discretization of the System of Equations section and are used to describe the time averaging between the known conditions at the current time step and the future, 
calculated conditions. From eq $118 \mathrm{~b}$ and 119 , it can be seen that, if $\theta=1.0$, there is no consideration of previous values of the variables or their derivatives in the solution for their values at the new time step (except in the time derivative). Cunge et al. (1980) show that while the Preissmann scheme becomes secondorder accurate under the special case of $\theta=0.5$, it also becomes dispersive due to the finite difference approximation of some of the higher order terms. For openwater simulations, the dispersion results in a wavy surface with relatively highfrequency oscillations. By specifying $0.5<\theta \leq 1.0$, the solution becomes diffusive, smoothing out the high-frequency oscillations and providing a more realistic solution. The ideal solution would be one in which dispersion and diffusion are both minimal.

A separate weighting factor $\theta_{i}$ was specified for the ice. The movement of the ice can be quite different from that of the water because it can start and stop rather suddenly. The effects of using the current values of ice velocity to calculate

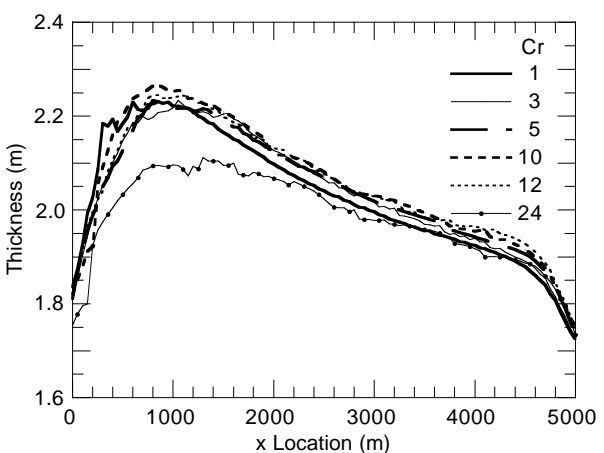

Figure 43. Final jam thickness profiles for Courant numbers of 1, 3, 5, 10, 12, and 24.

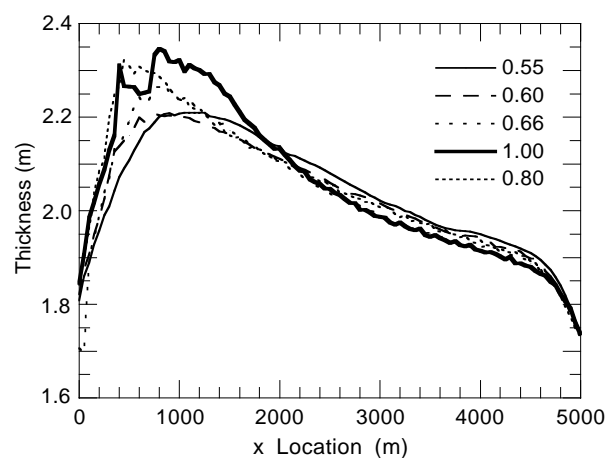

Figure 44. Final jam thickness profiles for $\theta_{i}$ $=1.0$ and $\theta=0.55,0.6,0.66,0.8$, and 1.0. the new values were unknown. For example, if the ice cover was in motion and the new stability check showed that it should be stable (the new ice velocity, $v=0$ ), the previous value of ice velocity may have an undesirable effect on the newly calculated ice thickness if $\theta_{i}<1.0$. Detailed inspection of the calculated ice velocities (as ice begins moving and as it slows to a stop), however, showed that absolute changes in ice velocity over a time step were not large and, thus, the $\theta_{\mathrm{i}}$-weighted average of ice velocity would provide a proper solution.

The effects of the water-weighting factor on the solution were investigated by running the baseline conditions at a variety of $\theta$ values for $\theta_{i}=1.0$. Values of $\theta$ tested included $0.5,0.55,0.6,0.66,0.8$, and 1.0. The simulation with $\theta=0.5$ resulted in unacceptable oscillations in the water depth and velocity, which, because the solution is fully coupled, resulted in large oscillations in ice velocity and thickness. The oscillations in the water variables aggravated the oscillations in the ice variables and the solution became highly unstable. For instance, the depth would decrease with an accompanying water velocity increase that increased ice velocity and thickening, thereby further reducing flow depth. Figure 44 shows the final jam thickness profile for $\theta=0.55,0.6,0.66,0.8$, and 1.0. While $\theta=0.55$ shows a smooth final profile, it resulted in highly variable water depths, especially at the initial increase in upstream water discharge. A value of $\theta=0.6$ provided the most acceptable solution in terms of both water depth and ice thickness throughout the simulation period. 


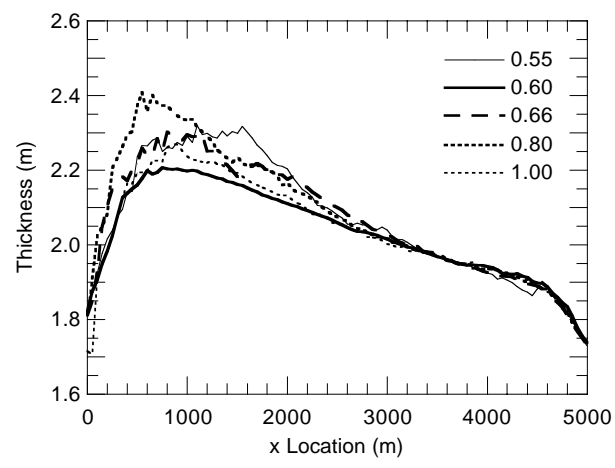

Figure 45. Final jam thickness profiles for $\theta=0.6$ and $\theta_{i}=0.55,0.6,0.66,0.8$, and 1.0.

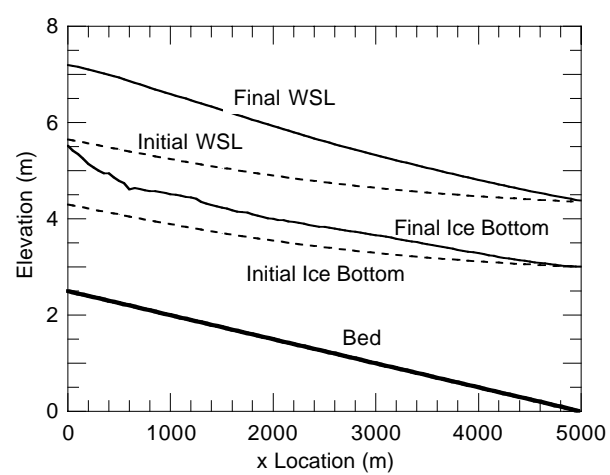

Figure 46. Final bed, bottom of jam, and water surface level profiles for the condition of downstream depth being held at $3.0 \mathrm{~m}$.
The effects of the ice-weighting factor were then investigated by running the baseline conditions with $\theta=0.6$ and $\theta_{\mathrm{i}}=0.5,0.55,0.6,0.66$, and 0.80. Again, with a value of $\theta_{\mathrm{i}}=0.5$, the solution became highly unstable and gave unreasonable results in terms of ice thickness. Figure 45 shows the final jam thickness profile for $\theta_{i}=0.55,0.6,0.66,0.8$, and 1.0. The profile shows little variation with $\theta_{i}=0.6$, providing physically reasonable results. Inspection of the values of the other dependent variables throughout the simulations showed that either $\theta_{i}=$ 0.6 or 0.66 would be acceptable. For the remainder of the model testing, the values of $\theta=0.6$ and $\theta_{i}=0.6$ were used.

\section{Alternate boundary conditions}

The boundary conditions for the baseline runs included specified water discharge and equilibrium thickness at the upstream end, and zero ice velocity and a condition of normal flow depth beneath the jam at the downstream end. While these boundary conditions represent plausible natural conditions, they are by no means all inclusive. A dam, for instance, typically has a water surface slope that decreases in the downstream direction and results in downstream water levels that are significantly above the normal depth. Ice jams in dam pools often are resistant to shoving and thickening because of the reduced shear stress and gravity forces exerted on them. The equilibrium jam thickness at the upstream end also implies that there is an unlimited supply of ice upstream of the jam that would continually move into the modeled reaches at the equilibrium thickness.

Two alternative boundary condition types were developed. A condition of specified ice thickness at the upstream end of the jam facilitates simulation of the upper transition zone where jam thickness is reduced. The effects of lower upstream jam thickness on the shape of the final jam thickness profile can also be investigated. A condition of specified water depth at the downstream end of the system facilitates simulation of ice jams in reservoirs or at other water-elevation control structures.

A run was carried out to simulate jam shoving and thickening in a reservoir where the downstream depth was held at $3.0 \mathrm{~m}$. The initial water depths and velocities for this run were determined by running a steady water discharge of 100 $\mathrm{m}^{3} / \mathrm{s}$ with a uniform depth throughout the $3.0 \mathrm{~m}$ and letting the system attain steady flow conditions. The baseline inflow hydrograph was then run with all other parameters the same as for the baseline test. Figure 46 shows the initial and final water surface, and the bottom profiles of the jams. The downstream end experienced no shoving and thickening, remaining at the initial thickness of $1.45 \mathrm{~m}$, while 
the upstream reaches thickened to over $2.0 \mathrm{~m}$. The initial jam thickness profile for this test was specified as a uniform $1.5 \mathrm{~m}$.

Given the water surface profile that was specified as the initial conditions, the initial stable jam thickness would have been less in the downstream reaches. This may have resulted in significant shoving and thickening in those areas, leading to a totally different final jam thickness profile.

For another run, the downstream depth was held at the initial uniform depth of $1.729 \mathrm{~m}$. All the other conditions were the same as the baseline run. Figure 47 shows profiles of the bed, bottom of jam, and water-surface level for this run. Confining the downstream depth required that the water velocity increase, which resulted in significant thickening and water-surface level rises. This figure shows that the profiles of the water surface and jam bottom at the downstream end are very similar to the classical downstream transition configuration of an idealized ice jam (e.g., Ashton 1986).

Several other runs were made using a specified jam thickness as the upstream boundary condition. Three runs with jam thicknesses held at 1.47,1.4, and $1.3 \mathrm{~m}$ at

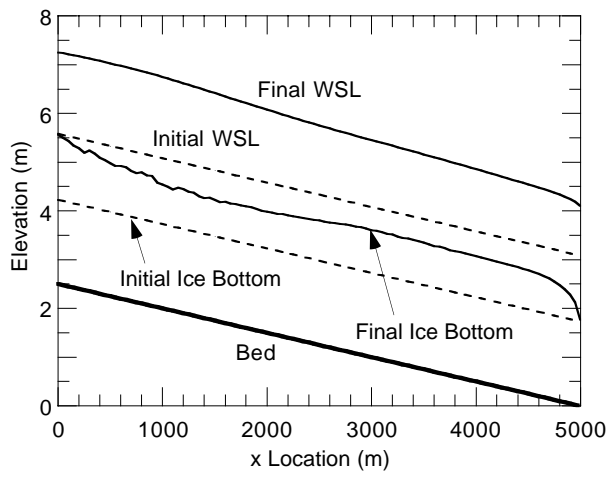

Figure 47. Final bed, bottom of jam, and water-surface level profiles for the condition of downstream depth being held at an initial depth of $1.729 \mathrm{~m}$.

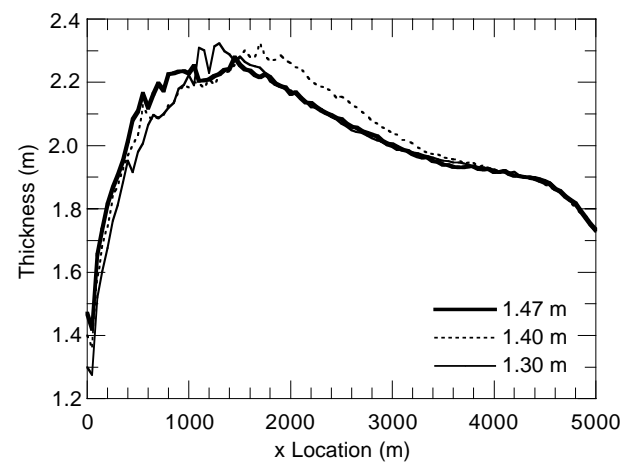

Figure 48. Final jam thickness profiles for upstream jam thickness being held at 1.47, 1.4 , and $1.3 \mathrm{~m}$. the upstream end were compared to the baseline run (equilibrium jam thickness at the upstream end). Figure 48 shows the final jam thickness profiles for these runs. The profiles are very similar, with differences primarily occurring in the most upstream $1000 \mathrm{~m}$. The implicit nature of the numerical solution procedure extends the effect of the specified (artificially low) jam thickness for several computational steps downstream. Also evident from the figure is that the progression of the shoving and thickening differs in each run, resulting in slightly different jam thickness profiles. It would be expected that a locally weak reach would shove and thicken first, setting up changes in the water depth and velocity (since the solution is fully coupled). Minor changes in the water variables translate into minor changes in the ice variables and in the final, stable jam thickness profiles.

\section{Effects of variable length steps}

Final testing of the model involved examining the effects on the predicted results of variable computational length steps. The finite-difference formulation allows variable length steps, whereas other computation methods do not without considerable difficulties (i.e., the Method of Characteristics). This facility is needed because field data are often variable in the distance between cross sections. While the computational effects of variable length steps should be minimal, care must be 


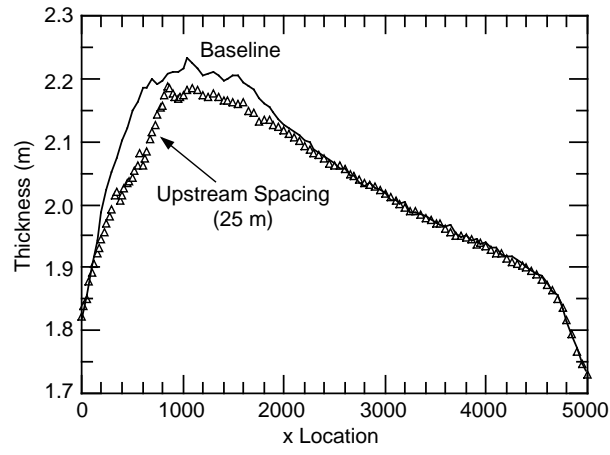

a. In the first $1000 \mathrm{~m}$ of the system.

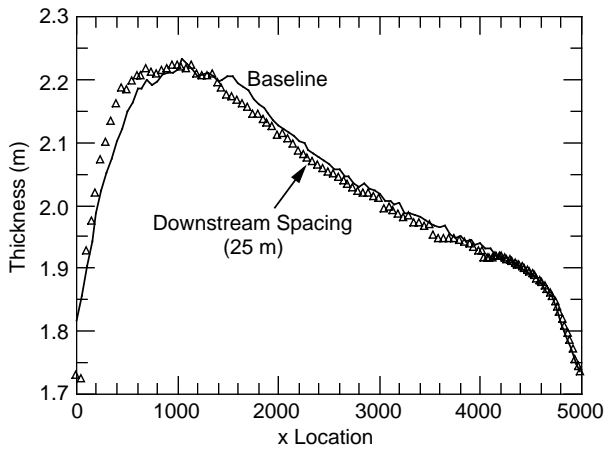

b. In the last $1000 \mathrm{~m}$ of the system.

Figure 49. Final jam thickness profile for length step reduced to $25 \mathrm{~m}$.

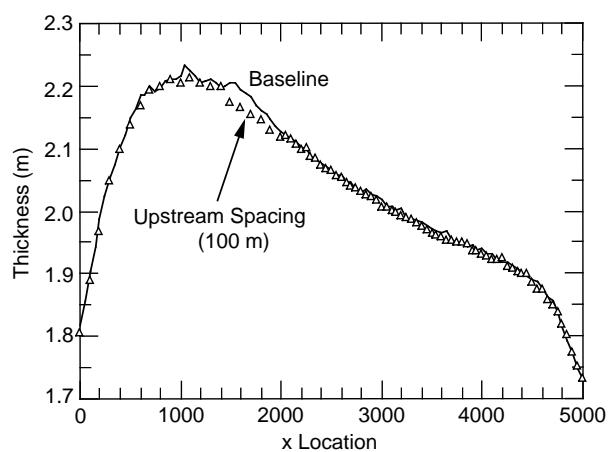

a. Length step increased to $100 \mathrm{~m}$.

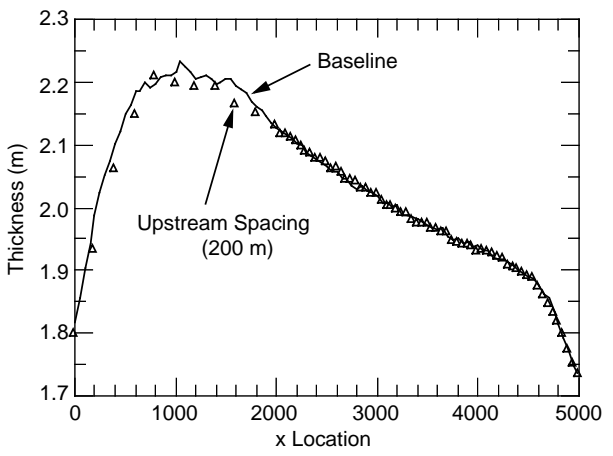

b. Length step increased to $200 \mathrm{~m}$.

Figure 50. Final jam thickness profile for length step changes in the first $2000 \mathrm{~m}$ of the system.

taken not to violate any Courant number limitations identified earlier.

The baseline run had uniform length steps of $50 \mathrm{~m}$, which give $\mathrm{Cr}=3$. The Courant-number sensitivity findings described earlier indicate that $\mathrm{Cr}$ values in the range of 1 to 12 should provide acceptable results. From eq 151, this finding translates to acceptable length steps ranging from 16.7 to $200 \mathrm{~m}$. Several runs were made with a variety of length steps.

The first run involved changing the length step to $25 \mathrm{~m}$ for the first $1000 \mathrm{~m}$ of the system. This step length decreased the $\mathrm{Cr}$ to 1.5 in the first $1000 \mathrm{~m}$ of the system and increased the total number of cross sections to 121. All the other conditions were the same as for the baseline run. A similar run decreased the length step to 25 $\mathrm{m}$ for the most downstream $1000 \mathrm{~m}$ of the system. These two reaches are where the greatest variations in the final jam thickness profile exist. Figure 49 presents the final jam thickness profiles for these two runs, compared to that of the baseline run. The 25-m upstream spacing resulted in a more gradual rise to the maximum thickness, which occurred around $1000 \mathrm{~m}$. The final jam thickness was up to $0.05 \mathrm{~m}$ less than the baseline run, but only for locations upstream of $2000 \mathrm{~m}$. Almost no difference existed between the baseline and the 25-m downstream spacing runs, especially in the most downstream $1000 \mathrm{~m}$ where the spacing was changed.

The next two runs increased the length step in the most upstream $2000 \mathrm{~m}$ of the system to 100 and $200 \mathrm{~m}$ respectively. The values of $\mathrm{Cr}$ for the upstream $2000 \mathrm{~m}$ increased to 6 and 12, with the total number of cross sections reduced to 81 and 71 respectively. Figure 50 presents the final jam thickness profiles for these two tests, 


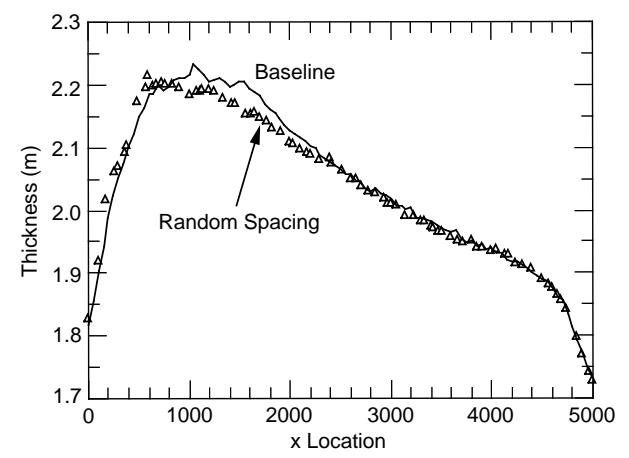

Figure 51. Final jam thickness profile for random length steps throughout the system. plotted with the jam thickness profile for the baseline test. The 100-m upstream spacing resulted in a final thickness profile that was almost identical to the baseline run. The profile shape and maximum thickness were very similar. The 200-m upstream spacing showed slightly more variation than the $100-\mathrm{m}$ upstream spacing but was also very similar in shape and maximum thickness to the baseline run.

The final run simulated random spacing of cross sections (typical of field data) by using randomly assigned length steps between 25 and $100 \mathrm{~m}$ (intervals of $5 \mathrm{~m}$ ) for a total flow length of $5000 \mathrm{~m}$ with 81 cross sections. Now, $\mathrm{Cr}$ ranged from 1.5 to 6 . Figure 51 shows the final jam thickness profile for this run along with the baseline results. Minor variations existed in the final thickness profile (up to $0.03 \mathrm{~m}$ ), primarily in the most upstream $2000 \mathrm{~m}$ of the system. Overall profile shape and maximum thickness, however, were very similar to the baseline run. This check on model robustness shows that variable length steps have minor negligible effects on predicted results, as long as the Courant number limitations identified earlier are followed.

\section{Summary}

The main points emerging here are that the fully coupled model of ice jam dynamics is robust and versatile. Alternate boundary conditions and the use of variable length steps make the model adaptable to a variety of physical situations and computational capabilities. The sensitivities of results to computational parameters are found to be minimal as long as the parameters are held within reasonable (practical, physically based) ranges.

Even at this stage of use, the model shows that the effects of ice momentum on the solution are quite significant, as evidenced in the profiles of jam thickness. For example, the baseline jam thickness profile shows that almost the entire system ends up with jam thicknesses greater than those calculated using the stationary jam theory embodied in eq 25. Furthermore, the model shows that shoving and thickening make up a highly unsteady process, marked by variations and interactions between the dependent variables not represented in past modeling efforts. Small changes in any of the dependent variables can result in variations in the timing of the shoving and thickening event, with subsequent variations in the final jam thickness profile.

\section{UNSTEADY JAM DYNAMICS}

\section{Effects of ice momentum}

A primary objective of this study is to ascertain the effects of ice momentum on jam formation and thickness. The importance of ice momentum already became evident when the stability and overall robustness of the numerical model developed for the study were evaluated. The fully coupled, numerical model includes ice momentum in the conservation of momentum expression formulated for ice in 


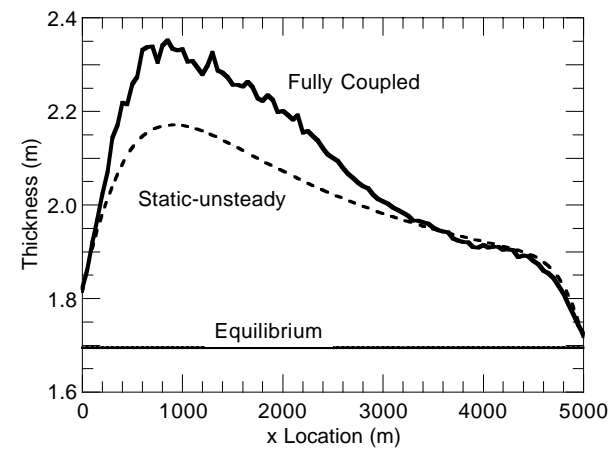

Figure 52. Comparison of thickness profile predicted by static-unsteady thickness model to fully coupled model and equilibrium thickness. a jam. The static-unsteady thickness solution presented earlier allows jam thickness to fluctuate in time and space, but the streamwise movement of the ice, and therefore ice momentum, are not included.

Early formulations of equilibrium jam thickness are based on uniform flow depths and jam thickness in a reach of constant water surface slope. As a result, the force balance only contains the following terms: shear stress on the underside of the jam from the water flow, the downstream component of the gravity force on the jam (based on the bed slope because of the uniform flow assump-

tion), and the shear resistance at the banks. Because the thickness is considered uniform within the equilibrium reach, those formulations contain no terms reflecting changes in hydrostatic pressure, jam strength, water depth, or jam thickness along the reach considered. The formulation leading to eq 150, however, includes these terms, but with the ice velocity terms reduced to zero. The resulting force balance is affected by the nonuniform thickness of the jam as well as the nonuniform depths within a reach. The more complete balance of forces expressed in eq 150 enables more accurate modeling of jam profile, whether the profile includes an equilibrium-thickness section or not.

To show the effects of ice momentum on jam thickness profile, the results of the static-unsteady thickness solution leading to eq 150 were compared to those obtained by the fully coupled model. The loosely coupled moving-ice model was also run for the baseline conditions and compared to the fully coupled results to demonstrate the effects of the coupling of the dependent variables on the jam thickness profile.

The static-unsteady thickness model was run with the baseline inflow hydrograph and all other parameters the same as for the baseline condition. Figure 52 shows the predicted jam thickness profile obtained by the static-unsteady thickness model. Also plotted are the thickness profile predicted using the fully coupled model and the equilibrium thickness for the final flow level of $200 \mathrm{~m}^{3} / \mathrm{s}$. The static-unsteady force balance represented by eq 150 includes the partial derivative terms representing changes in jam strength and water surface slope along a reach. The changing water surface slope becomes particularly important as discharge increases, increasing the downstream-acting forces with a subsequent increase in jam thickness. With a milder rate of increase in discharge, the water surface slope would remain closer to the bed slope, resulting in a static-unsteady thickness profile nearer to the equilibrium thickness. The importance of ice momentum is clearly evident from the fully coupled thickness profile and results in much greater ice thickness. Ice velocity, and, hence, ice momentum, are greatest in the upstream reaches where the increasing discharge wave is steepest. The ultimate arrest of this ice momentum is responsible for the significantly greater thickness.

The effects of fully coupling the solution are determined by comparison with the results from the loosely coupled model. The loosely coupled moving-ice model was run with the inflow hydrograph and all other parameters the same as for the 
baseline condition. The number of ice calculation cycles for each water calculation cycle was set to 1 . Another run was made with the number of ice calculation cycles set to 2 . Figure 53 shows the final jam thickness profiles for the fully coupled and loosely coupled model runs. The fully coupled model results show a more pronounced effect of ice momentum. As the number of ice calculation cycles increases, the resulting thickness profile attains a shape closer to the fully coupled thickness profile. The minimal computational time saved

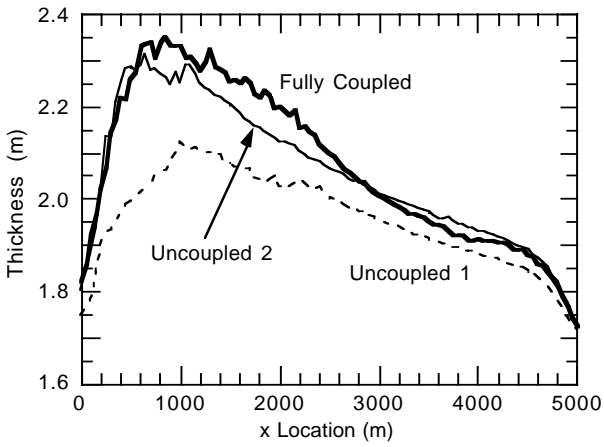

Figure 53. Comparison of thickness profile predicted by loosely coupled model to fully coupled model. by using the loosely coupled model with two or more ice calculation cycles is outweighed by the benefits of using a truly fully coupled model.

\section{Comparison with steady-state models}

Steady-state models of jam thickness existed before high-performance mainframe and personal computers became available. The computational power that exists today allows very large coefficient matrices to be solved with little effort. The execution time of the fully coupled model for tests similar to the baseline configuration described in the Baseline Runs section is much less than the time required for compilation of input files and analysis and plotting of output files. Even though computational speed and capability have greatly increased, there still are reasons (such as unsteady boundary condition information requirements) that a complex model might not be used. For many situations, a steady-state model may be appropriate and provide results within a specified tolerance or accuracy desired. For instance, Uzuner and Kennedy (1976) could formulate the upstream transition region of jam thickness using a frame of reference that traveled upstream at the speed of the progression of the jam front. The moving reference frame made the flow quasi-steady and the computation much easier.

A widely used steady-state model for calculating water levels in ice-covered channels is HEC-2 modified with the ice cover option or with the utility ICETHK, or both. This model has been shown (Zufelt and Doe 1986) to yield accurate results, provided the appropriate values of the jam-related variables are chosen. An important input variable for a steady-state model is water discharge. However, ice jams are markedly unsteady events characterized by widely fluctuating discharges: as level ice covers (whence the jam ice originates) and ice jams fail, releasing stored water, or as jams form, causing local reductions in discharge. A steady-state model cannot account for local variations in discharge caused by jam formation or failure.

The discharge record shown in Figure 54 illustrates how unsteady flows can be during jam breakup. Fortunately, the stage gauge used to estimate the discharges in Figure 54 was located approximately $1 \mathrm{~km}$ downstream of the toe of the jam and was ice-free during the event (except when the jam failed and ice passed downstream). The figure clearly shows when the jam initially formed, evidenced by a drop in the discharge downstream at the gauge. It shows, too, the rise in discharge as the water level in the jammed reach rose, and indicates the ultimate failure of the jam, evidenced by the rapid peak and passage of the discharge wave. The local 


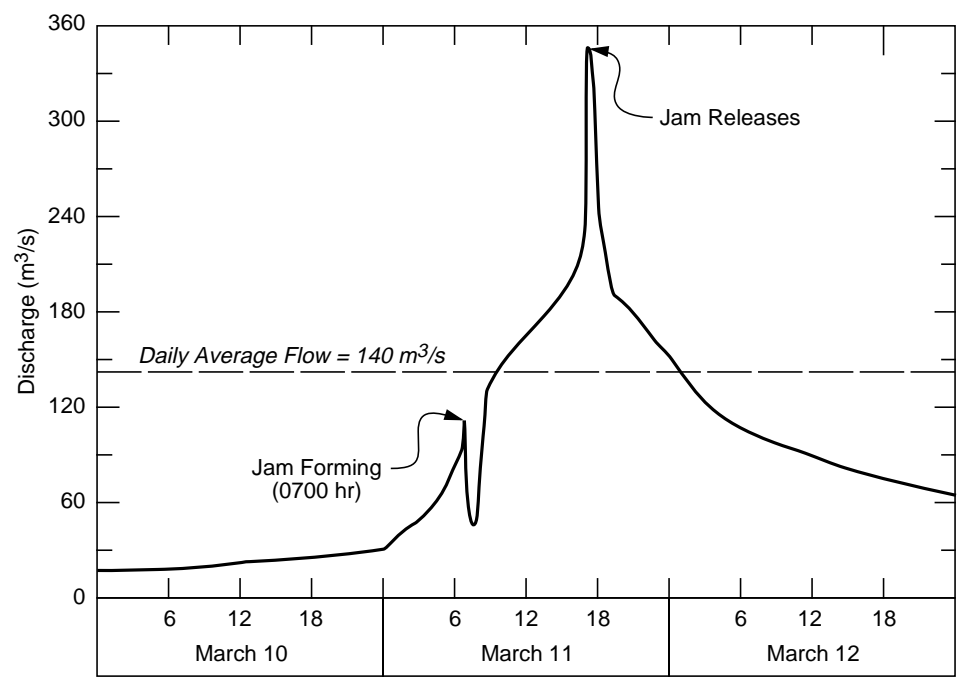

Figure 54. Discharge record during breakup jam initiation and failure.

discharge at upstream locations, however, could be markedly different from that recorded at the gauge. In many cases, gauges are not located near the jammed reach and estimates of discharge become difficult. In some situations, a jam may create a backwater effect on the measuring gauge, and interpretation of the stage recording (in developing the discharge record) also becomes questionable. Winter discharge estimates can be off by as much as $25 \%$ because of backwater from ice jams. As a result, the choice of discharge used in a steady-state model can greatly affect the results of the modeling.

The fully coupled model was used, in conjunction with the static-unsteady thickness model with a constant discharge, to determine how the choice of water discharge affects jam thickness profile. Figure 55 shows a hypothetical breakup jam discharge hydrograph. The hydrograph is characterized by a fast rise and very short duration peak, similar to the hydrograph depicted in Figure 54. While the fully coupled model uses this hydrograph as an upstream boundary condition, a steady-state model requires that a single discharge be chosen as an input variable. One choice might be the peak value of discharge. If the modeled system were of significant length, however, the instantaneous peak would be attenuated as it traveled downstream. Thus, the discharge felt at downstream locations (and consequently shear stress on the underside of the jam) would be less.

Another choice for the steady-state discharge might be a time-averaged discharge based on the time required for a disturbance to pass through the system. Two tests were run with the static-unsteady thickness model at a constant discharge level and corresponding uniform values of depth and water velocity. This simulated the calculations of a steady-state model: one with a constant discharge of the peak value of $200 \mathrm{~m}^{3} / \mathrm{s}$ from Figure 55 and the other with a time-averaged constant discharge value of $167 \mathrm{~m}^{3} / \mathrm{s}$.

Figure 56 shows the final jam thickness profiles for these two runs, along with the final jam thickness profile predicted using the fully coupled model with the unsteady discharge hydrograph. The steady discharge levels and uniform initial conditions result in uniform jam thickness equal to that given by the equilibrium jam formulation represented by eq 25 . The fully coupled thickness profile is greater than the equilibrium thickness for both of the steady discharge choices everywhere, 
except the most downstream reaches. The effects of ice momentum and nonuniformities in depth and thickness on the fully coupled profile are clearly evident, even for this discharge hydrograph with a very short duration peak. The smaller thicknesses at the downstream end are ascribable to attenuation of the peak flow as it travels downstream. Figure 57 shows the discharge hydrograph at the upstream boundary, the midpoint of the modeled system, and the downstream boundary. The peak flow is greatly reduced as it travels downstream, with a maximum value less than $150 \mathrm{~m}^{3} / \mathrm{s}$ by the time it reaches the midpoint of the modeled system. The wave is also significantly flattened. The high-frequency fluctuations in discharge at the mipoint come from ice movement. As the ice moves and stops, resistance to water flow varies, resulting in local fluctuations in water discharge.

\section{Dimensionless momentum parameter}

The numerical experiments show that ice momentum is important in the prediction of jam thickness. Its effect can be a large one, not only in the magnitude of the thickness as compared to that predicted by equilibrium theory, but also in the nonuniform shape of the thickness profile. Some of the experiments show, however, that, for small changes in the discharge or for initial conditions of jam thickness that are slightly less than equilibrium thickness associated with the expected flow levels (e.g., those presented in the Model Rigor section), ice momentum has a negligibly small effect.

For example, the final jam thickness profile in Figure 42 is within $10 \%$ of the equilibrium thickness value for that water flow rate, for the jam with an initial thickness of $1.3 \mathrm{~m}$. The average jam thickness, however, is $1.5 \mathrm{~m}$ or $2 \%$ above the equilibrium value. For this case, a steady-state equilibrium thickness calculation would have proved satisfactory. When unsteady boundary condition data are not available, it may be necessary to use a simpler steady-state model to provide jam thickness estimates, rather than using the fully coupled unsteady model. Therefore, it is useful to identify a parameter, or set of parameters, that indicate when ice 
momentum is important and should be taken into account for determining jam thickness profile. For these cases, the results of simpler steady-state models should be used with caution.

The force balance on a jam at static equilibrium can be represented by equations such as eq 19 or 150 with the nonuniform terms set to zero. The downstream-acting forces of the water shear stress on the jam underside and the component of gravity due to the weight of the jam are balanced by the shear resistance at the banks

$$
\frac{f_{\mathrm{i}} u^{2} B}{8}+g S_{\mathrm{o}} B s_{\mathrm{i}} \eta=g s_{\mathrm{i}}(1-p)\left(1-s_{\mathrm{i}}\right) k_{0} \lambda K_{\mathrm{p}} \eta^{2} .
$$

If the simplified notation of $a+b=c$ is used to describe eq 153, then $a / c$ represents the portion of the jam strength (represented by the bank shear resistance) mobilized by water shear stress. By assuming a wide, rectangular channel, the first term in eq 153 can also be written as

$$
a=\frac{f_{\mathrm{i}} u^{2} B}{8}=f_{\mathrm{i}}\left(\frac{Q S_{\mathrm{o}} g \sqrt{2 B}}{8 f_{\mathrm{o}}}\right)^{2 / 3} .
$$

Together with the equilibrium thickness given by eq 25, Table 2 was generated for jams at the verge of stability (equilibrium thickness) for flow in channels of different bed slopes.

Table 2 shows that, for very low bed slopes, downstream gravity forces are minimal with the water shear stress mobilizing most of the jam's strength. For the smallest bed slope, when the discharge was increased by a factor of $2, a / c$ remained very high and the equilibrium thickness increased by $24 \%$. At the highest bed slope shown, water shear stress only engages a small part of the jam's strength. A similar factor of 2 increase in discharge increases $a / c$, but it still remains low and only a $6 \%$ change results in the equilibrium thickness. Smaller bed slopes are associated with lower water velocities, but also much lower jam thicknesses. As bed slope increases, water shear stress increases, but at a slower rate than the gravity force term ( $b$ in eq 153). Once a jam fails, it is water shear stress that transports the ice downstream. While this finding is not entirely intuitive, it means that ice momentum effects become more important as the $a / c$ ratio increases, i.e., for smaller bed slopes and smoother channels.

Consider further the $a$ and $c$ terms and their ratio in eq 153 for a bed slope of 0.0005 given in Table 2. Equation 154 shows that, for a given discharge, the $a$ term has a set value. Equation 153 balances the forces by adjusting to the value of equilibrium thickness, giving a value for $a / c$ at the limit of stability. But, for example, if the thickness had attained a value greater than the equilibrium level (say $1.60 \mathrm{~m}$ ),

Table 2. Ice parameters for channels at different bed slopes.

\begin{tabular}{lcccc} 
Bed slope, $\mathrm{S}_{o}$ & $\eta_{\text {eq }}$ at $100 \mathrm{~m}^{3} / \mathrm{s}$ & a/c at $100 \mathrm{~m}^{3} / \mathrm{s}$ & $\eta_{\text {eq }}$ at $200 \mathrm{~m}^{3} / \mathrm{s}$ & a/c at $200 \mathrm{~m}^{3} / \mathrm{s}$ \\
\hline 0.000005 & $0.21 \mathrm{~m}$ & 0.960 & $0.26 \mathrm{~m}$ & 0.968 \\
0.00005 & $0.49 \mathrm{~m}$ & 0.829 & $0.60 \mathrm{~m}$ & 0.861 \\
0.0005 & $1.47 \mathrm{~m}$ & 0.432 & $1.69 \mathrm{~m}$ & 0.509 \\
0.001 & $2.30 \mathrm{~m}$ & 0.277 & $2.57 \mathrm{~m}$ & 0.352 \\
0.0025 & $4.74 \mathrm{~m}$ & 0.121 & $5.02 \mathrm{~m}$ & 0.171 \\
\hline
\end{tabular}


eq 153 would no longer be in balance and would represent a condition of greater jam stability. In this case, the $a / c$ ratio decreases because the $a$ term remains constant. As water discharge increases, the jam should remain stable for a longer period prior to shoving and thickening, thus resulting in lower ice velocities and less of an effect of ice momentum on the jam thickness. Also, for a given initial jam thickness (whether at the limit of stability or not), a smaller discharge rise has less effect on jam thickness than does a larger one. Finally, a larger relative discharge increase has more effect than does a smaller one. For instance, ice momentum would be expected to influence the final jam thickness more for an increase from 100 to $200 \mathrm{~m}^{3} / \mathrm{s}$ than an increase from 200 to $300 \mathrm{~m}^{3} / \mathrm{s}$.

To express these trends, a dimensionless parameter was developed that includes initial jam conditions (indicating how close the jam is to the limit of stability), as well as the relative increase in discharge expected. This number is

$$
\Omega=\left(\frac{a}{c}\right)\left(\frac{\Delta Q}{Q_{\text {in }}}\right)=\left(\frac{f_{\mathrm{i}} u^{2} B}{8 g s_{\mathrm{i}}(1-p)\left(1-s_{\mathrm{i}}\right) k_{0} \lambda K_{\mathrm{p}} \eta^{2}}\right)\left(\frac{\Delta Q}{Q_{\text {in }}}\right)
$$

where $Q_{\text {in }}$ is initial water discharge and $\Delta Q$ is the expected change in discharge. This dimensionless parameter is the product of the initial state of stability of the jam and the relative discharge increase applied to cause an instability.

Several runs were made with the fully coupled model using an inflow hydrograph that rose at the same rate as the baseline inflow hydrograph, but with ten different combinations of initial discharge and discharge increase as listed in Table 3.

Runs were made for eight different bed slopes of $0.00005,0.00008,0.0001,0.00025$, $0.0005,0.00075,0.001$, and 0.0025 . The final jam thickness profile for each of these runs was compared to the equilibrium jam thickness $\eta_{\text {eq }}$ for the final discharge as calculated by eq 25 . Average values of jam thickness $\bar{\eta}$ were calculated for each profile. The values of $\Omega$ were plotted against $\bar{\eta} / \eta_{\text {eq }}$ in Figure 58. The data points delineate a line, showing combinations of channel and flow conditions, or $\Omega$ values, for which ice momentum significantly affects jam thickness (from the $\bar{\eta} / \eta_{\text {eq }}$ value). It is clear that ice momentum is very important for low bed slope values, because water shear stress engages the greater portion of the jam strength for these cases. The scatter in the data at higher values of $\bar{\eta} / \eta_{\text {eq }}$ is most likely attributable to the highly nonuniform jam thickness profiles for those cases. Figure 59 shows a plot of the final jam thickness profiles for cases of relatively small, medium, and large ice momentum effects $\left(\bar{\eta} / \eta_{\text {eq }}\right)$. A smaller ice momentum effect results in a significantly more uniform thickness profile.

Table 3. Characteristics of various inflow hydrographs.

\begin{tabular}{ccccc} 
Hydrograph type & Initial $\mathrm{Q}\left(\mathrm{m}^{3} / \mathrm{s}\right)$ & Final $\mathrm{Q}\left(\mathrm{m}^{3} / \mathrm{s}\right)$ & $\Delta \mathrm{Q}\left(\mathrm{m}^{3} / \mathrm{s}\right)$ & $\Delta \mathrm{Q} / \mathrm{Q}$ \\
\hline 1 & 100 & 112.5 & 12.5 & 0.125 \\
2 & 100 & 125 & 25 & 0.25 \\
3 & 100 & 150 & 50 & 0.50 \\
4 & 100 & 175 & 75 & 0.75 \\
5 & 100 & 200 & 100 & 1.00 \\
6 & 100 & 250 & 150 & 1.50 \\
7 & 100 & 300 & 200 & 2.00 \\
8 & 112.5 & 200 & 87.5 & 0.78 \\
9 & 125 & 200 & 75 & 0.60 \\
10 & 150 & 250 & 100 & 0.67 \\
\hline
\end{tabular}




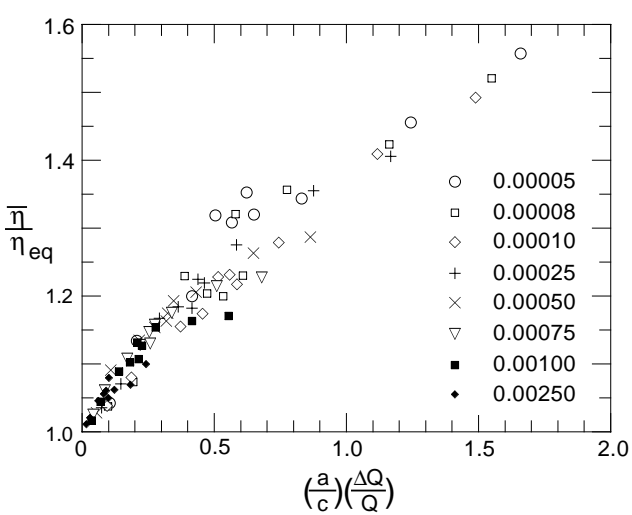

Figure 58. Dimensionless jam thickness vs. ice momentum parameter for several bed slopes.

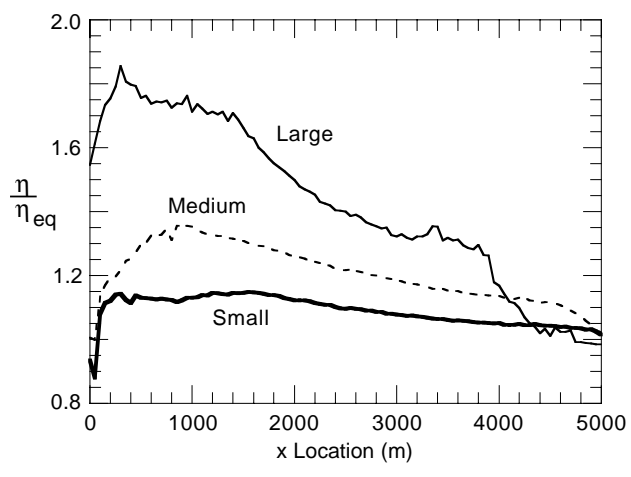

Figure 59. Final jam thickness profiles for large, medium, and small ice momentum effects.

A curve such as the one presented in Figure 58 could be used to determine the general effects of ice momentum on predicted changes in water discharge. A few simple calculations would allow a determination of whether a static, steady flow model is satisfactory or if a fully coupled model such as the one presented here is required to provide accurate jam thickness, and thus water surface, profiles.

\section{Effects of hydrograph shape on jam thickening}

The experiments discussed in the previous section show that the relative state of initial stability of a jam, in conjunction with the relative change in water discharge expected, determines whether ice momentum significantly affects jam thickness profile. Other factors may also determine when ice momentum is significant. Field observations have shown, in general terms, that fast rises in discharge because of heavy rain on frozen ground often result in severe ice jams producing devastating flooding. Commensurably, gradual warming trends with slower discharge increases and higher air temperatures, which cause the jam strength to weaken, often result in less dynamic or damaging jams. The experiments discussed in the Laboratory Experiments section attempted to simulate short duration peak flow events by increasing the water discharge and then decreasing it again after a short period. The effect of these short duration peak flows was to stall the progression of the shoving and thickening, resulting in only partially thickened jams. For those cases, the ice momentum may be important, but the peak discharge may be attenuated greatly by the time the disturbance travels the length of the entire system.

A series of experiments was conducted to assess the effects of the shape of the inflow hydrograph on the resulting jam thickness profile and to establish whether ice momentum continues to be a consideration in these cases. Runs were made with various rates of rise on the rising limb of the hydrograph. The baseline runs had a rate of rise of $2.5 \mathrm{~m}^{3} / \mathrm{s}$ for each time step of 0.5 minutes. For these runs, the time step was held constant, but the boundary condition file describing the inflow hydrograph was modified for rise rates of between 10 and $0.3125 \mathrm{~m}^{3} / \mathrm{s}$ for the same 0.5 -minute time step. This modification resulted in times to peak $t_{\mathrm{p}}$ (time for the hydrograph to increase from 100 to $200 \mathrm{~m}^{3} / \mathrm{s}$ ) of 5, 10, 20, 40, 80, and 160 minutes. The inflow hydrographs are shown in Figure 60. Time to peak $t_{\mathrm{p}}$ was related to the base time $t_{\mathrm{b}}$ defined as the time taken for a disturbance to travel the length of the simulated channel. From eq 152 


$$
t_{\mathrm{b}}=\frac{L_{\mathrm{T}}}{c}=\frac{L_{\mathrm{T}}}{\sqrt{g d}}
$$

where $L_{\mathrm{T}}$ is the total length of the modeled system. Runs were made for the values of $t_{\mathrm{p}}$ given above and three different bed slopes-0.00005, 0.0005, and 0.001 . A functional relationship between the ratio of average final jam thickness to equilibrium thickness $\bar{\eta} / \eta_{\mathrm{eq}}$ and $t_{\mathrm{p}} / t_{\mathrm{b}}$ is evident in Figure 61. As the time to peak $t_{\mathrm{p}}$ increases, the average final jam thickness $\bar{\eta}$ approaches that predicted by equilibrium theory $\eta_{\text {eq }}$. The effects of $t_{\mathrm{p}}$ on the shape of the final jam thickness profile is demonstrated in Figure 62, which shows the final profiles for times to peak of 5, 20 (standard), and $80 \mathrm{~min}$ utes for the bed slope of 0.0005 . As $t_{\mathrm{p}}$ decreases, the changes in water discharge for each time step increase, resulting in larger gradients of the variables in the upstream reaches. The gradient terms (e.g., $\partial \eta / \partial x$ ) are not included in the equilibrium thickness formulation of eq 25 and play an important part in the full ice momentum equation. Also, the large changes in water discharge easily overpower the stability of the jam, resulting in significant ice velocities and ice momentum effects.

Experiments were also conducted to investigate the effects of the sustained time at the peak flow $t_{\mathrm{s}}$. For these runs, the standard inflow hydrograph rate of rise of $2.5 \mathrm{~m}^{3} / \mathrm{s}$ increase per time step was used, but the time at which the water flow was held at $200 \mathrm{~m}^{3} / \mathrm{s}$, before being reduced back to the initial value of $100 \mathrm{~m}^{3} / \mathrm{s}$, was varied. The sustained times simulated were 0 (instantaneous peak), 10, 20, 40, 80, and $\infty$ minutes (no discharge decrease) as shown in the inflow hydrographs depicted in Figure 63. These runs were conducted at the same three bed slopes as above.

Figure 64 shows relationships between the ratio of final average thickness to equilibrium thickness and $t_{\mathrm{s}} / t_{\mathrm{b}}$. For the case of the infinite sustained time, a nominal value of $t_{\mathrm{s}} / t_{\mathrm{b}}$ (beyond which there were no changes in jam thickness) was chosen for plotting purposes. Again, the effect of bed slope is clearly seen. It is significant that, as the value of $t_{\mathrm{s}} / t_{\mathrm{b}}$ reaches 1 , the effects of further increases in the

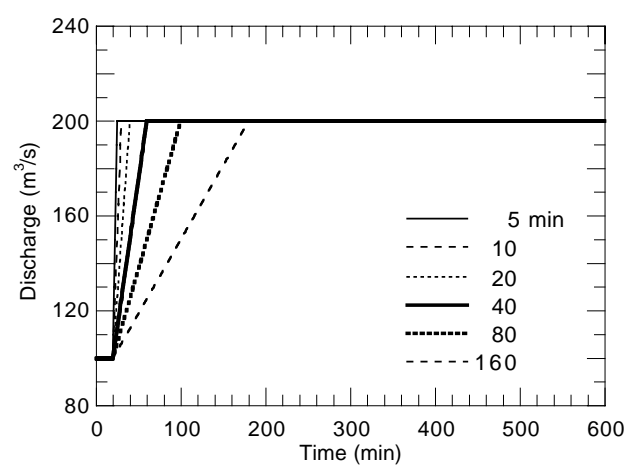

Figure 60. Inflow hydrographs for various times to peak.

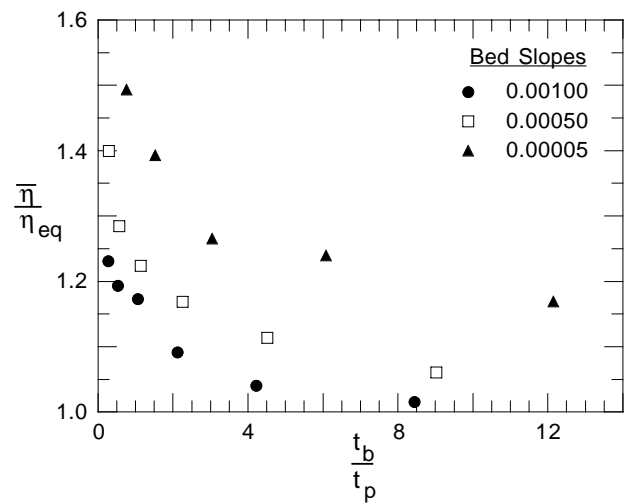

Figure 61. Dimensionless jam thickness vs. $\mathrm{t}_{p} / \mathrm{t}_{b}$ at three bed slopes.

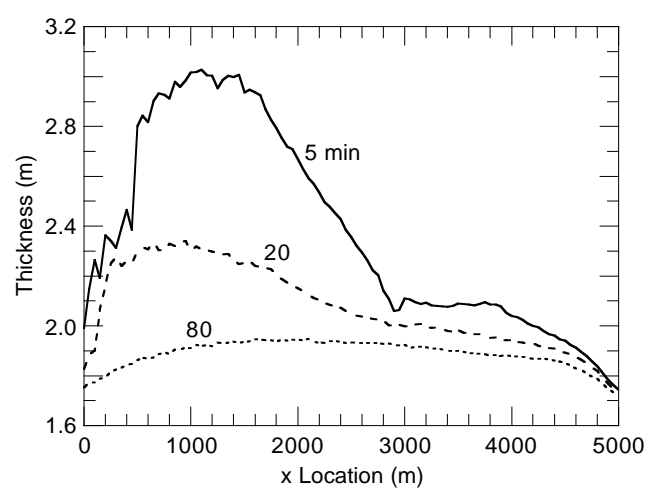

Figure 62. Effect of $\mathrm{t}_{p}$ on final jam thickness profile shape. 


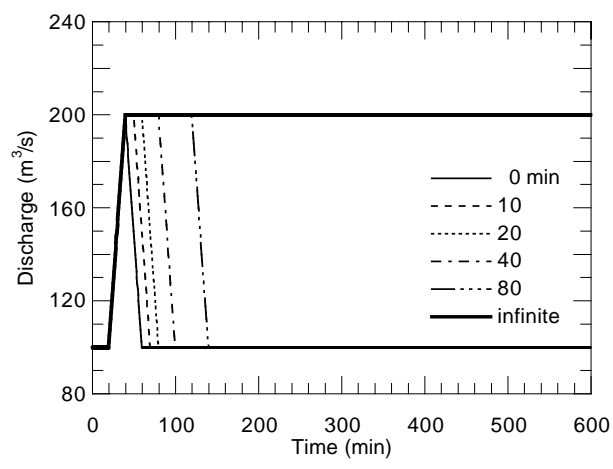

Figure 63. Inflow hydrographs for various times of sustained flow.

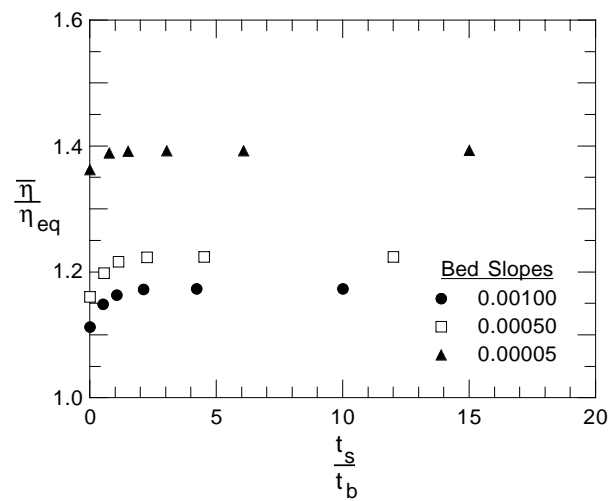

Figure 64. Dimensionless jam thickness vs. $\mathrm{t}_{s} / \mathrm{t}_{b}$ at three bed slopes.

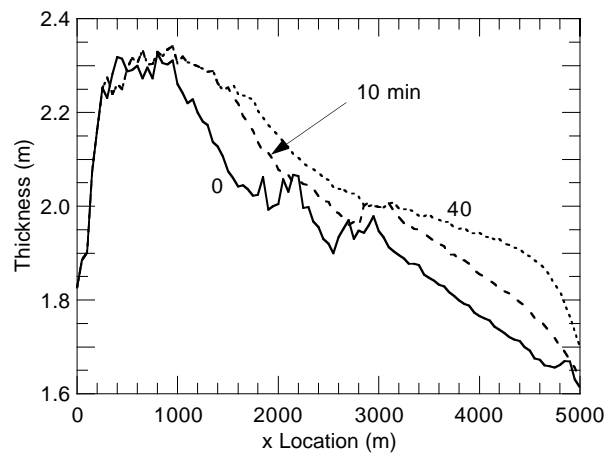

Figure 65. Effect of $t_{s}$ on final jam thickness profile shape. sustained time on the final thickness profile become negligible. The plot does show, though, that even with an instantaneous discharge peak, the effects of ice momentum are important.

Figure 65 presents the changes in the final thickness profile with increased $t_{\mathrm{s}}$ for the 0.0005 bed slope. The increase in $t_{\mathrm{s}}$ results in a slight smoothing and further thickening of the downstream reaches. Even for the instantaneous peak, however, the jam thickness in the upstream reaches is significantly greater than the equilibrium value of $1.7 \mathrm{~m}$.

\section{SUMMARY}

It is well known that ice jams are inherently unsteady events, in which moving ice is brought to rest as accumulations that shove and thicken in accordance with changing forces exerted by water flow, accumulation weight, and bank roughness. These processes are even more unsteady when a jam collapses, plows downstream, and possibly reforms. Not well known, at least in any quantitative way, is how the inherent unsteadiness of those processes affects the jam formation and thickness profile. In particular, virtually nothing is known about the effect of ice momentum on jam formation and thickening. This study presents the first formulation and examination of the fully coupled dynamic nature of the unsteady processes associated with jam formation. It does so by means of numerical simulations and laboratory flume experiments. The experiments also show that equilibrium thickness formulations consistently underestimated measured jam thickness.

The numerical simulation uses the full one-dimensional unsteady flow equations (conservation of mass and momentum) for water flow, ice movement, and jam formation. A unique aspect of the simulation is that the equations are solved in a fully coupled manner. The simulation model is shown to be robust, versatile, and accurate in calculating jam thickness profile under a variety of initial jam, flow, and channel conditions. The effects of the shape of the inflow water hydrograph on jam thickness profile are investigated and shown to be important. A dimensionless pa- 
rameter is used to delineate general conditions for which ice momentum significantly affects jam thickness profile. The parameter takes into account initial ice conditions and the expected flow changes.

\section{CONCLUSIONS}

The study led to the following principal conclusions:

1. The flume experiments, which provide a detailed description of the observed processes of jam formation, failure, and evolution, brought to light two jam failure and reformation mechanisms-progressive and complete. Progressive jam failure and reformation happens with lower initial discharge and lower discharge increases relative to the discharge needed to completely destabilize the jam. It is characterized by a smooth progression of a shoving front downstream through the jam. Complete jam failure, then jam reformation, occurred for initial discharges close to the discharge necessary to completely destabilize the jam. It is characterized by the entire jam mobilizing en masse, moving downstream, slamming into a downstream barrier (e.g., an existing, stationary ice cover, or an ice boom), and reforming.

2. The flume experiments revealed that progressive and complete modes of jam failure and reformation result in measured jam thicknesses that exceeded those predicted using prior jam formulations, based on analyses of stationary jams. This finding confirms that the momentum of the moving ice arrested during jam formation produces an important force that should be taken into account when estimating jam thickness for many conditions. Prior formulations underestimate jam thickness because they do not include this force, or the interaction of the water and ice.

3. The numerical simulation model provides further quantitative information illuminating the effects of ice momentum on jam thickness. Not only does ice momentum result in greater average jam thicknesses than predicted using the stationary jam theory of prior formulations, it also induces a high degree of nonuniformity in a jam thickness profile. The interaction of the ice movement and water flow result in unsteady variations in water depth and velocity, as well as ice thickness and velocity, throughout the entire simulated flow and jam. It is these interactive effects that result in nonuniform jam thickness profiles. For this case of zero ice velocity at the downstream boundary, the profiles are characterized by greater thickness in the upstream reaches where ice velocity and, thus, momentum are greatest. The use of a fully coupled solution technique preserves this interaction of the variables. The loosely coupled solution results in some averaging or smoothing of the variables and their effects upon each other. As the time step decreases or the number of ice calculation cycles increases, the results of the loosely coupled model approach those of the fully coupled model.

4 . The dimensionless parameter $\Omega=(a / c)\left(\Delta Q / Q_{\text {in }}\right)$ is useful for delineating conditions when ice momentum should be taken into account for jam thickness estimation. In $\Omega, a=\left(f_{\mathrm{i}} u^{2} B\right) / 8$, and $c=g s_{\mathrm{i}}(1-p)\left(1-s_{\mathrm{i}}\right) k_{0} \lambda K_{\mathrm{p}} \eta^{2}$, so that $(a / c)$ represents the portion of the initial jam strength mobilized by the water shear stress on its underside. The parameter relates the ratio of average jam thickness, as determined by the fully coupled model, to steady equilibrium thickness determined from stationary jam models. The relationship is useful for establishing when changes in flow conditions (i.e., hydrograph properties) will destabilize a jam and, through ice impact, affect jam thickness profile. The parameter delineates the conditions when a fully 
coupled model of jam evolution is needed to accurately predict jam thickness.

5. The shape of an inflow water hydrograph and its peak flow are important in estimating jam thickness because they determine whether jams fail and reform progressively or completely. Fast-rising hydrographs with attendant rising water levels result in larger gradient terms across a computational reach. The gradient terms (including ice momentum) result in greater predicted jam thickness. They are not present in equilibrium thickness formulations, which thereby underpredict thickness and water levels. The time that a flow remains at its peak value is also important. Instantaneous peaks are attenuated as they travel downstream, resulting in lower local discharges and lower stresses on the underside of the jam, leading to thinner jams.

\section{RECOMMENDATIONS FOR FUTURE RESEARCH}

The flume experiments and numerical model bring to light several areas where deficiencies in knowledge and formulation persist.

Several parameters are necessary to characterize the properties of ice accumulated in a jam. Among the properties are angle of internal resistance $\phi$, coefficient of friction of ice on ice $\lambda$, and the lateral expansion coefficient $k_{0}$. Practical measurements of $\phi$ have been obtained for particulate materials, with an estimate given as the dry angle of repose of the material. No known experiments have been conducted, however, to determine suitable values of $\lambda$ or $k_{0}$ for ice. Further laboratory experiments to define them are necessary.

The full effects of ice momentum have not yet been fully identified because of the influence of ice velocity on the parameters mentioned above. Of particular importance is the temporal variation of these parameters as an ice jam fails and mobilizes, during which the forces on a jam continually change. The value of $K_{\mathrm{p}}$ is related to $\phi$ and has been shown to be adequately described by Mohr-Coulomb theory for a stationary jam. The Rankine states of active and passive pressure described using Mohr-Coulomb theory are intended as point values at the instant of particulate material failure. Questions surround the value of the passive pressure coefficient once a jam fails or is moving downstream. A similar argument goes for the value of coefficient of friction of the ice along the shear boundary at the banks $\lambda$. Certainly, if this coefficient is developed in a way similar to that for the simple static coefficient of friction, there must be changes that occur as the material comes into motion. It is likely that the coefficient would reduce once a jam moves, thereby resulting in a lower resistance to downstream movement. Thicker jams would result when the moving ice is finally arrested because bank friction is reduced.

Several modifications to the model are possible to make the simulations more realistic. The modifications include the ability to use actual cross-section geometry, and changes in roughness coefficients, channel width, and ice parameters with distance. Extending the model to two-dimensional coordinates would not only increase the computational time, but would also introduce difficulties in defining the failure mechanism. The model developed in this study assumes that ice parameters are satisfactorily given as bulk properties. A more suitable advance might be the adaptation of the model to simulate branched systems, where jam formation in one channel would affect water and ice movement in another. 
The very local problem of jam failure also is a candidate for future research. The vertical stress level throughout the thickness of a jam varies linearly when the jam is at rest. This stress description is the basis for the determination of $K_{p}$ and other jam parameters. When water shear stress exists beneath the jam, however, the stress levels within the jam change. No information exists about these stress levels and whether they have an effect in setting up the initial instability within the jam. The vertical description of jam failure, whether the jam initially fails at the bottom or at the water-surface level, where stress is assumed to be the maximum, does not exist.

The highly dynamic nature of ice jamming precludes direct measurement of thickness and ice velocity during formation, evolution, and failure. Equally difficult to obtain are time-histories of water discharge and depth at several locations within a reach experiencing jamming. A concerted effort needs to be undertaken to obtain a complete set of field data for an ice jamming event, including water depths, water velocities, ice thicknesses, and ice velocities at several locations. Another avenue of model verification and validation would be a detailed physical model study of ice jamming, going beyond the level of the flume experiments described in the Laboratory Experiments section. Such a data set will allow the verification and comparison of unsteady ice models, such as the one presented in this study.

\section{LITERATURE CITED}

Ashton, G.D. (Ed.) (1986) River and Lake Ice Engineering. Littleton, Colorado: Water Resources Publications.

Beltaos, S. (1983) River ice jams: theory, case studies and applications. ASCE Journal of Hydraulic Engineering, 109(10): 1338-1359.

Beltaos, S. (1993) Numerical computation of river ice jams. Canadian Journal of Civil Engineering, 20(1): 88-99.

Beltaos, S. (Ed.) (1995) River Ice Jams. Littleton, Colorado: Water Resources Publications.

Beltaos, S., and J. Wong (1986) Downstream transition of river ice jams. ASCE Journal of Hydraulic Engineering, 112(2): 91-110.

Berdennikov, V. (1964) Dynamic conditions of formation of ice jams on rivers. In Soviet Hydrology, Selected Papers. Washington, DC: American Geophysical Union, p. 101-108.

Cunge, J.A., F.M. Holly, and A. Verwey (1980) Practical Aspects of Computational River Hydraulics. Marshfield, Massachusetts: Pitman Publishing, Inc.

Daly, S.F., and G.D. Ashton (1983) Using the DWOPER routing model to simulate river flows with ice. USA Cold Regions Research and Engineering Laboratory Special Report 83-1.

Flato, G.M. (1987) Calculation of Ice Jam Profiles. M.Sc. Thesis. Alberta, Canada: University of Edmonton (unpublished).

Flato, G.M., and R. Gerard (1986) Calculation of ice jam profiles. In Proceedings, 4th Workshop on Hydraulics of River Ice, Montreal, Quebec, Canada, Paper C-3.

HEC (1979) Analysis of flow in ice covered streams using the computer program HEC-2. Davis, California: U.S. Army Corps of Engineers Hydrologic Engineering Center.

Henderson, F.M., and R. Gerard (1981) Flood waves caused by ice jam formation and failure. In Proceedings of the IAHR International Symposium on Ice, Quebec City, Quebec, vol. 1, p. 277-287. 
IAHR (1986) River ice jams: a state of the art report. In Proceedings of the IAHR Symposium on Ice 1986, Iowa City, Iowa, vol. 3, p. 561-594.

Lal, A.M., and H.T. Shen (1991) Mathematical model for river ice processes. ASCE Journal of Hydraulic Engineering, 117(7): 851-867.

Kennedy, R.J. (1958) Forces involved in pulpwood holding grounds-I. Transverse holding grounds with piers. The Engineering Journal, Engineering Institute of Canada, 41: 58-68.

Moberley, H.J., and W.B. Cameron (1929) When Fur was King. Toronto, Canada: J.M. Dent and Sons Ltd., p. 151.

Nezhikhovskiy, R.A. (1964) Coefficients of roughness of bottom surface on slushice cover. In Soviet Hydrology: Selected Papers, No. 2. Washington, DC: American Geophysical Union, p. 127-150.

Pariset, E., and R. Hausser (1961) Formation and evolution of ice covers on rivers. Transactions of the Engineering Institute of Canada, 5(1): 41-49.

Pariset, E., R. Hausser, and A. Gagnon (1966) Formation of ice covers and ice jams in rivers. ASCE Journal of Hydraulic Engineering, 92(HY6): 1-24.

Shen, H.T., H.H. Shen, and S.M. Tsai (1990) Dynamic transport of river ice. Journal of Hydraulic Research, IAHR, 28(6): 659-671.

Tsai, S.M., H.T. Shen, and H.H. Shen (1988) Dynamic transport of river ice and jam initiation. Potsdam, New York: Dept. of Civil and Environmental Engineering, Clarkson University, Report No. 88-2.

USACE (1994) Ice jam flooding: Causes and possible solutions. Washington, DC: U.S. Army Corps of Engineers, Engineering and Design Pamphlet, EP 1110-2-11.

Uzuner, M.S., and J.F. Kennedy (1976) Theoretical model of river ice jams. ASCE Journal of Hydraulic Engineering, 102( HY9): 1365-1383.

White, K.D. (1991) Determining the intrinsic permeability of frazil ice: Part 1. Laboratory investigations. USA Cold Regions Research and Engineering Laboratory, CRREL Report 91-23.

Wuebben, J.L., and J.J. Gagnon (1995) Ice jam flooding on the Missouri River near Williston, North Dakota. USA Cold Regions Research and Engineering Laboratory, CRREL Report 95-19.

Zufelt, J.E. (1992) Modes of ice cover failure during shoving and thickening. In Proceedings of the 11th IAHR International Symposium on Ice, Banff, Alberta, Canada, vol. 3, p. 1507-1514.

Zufelt, J.E., and W.W. Doe (1986) Upper Delaware River ice control—a case study. Proceedings, ASCE Cold Regions Engineering Specialty Conference, Anchorage, Alaska, p. $760-770$. 
Public reporting burden for this collection of information is estimated to average 1 hour per response, including the time for reviewing instructions, searching existing data sources, gathering and maintaining the data needed, and completing and reviewing the collection of information. Send comments regarding this burden estimate or any other aspect of this collection of information, including suggestion for reducing this burden, to Washington Headquarters Services, Directorate for Information Operations and Reports, 1215 Jefferson Davis Highway, Suite 1204, Arlington, VA 22202-4302, and to the Office of Management and Budget, Paperwork Reduction Project (0704-0188), Washington, DC 20503.

\begin{tabular}{|l|l|l}
\hline 1. AGENCY USE ONLY (Leave blank) & $\begin{array}{c}\text { 2. REPORT DATE } \\
\text { December } 1997\end{array}$ & 3. REPORT TYPE AND DATES COVERED
\end{tabular}

\begin{tabular}{|l|l|l} 
4. TITLE AND SUBTITLE & 5. FUNDING NUMBERS
\end{tabular}

Unsteady Ice Jam Processes

6. AUTHORS

Jon E. Zufelt and Robert Ettema

7. PERFORMING ORGANIZATION NAME(S) AND ADDRESS(ES)

8. PERFORMING ORGANIZATION

REPORT NUMBER

U.S. Army Cold Regions Research and Engineering Laboratory

72 Lyme Road

Hanover, New Hampshire 03755-1290

9. SPONSORING/MONITORING AGENCY NAME(S) AND ADDRESS(ES)

10. SPONSORING/MONITORING

AGENCY REPORT NUMBER

Office of the Chief of Engineers

Washington, DC 20591

11. SUPPLEMENTARY NOTES

For conversion of SI units to non-SI units of measurement, consult ASTM Standard E380-93, Standard Practice for Use of the International System of Units, published by the American Society for Testing and Materials, 1916 Race St., Philadelphia, Pa. 19103.

12a. DISTRIBUTION/AVAILABILITY STATEMENT

Approved for public release; distribution is unlimited.

Available from NTIS, Springfield, Virginia 22161

12b. DISTRIBUTION CODE

13. ABSTRACT (Maximum 200 words)

Ice jams cause flooding in northern temperate-climate areas, usually forming rapidly, often with little warning, constricting water flow and elevating water levels. Consequently, jam formation comprises highly unsteady processes: drifting ice pieces are brought to rest, accumulated ice shoves and thickens, and initial water depths and velocities change. Those processes are even more unsteady when a jam collapses. Prior simulations of ice jams, however, treat them as simply stationary, uniformly thick accumulations of ice pieces. No account is taken of the impact forces exerted by moving ice, an estimation that is further complicated by the need to couple equations describing water flow and ice movement. Under the dynamic conditions attendant to jam formation, water flow and ice movement interactively influence each other. This report evaluates the importance of ice momentum on ice jam thickness and thickness distribution using experiments conducted with laboratory flumes and a numerical model in which the equations of motion for one-dimensional flow of water and ice are solved as fully coupled. In this regard, the model is unique, enabling simulation of the important unsteady interactions of water and ice, and determination of their effects on jam thickness. Ice momentum should be taken into account for most jams because it leads to significantly thicker jams and affects the thickness profile. A useful dimensionless parameter is identified for generalizing this finding.

\begin{tabular}{|c|c|c|}
\hline \multicolumn{2}{|c|}{$\begin{array}{l}\text { Ice jams } \\
\text { Laboratory experiments }\end{array}$} & $\begin{array}{l}\text { Simulations } \\
\text { Unsteady processes }\end{array}$ \\
\hline $\begin{array}{l}\text { 17. SECURITY CLASSIFICATION } \\
\text { OF REPORT }\end{array}$ & $\begin{array}{l}\text { 18. SECURITY CLASSIFICATION } \\
\text { OF THIS PAGE }\end{array}$ & $\begin{array}{l}\text { 19. SECURITY CLASSIFICATION } \\
\text { OF ABSTRACT }\end{array}$ \\
\hline UNCLASSIFIED & UNCLASSIFIED & UNCLASSIFIED \\
\hline
\end{tabular}

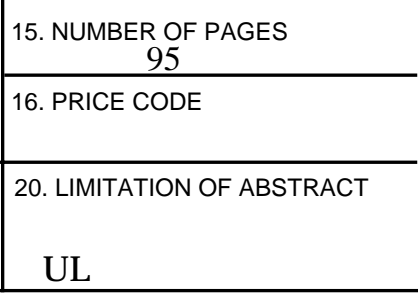

